\title{
Providing Technology Information, Products, and Services (TIPS) Through the World Wide Web
}

\author{
E. William East, Sara E. Ort, and William D. Goran
}

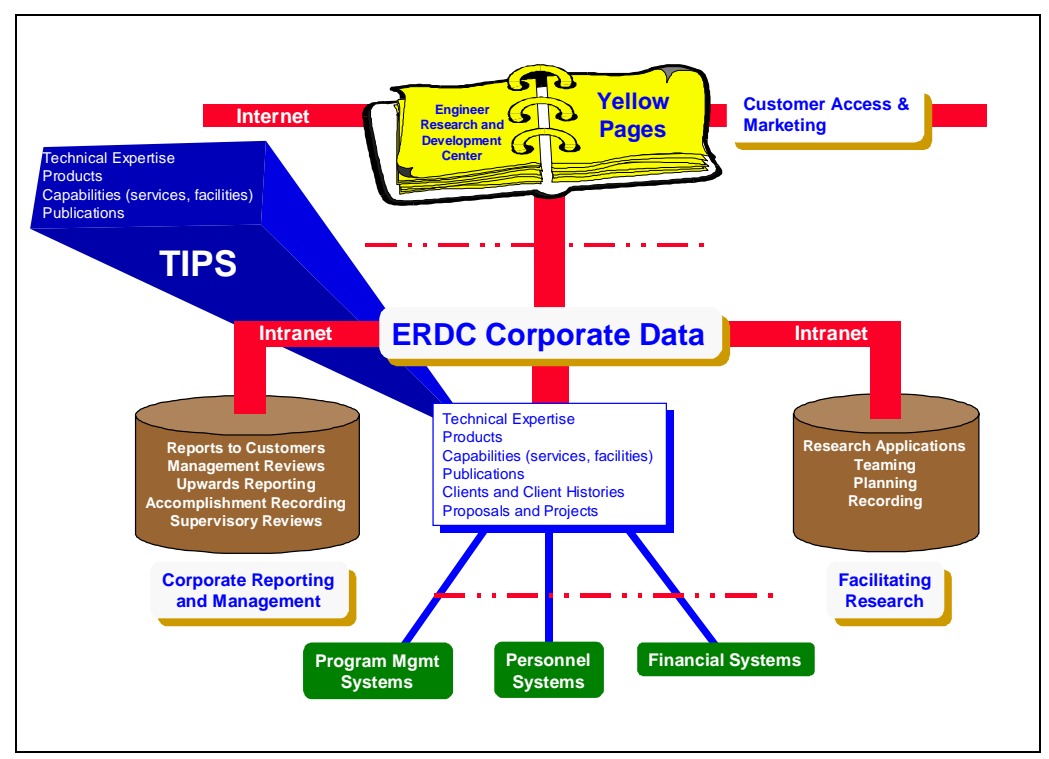

The Technology Information, Products, and Services (TIPS) system is an effective tool for the capture and transfer of information about the CERL campus of the Engineer Research and Development Center (ERDC) assets. In TIPS these assets include people, capabilities, products, and publications.

Relevant information is captured as part of the daily business practices of ERDC researchers, supervisors, managers, and staff. Once approved, appropriate information is provided to potential internal and external ERDC customers.
This work builds upon the prototype Conservation Technology web site. Based on technical support feedback and a formal survey of users of the initial prototype, requirements for the TIPS were developed.

This report documents the system design, business processes, and database structures of TIPS. Initial requirements for implementation of TIPS at all ERDC campuses are also addressed. Draft Corps of Engineers' Life-Cycle Management Information System (LCMIS) documentation was prepared to document system requirements. 


\section{Foreword}

This project was conducted for the U.S. Army Corps of Engineers, Engineer Research and Development Center (ERDC) under project "Generic Business Area Website." The technical monitor was J eff Walaszek (CV-PP).

The authors would like to acknowledge the contribution of the following individuals (listed alphabetically) without whom this project could not have been accomplished:

Douglas Ellsworth (CECER-TD)

Dana Finney (CEERD-PA-I)

Tom Hart (CERD-M)

Gregg Hoge (CEERD-IM-H)

David J ohnson (CECER-CN-B)

Robert Lacey (CECER-TD)

J erome Mahloch (CEWES-ZT-S)

Richard Marlatt (CECER-TD)

Matthew Richards (CEERD-IM-IS)

Robert Riggins (CECER-CN-C)

Richard Schneider (CECER-CN-E)

Charles Schroeder (CECER-CN-B)

J effrey Wal aszek (CV-PP)

The work was performed by the Engineering Processes Branch (CF-N) of the Facilities Division (CF), U.S. Army Construction Engineering Research Laboratory (CERL). The principal investigator was E. William East. Dr. Michael P. Case is Chief, CF-N, and Michael Golish is Chief, CF. The technical editor was Linda L. Wheatley, Information Technology Laboratory.

The Director of CERL is Dr. Michael J . O'Connor. 


\section{Contents}

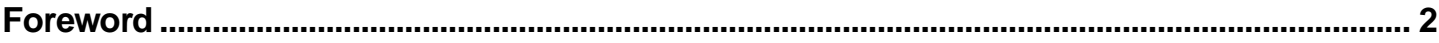

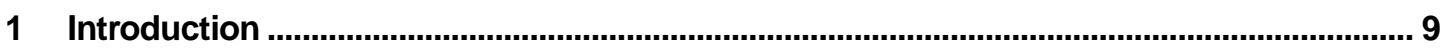

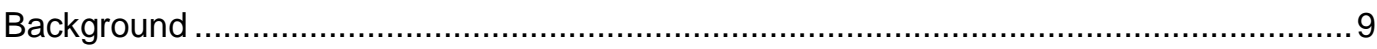

Objectives

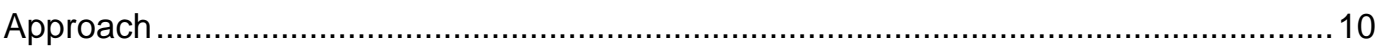

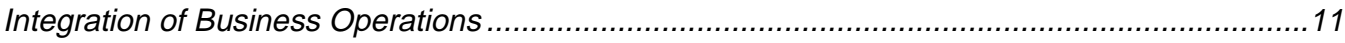

Ease of Customer Use .................................................................................................12

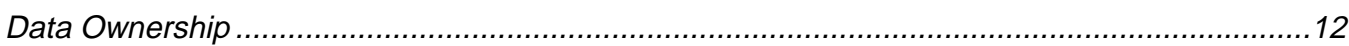

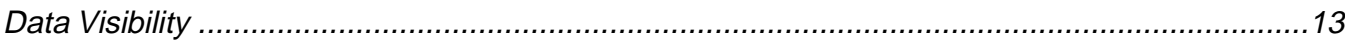

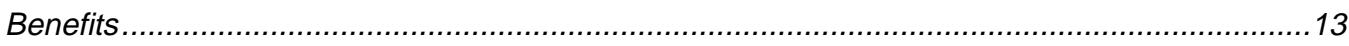

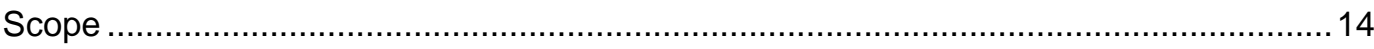

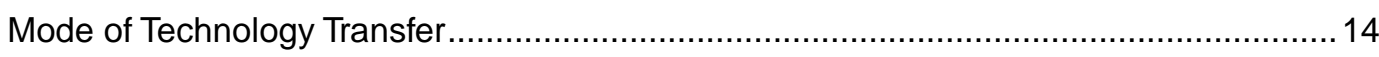

2 Overall System Features.................................................................................................... 15

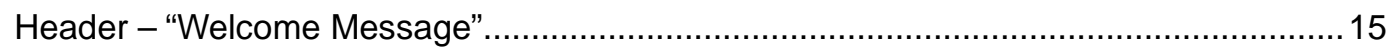

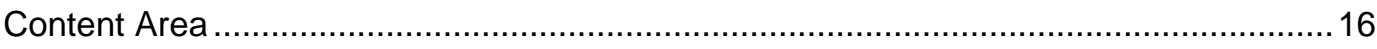

Content Area - "Business Area Description"................................................................... 17

Content Area - "What's New" and "What's Hot" ...........................................................17

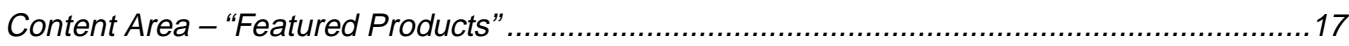

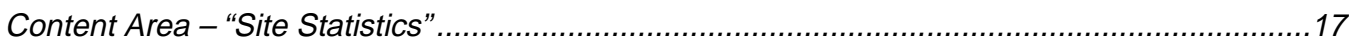

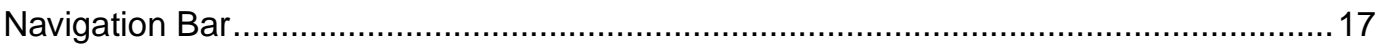

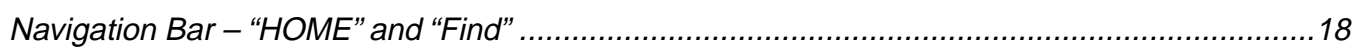

Navigation Bar - "Research Themes" ................................................................... 18

Navigation Bar - "Our Resources" ............................................................................. 18

Navigation Bar - "Join Our Community".................................................................... 19

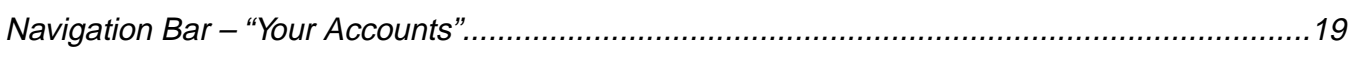

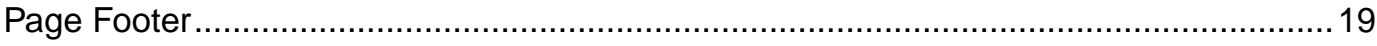

3 User Access Levels............................................................................................ 20

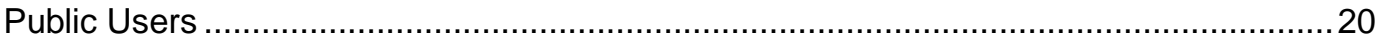

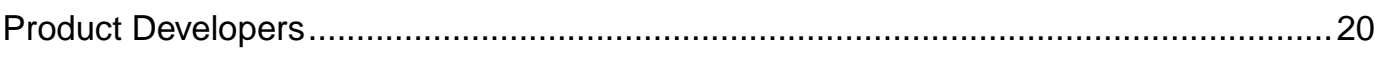

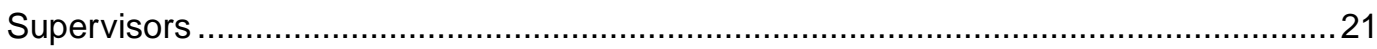

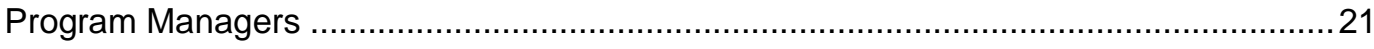

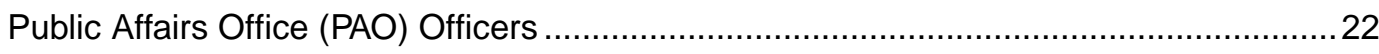

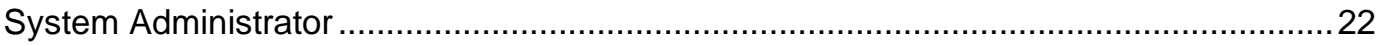




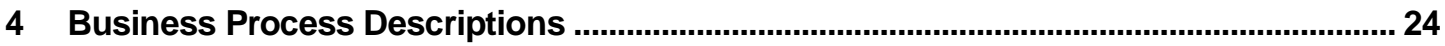

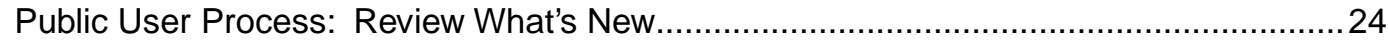

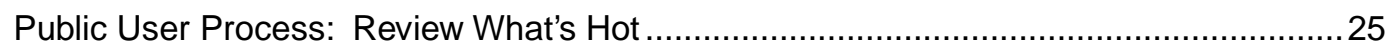

Public User Process: Review Featured Products.....................................................26

Public User Process: Review Research Themes .......................................................27

Public User Process: Find and Contact People ....................................................... 28

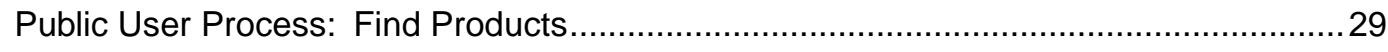

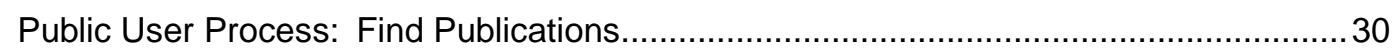

Public User Process: New User Registration ........................................................... 31

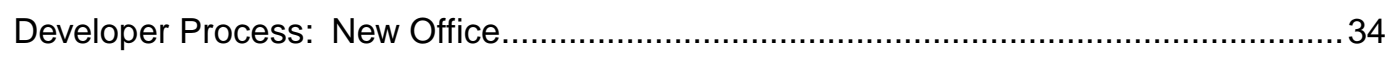

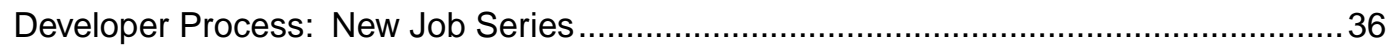

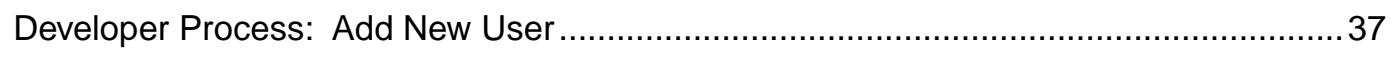

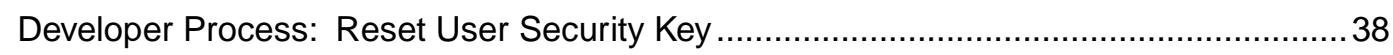

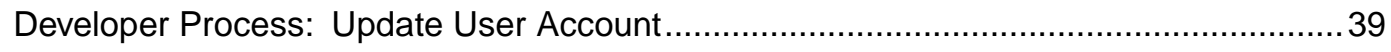

Developer Process: User Resume ......................................................................... 41

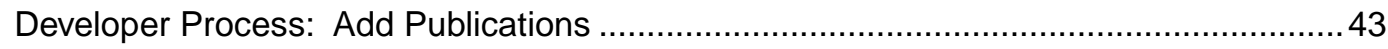

Developer Process: Edit Publications ................................................................... 47

Developer Process: Add Products/Services ........................................................... 48

Supervisor Process: Approve Resumes .............................................................. 49

Supervisor Process: Approve Products …............................................................ 50

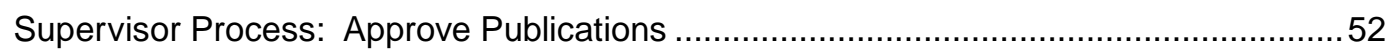

Program Manager Process: Add Research Theme ...................................................... 53

Program Manager Process: Update Research Themes ............................................5 54

Program Manager Process: Approve Research Themes.......................................... 55

Program Manager Process: Designate Hot Products ..................................................56

Program Manager Process: Designate Hot Publications ............................................5 57

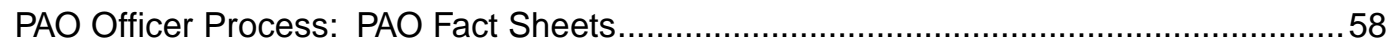

Administrator Process: Update Administrative Settings ............................................. 61

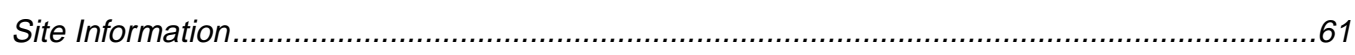

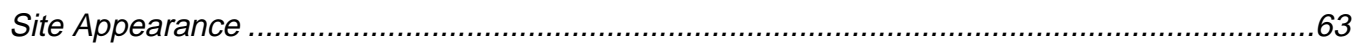

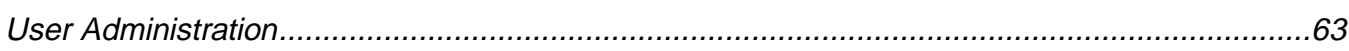

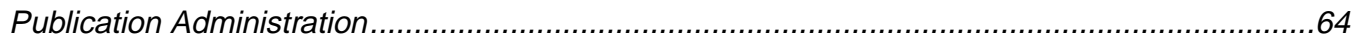

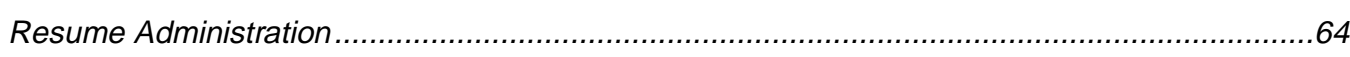

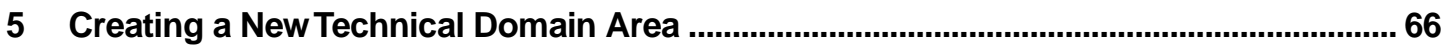

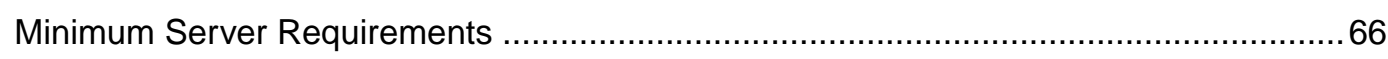

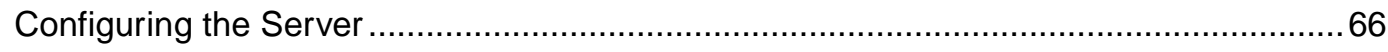

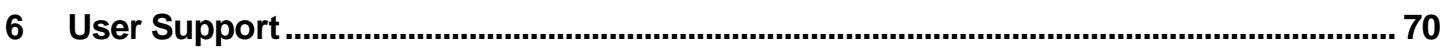

7 Project Management Plan and Evaluation ............................................................................... 72

Phase I and II: Development of an Operational Prototype .......................................... 72 


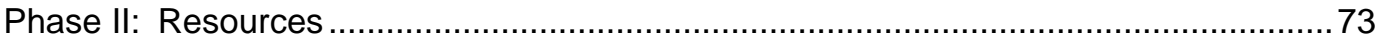

Phase II Evaluation: Opportunities and Challenges.....................................................74

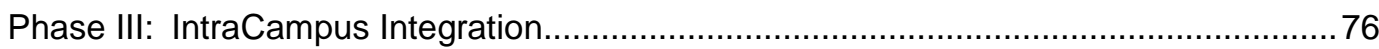

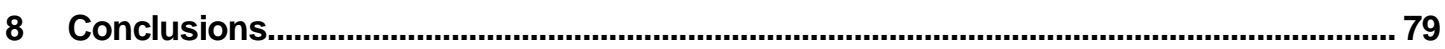

Appendix A: Default Cascading Style Sheet Definitions ..................................................... 81

Appendix B: TIPS Database Structure ...................................................................... 86

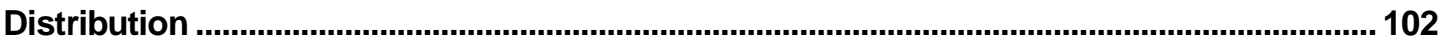

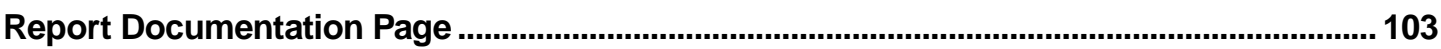




\section{List of Figures and Tables}

\section{Figures}

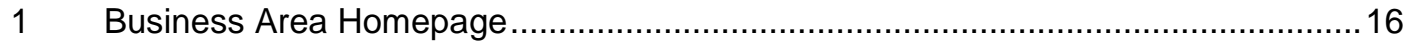

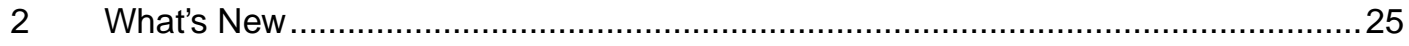

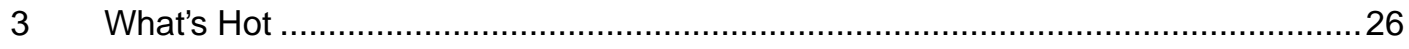

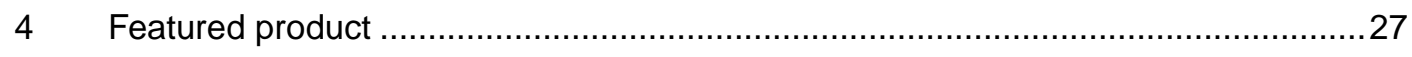

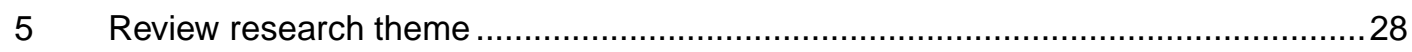

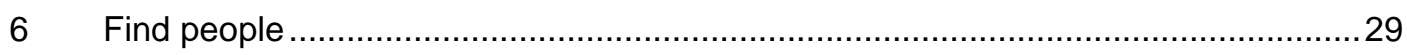

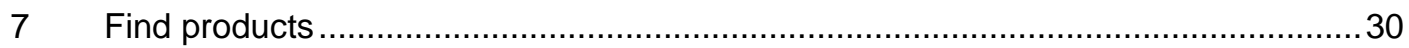

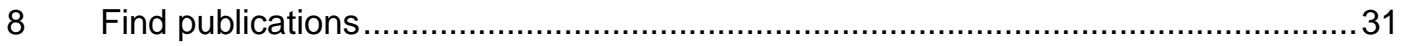

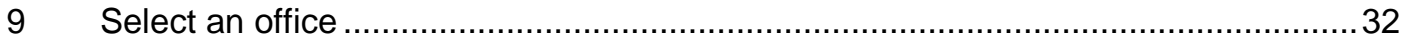

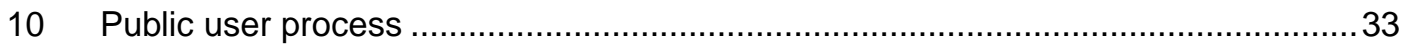

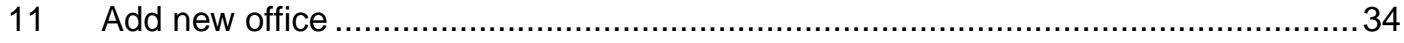

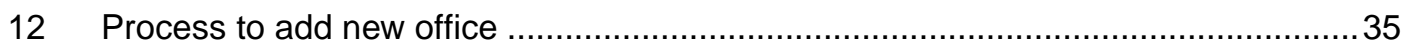

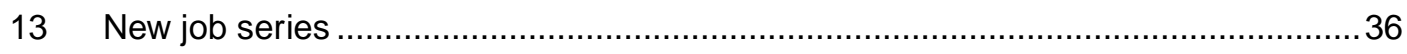

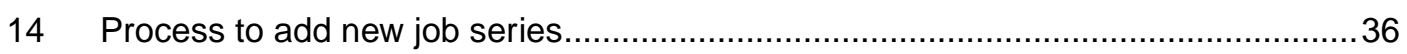

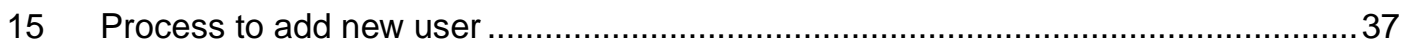

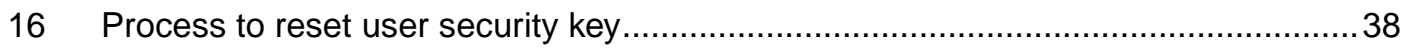

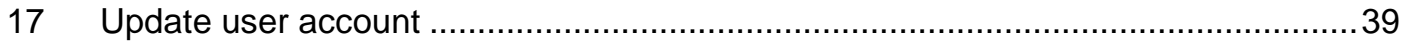

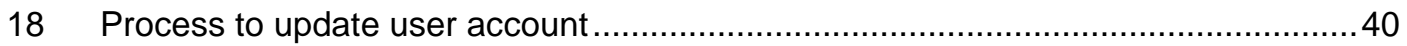

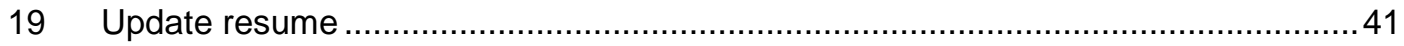

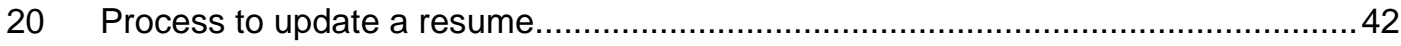

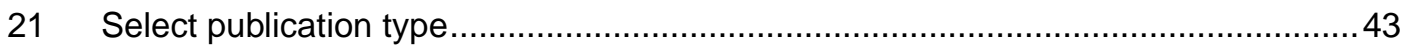

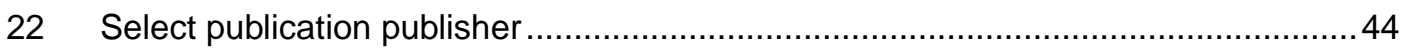

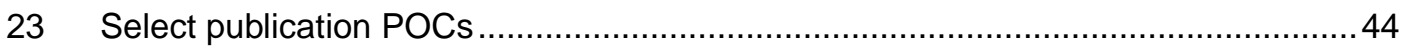

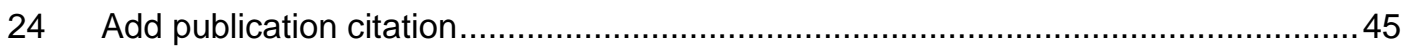

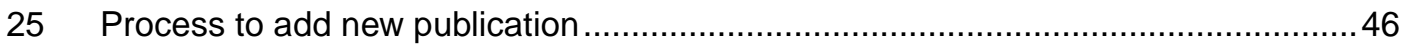

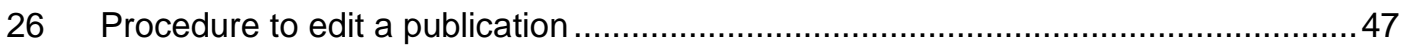

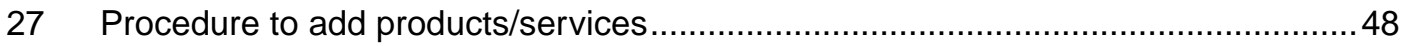

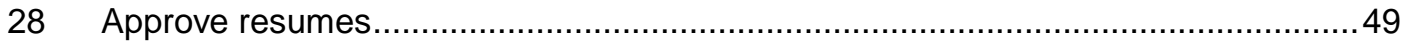

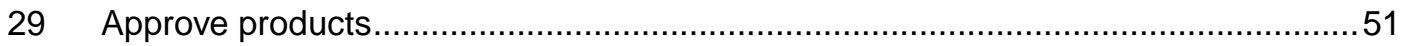




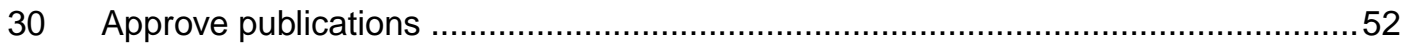

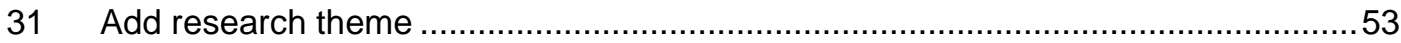

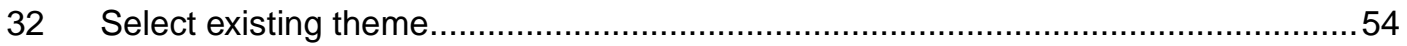

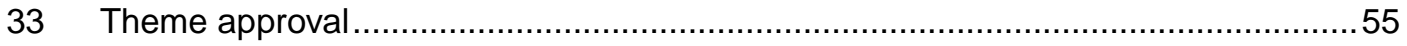

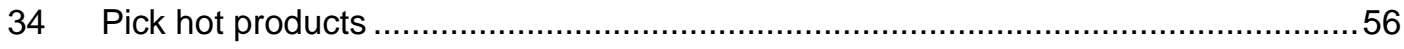

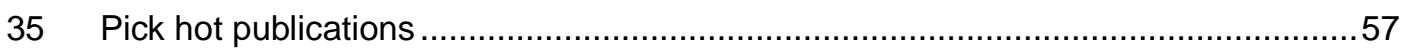

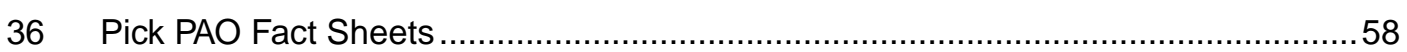

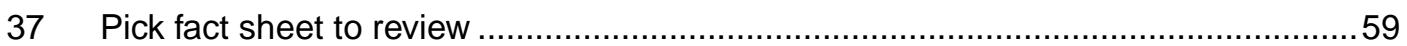

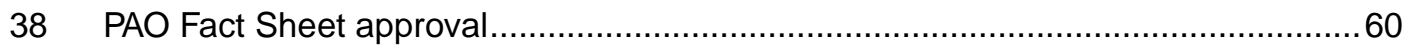

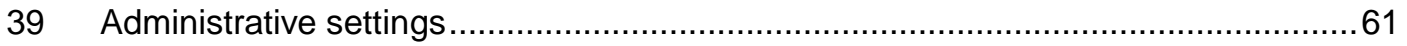

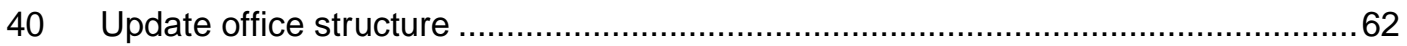

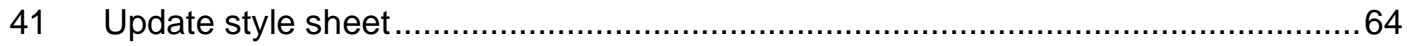

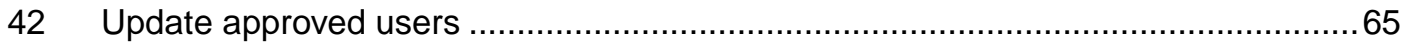

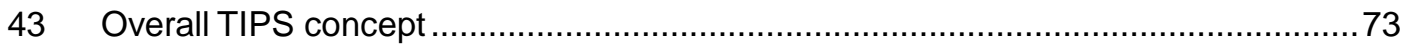

\section{Tables}

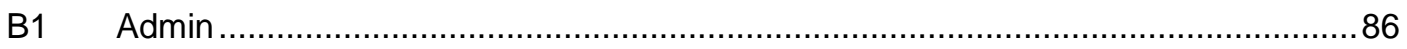

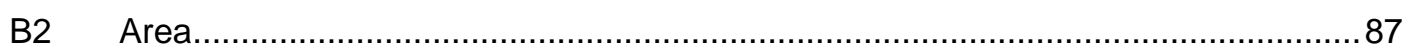

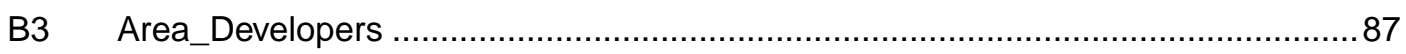

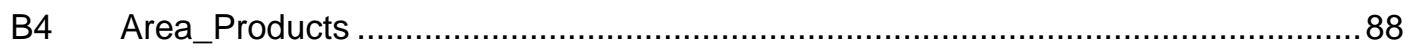

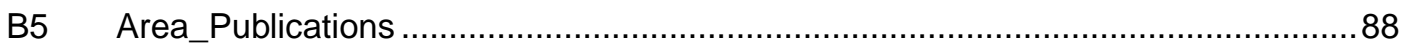

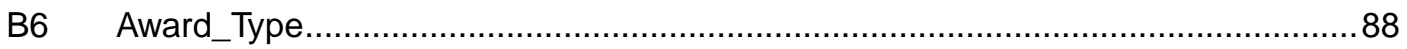

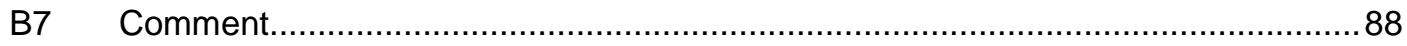

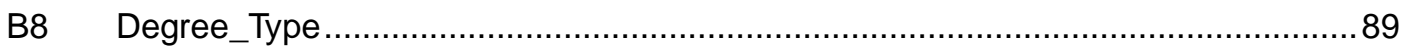

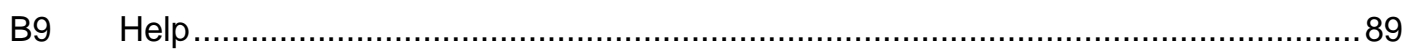

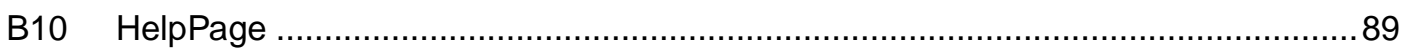

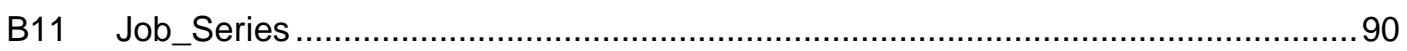

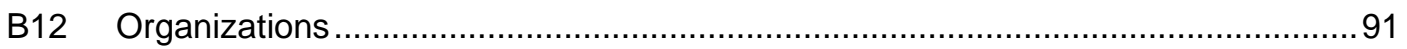

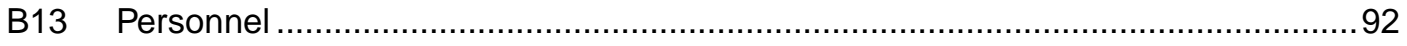

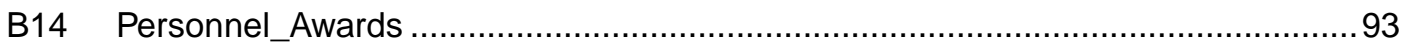

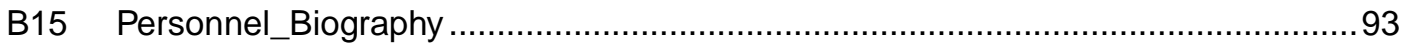

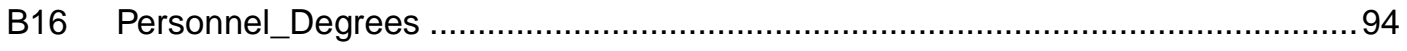

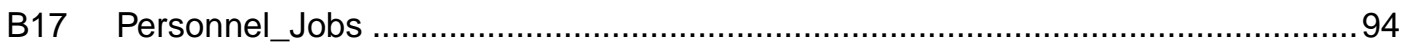

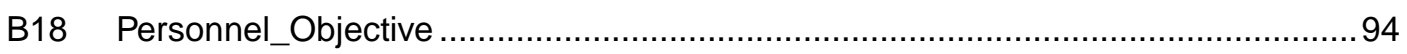

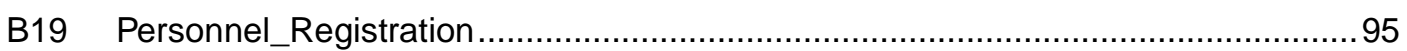

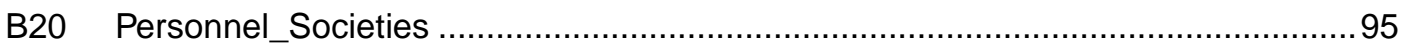




\begin{tabular}{|c|c|}
\hline 21 & Process_Temp \\
\hline 22 & Product_Category.............. \\
\hline 23 & Product_Developers..... \\
\hline & Product_Links ............. \\
\hline 25 & Product_Sponsors ............... \\
\hline & Product_TechTransfer ....... \\
\hline 27 & Products ......................... \\
\hline & Publication_POC...... \\
\hline 29 & Publication_Type ......... \\
\hline 30 & Publications.... \\
\hline 1 & Publishers ................ \\
\hline & Registration_Type... \\
\hline & SocietyStatus ........ \\
\hline & tyle Sheet.. \\
\hline
\end{tabular}




\section{Introduction}

\section{Background}

As the web has emerged as a communication and collaboration media, Construction Engineering Research Laboratory (CERL) has tested various approaches to using the web for capturing information and transferring technology. During 1996 and 1997, an on-line annotated bibliography was developed for all technical publications related to Military Land Management. Then, in 1998, a prototype web site was developed for Military Land Management. This web site, called the "Department of Defense Environmental Conservation Technology Web Site" or "Conservation Technology Web Site," included the on-line bibliography and additional information on related products, capabilities, and appropriate researchers' vitae. The Conservation Technology Web Site began operating in early 1998.

The Conservation Technology Web Site provided the means for users to easily find products related to a technology or service being investigated, validate researcher qualifications through a review of up-to-date researcher job histories, and find answers to questions by searching and downloading on-line publications.

After the usefulness of capturing product information in the Conservation Web Site was demonstrated, the information collected on product status and productspecific technology transfer activities was expanded. This information was used to produce the "Conservation Life Cycle Technology Infusion Plan" (LCTIP) for the Army's Conservation Technology Team.

The LCTIP is intended to improve the process of technology infusion. The plan provides extended "fact sheets" describing the technol ogies, techniques, and tools completed and under development to support Military Land Management. The fact sheets also provide information on demonstration requirements, technology transfer plans, and support requirements. The LCTIP is updated periodically and used as a source for coordination for technology infusion and funds programming within the Army's conservation community.

Development of the FY98 and FY99 LCTIPs and future updates using TIPS provided a means to continually monitor the status of and plans for technologies 
under development. Rather than an additional burden on researchers, saving the up-to-date information in TIPS as needed to support program planning creates LCTIP.

The Conservation Web Site received considerable interest from customers and researchers during the latter half of 1998. In the fall of 1998, CERL management decided to support the upgrade of the Conservation Web Site and develop similar applications for the two other technical domains under its purview: Installation Operations and Facility Acquisition and Revitalization. The work to update the Conservation web site and include the other two research domains began in October 1998 under the "Technology Information Products and Services (TIPS)" designation.

\section{Objectives}

TIPS has three objectives. The first objective is to provide easily navigable and visually interesting information so customers can quickly target expertise, products, and capabilities of interest.

The second objective of TIPS is to provide ERDC researchers and managers with a repository of information that may be used for their own investigations and to reduce the number of data calls and requests for information related to a researcher's products, publications, and resume related information. Once such information is in place, researchers also have a web-presence without having to learn web-based programming.

Finally, TIPS demonstrates that integrated, web-based e-mail and file management tools organized according to standard business processes can effectively replace current paper processes. The production and approval of Public Affairs Office "F act Sheets" is the first paper process to be eliminated by TIPS. TIPS is a tool to help organizations take advantage of emerging information technologies to improve business processes.

\section{Approach}

TIPS began with a concept of improved use of the web for external and internal communications. A committee at CERL explored and reviewed activities at sites across the Corps of Engineers and ERDC. The most similar effort, being conducted by the South Atlantic Division (SAD), was an extension of the Corps' global e-mail directory. SAD's effort added job series and keyword tags to listings 
in the e-mail directory. To ensure compatibility with potential implementation of the SAD initiatives, TIPS includes job series in vitaes. Unlike the SAD keyword tags, the TIPS personnel search tests against researchers' actual employment history. Other information such as education, publication histories, and professional affiliations are also included in TIPS to support the research community.

After the Conservation Technology prototype was developed, an informal survey of representative users was conducted in late 1998. Survey results indicated a need to support marketing and outreach efforts as the primary focus of the site. Concurrent working group meetings also identified or clarified the following requirements:

1. codification and integration of on-line business processes

2. approval of all on-line materials by designated supervisors

3. notification of changes, or changes in status, to appropriate users

4. improvement to the system's graphic design

5. compatibility for potential customers with version 2.0 browsers.

As TIPS developed it became clear that the system was addressing four overall requirements: integration of business operations, ease of customer use, data ownership, and data visibility.

\section{Integration of Business Operations}

The first requirement is that TIPS data should become the primary way for expertise, publication, and product data to flow through the organization. This information should be reviewed, approved, gathered into reports, and presented to customers on-line through a single portal. For TIPS to be successful, it must be the single place where such information is stored, processed, and retrieved.

Authorized individuals throughout the organization input TIPS data. These individuals must be motivated by the results of their data input efforts to sustain these efforts over time. To ensure that value is being obtained from the web site, customer use and issues must be tracked and addressed, and every possible internal use of the data needs to be exploited by the organization. The paper routing and approval processes that are replaced by TIPS need to be fully retired, and the outcomes of these processes need to be achieved through the web site. 


\section{Ease of Customer Use}

The primary value of TIPS is to provide the names of easy-to-find and access research staff who can hel $p$ with the problem at hand. Customers should be able to find answers to questions such as "Who has worked on this problem?" "Have the labs ever done any work on this issue?" "What was the name of that product I saw demonstrated last week?" "Who is the lab point of contact for this type of issue?" "Didn't I see a report a couple of years back on this topic?"

Linking ERDC technical expertise, products, capabilities, and publications will allow customers to be informed consumers when interacting with the laboratories. As informed consumers, customers can verify the number of publications or products in a specific technical area, know who has been active in a field, and know how many experts ERDC has in a field. Providing this information is good customer relations-it gives the customer command of the information needed to keep up to date in a field, to know who to talk to about problems, and to ask for help in an emergency. TIPS will help build trust between the laboratories and ERDC's customers. Few other organizations provide such comprehensive information in a friendly, easy to use context.

Throughout the history of the laboratories, customers have wanted better information about how work performed for others might be useful to them. TIPS gives visibility to all ERDC products (both little and big) and points of contact for more information about them. As a catalog of work at the laboratories, the TIPS web page links to appropriate on-line reports, downloadable software, or other resources related to the product or capability listed in TIPS, give customers the desired ease of access.

\section{Data Ownership}

In TIPS, the researcher(s) and supervisors who performed the research projects are responsible for maintaining their own information about related products. Typically, but not necessarily, the primary point of contact (POC) will be the researcher who entered the data. Every product, service, publication, or related data element has one or more POCs listed. Those persons are responsible for updating and managing their own information.

Allowing the research staff to publicize their own products assures that no data entries fall through the cracks and become outdated. A person is accountable for all the data for which they are the POC, and regular reminders go out to each person (and their supervising/approving official) to review and update their data. I tems that are overdue may be automatically turned off if not regularly updated. 
Individuals do come and go within an organization, but the departure of a POC does not necessarily mean that items listed under that POC are removed. Rather, the supervisor(s) for the project POC must decide to either remove or reassign each of the POC's entries to another POC. Customers (both internal and external) need to be assured that TIPS data are current, and that every item posted on the internet accessible portion of the site is kept up to date.

\section{Data Visibility}

TIPS is designed with three levels of visibility. Initially, data are submitted through the Intranet or internal organizational portion of the web site for review, routing, editing, and approval. Once data is approved, then it becomes visible through the external Internet. This approach is consistent with Corps of Engineers guidance on review of materials posted to web sites.

Once data is posted, it will be "seen" by the search engine, which operates on the TIPS database. To enable external search engines to see TIPS data and to reduce load on TIPS web servers and databases, HTML, PDF, or other needed document formats may be automatically created and posted to any authorized location on the Internet. Such a capability provides the flexibility needed to scale the application from a small set of local tools to an ERDC-wide extranet application.

\section{Benefits}

The benefits of TIPS will be determined by how efficiently and aggressively an organization uses the site. Researchers interacting with customers should consistently point them to TIPS as a resource. When a customer expresses a problem (especially a problem outside the immediate expertise of the researcher), the researcher has an opportunity to guide the customer through a TIPS query session. Once exposed to TIPS, customers will return to the site, and many web explorers will find TIPS on their own. To keep customers returning to the site, TIPS must be kept current.

Besides the obvious marketing and problem scoping uses for TIPS, there are many potential internal functions. Organizations keep track of achievements, but normally collect information from diverse sources in belabored data calls. TIPS should be the source for these data, and should provide sufficient data for analysis of achievements sorted by organizational units, periods of time, technical domains, etc. 
By converting review/approval/routing processes to the web, TIPS helps organizations eliminate paperwork. This can speed up logjams and enhance results, as sequential paper processes with attached "sticky notes" can become simultaneous and interactive on the web. Web-based business processes do not require submitters, reviewers, approvers to be in their offices. These functions can be performed with the appropriate passwords wherever the web can be accessed.

TIPS also provides important benefits for researchers. Besides giving each researcher (or research team) a ready venue to market their expertise and capabilities, TIPS also provides the information necessary for a large and geographically dispersed organization to nurture broad organizational awareness and virtual teaming. Researchers can use TIPS to browse for peers they might want to ask to participate in an upcoming project, or identify expertise needed to provide consulting advice on a project by reviewing resumes and publications listed in TIPS.

\section{Scope}

The system described in this report is applicable to any organization that would like to share information about people, products, capabilities, and publications. Currently TIPS is being implemented at a single ERDC site for three technical domains. Fiscal Year (FY)00 activities are planned to support multi-campus implementations of TIPS across ERDC and at other sites.

\section{Mode of Technology Transfer}

ERDC is evaluating the use of TIPS to support activities throughout ERDC. At CERL, local regulations or standard operating procedures need to be updated as a result of the new technology provided by TIPS. CERL Regulations (CRs) include CR 25-3, USACERL Publications: Definitions and Approval Requirements. 


\section{Overall System Features}

TIPS features a variety of user interface enhancements over the original "Conservation Web Site." These enhancements begin with a four-part structure that is consistently applied across all TIPS pages. These four parts are Header, Navigation Bar, Page Content Area, and Footer. The Header, Navigation Bar, and Footer contain primarily data-driven content defined by either the program manager or system administrator and by the access level of the user. The page Content Area is also data driven. Registered site users, as part of normal business activities, supply all information provided in the Content Area. After supervisory approval, a non-registered user (i.e., the general public) may view the information.

The following paragraphs describe the Header, Navigation Bar, Page Content Bar, and Footer of the site's homepage. The next section of this report describes the system's features and built-in business processes in detail.

The homepage, Figure 1, provides several key pieces of information to all users when they arrive. First is the name of the technical domain and a way to comment and get help. The name of the technical domain is stored in the TIPS database. This enables site administrators to modify the site names, without using any web programming or HTML coding, as the organization evolves over time.

The "get help" and "information" buttons are easily seen at the top of all of the new site's pages. Clicking on the "get help" button will assist with use of the page. Questions arising from the help button are directed toward technical support staff. The "information" button allows users to interact with the authors of the content of the page. In the case of the homepage, the content authors are those persons identified in TIPS as "Program Managers." Saving customer contacts allows management to review the success of specific products, on-line publications, requests for expert assistance, and other site components.

\section{Header - "Welcome Message"}

The majority of TIPS users should not be those who enter information on products and services, but those in need of such services. Such users who are not 
registered with the site see a wel come message that can be updated by any system administrator with or without any knowledge of web software. A script is also provided to automatically link the site to the "Favorites List" for Internet Explorer users. Registered users entering or approving data receive a personalized wel come message, such as the one shown in Figure 1, noting their names and the date they last visited.

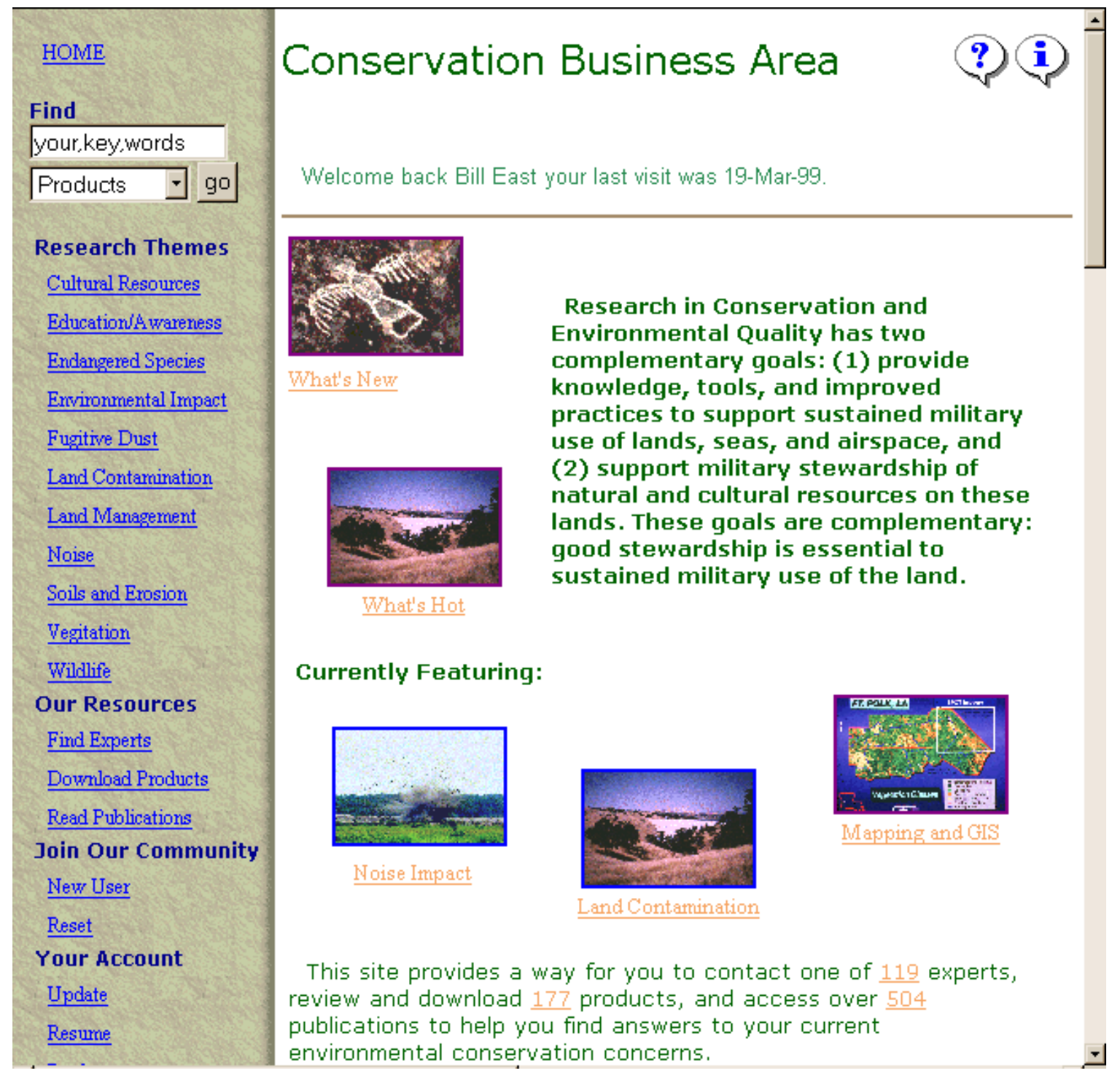

Figure 1. Business Area Homepage.

\section{Content Area}

The next feature of the homepage is the Content Area-just below the welcome message. The center of the Content Area contains four types of information: technical domain description, what products are new or "hot," featured products, and site statistics. 


\section{Content Area - "Business Area Description"}

In the top right of the Content Area (bel ow the horizontal line) is a description of the technical domain. In Figure 1, the description begins with the words "Research in Conservation..." As with all other TIPS content, the data is stored in the TIPS database and may be easily modified by any program manager or site administrator with or without any knowledge of web programming.

\section{Content Area - "What's New" and "What's Hot"}

To the left of the domain description are two graphic links. The first leads to the "What's New" page (Figure 2, p 25), which provides the 10 most recently updated products and publications for the domain. These links are automatically generated. Researchers who want their products and publications to be highlighted on this list will have to keep their information current.

The "What's Hot" page (Figure 3, p 26) shows those products that have been selected by the program manager from the list of approved products and publications. These links may be used to highlight specific products and publications that should be of great interest to the customer community.

\section{Content Area - "Featured Products"}

The third section of the page goes across the center of the page under the "What's Hot" link and the description section. This third section contains three "Featured Products" (Figure 4, p 27). The purpose of these links is to provide direct access to three showcase products in this technical area. Anyone designated as a "Site Administrator" may change the products featured here.

\section{Content Area - "Site Statistics"}

The final section of the Content Area displays the current site statistics and allows users to directly browse people, products, and publications. This section is automatically generated by the system and requires no human intervention. Clicking the links leads users to the respective people, products, or publication browser pages (Figures 6, 7, and 8, pp 29-31).

\section{Navigation Bar}

The left-hand column contains the site Navigation Bar. This list of options includes the home link, quick find, and site menu. The Navigation Bar is partially 
database driven. The page background image (i.e., the brown shading shown in all site figures), research themes, and the number of items under the "Account Info" section are all automatically created from the TIPS database.

\section{Navigation Bar - "HOME” and "Find"}

The HOME link, along with the HOME link in the page footer, allows users to quickly return to the site's main page without clicking the browser's back button repeatedly. The quick find section allows users to find people, products, or publications of interest. The user may enter more than one keyword by separating each word with a comma. The search is then run by clicking the "go" button.

\section{Navigation Bar - "Research Themes"}

Below the search form on the left-hand navigation bar is the technical domain's list of approved research themes pulled from the TIPS database. Program managers or site administrators define the themes. Linked to each theme are a number of graphic files and links to a list of experts who work in that research area (Figure 5, p 28).

\section{Navigation Bar - "Our Resources"}

Below the research themes are links with which to browse people, products, and publications (Figures 6, 7, and 8). These browsing forms show 25 records at a time and begin with the most recently updated records. Clicking one of the four browse buttons allows users to see the first, previous, next, and last set of records. Browse buttons only appear if appropriate to the current location in the record set. For example, if the user is at the beginning of the record set, then the First and Previous browse buttons do not appear.

Using the search form provided on the page reduces the number of records to be browsed. Again only 25 records are shown at a time. If there are more than 25 records in the selected record set, then the browse buttons operate on that selected set until the user resets the query.

Once the user finds a record of interest in either the people, product, or publication browse pages, they may "drill-down" by using the link provided for specific information on the item of interest. Note that these pages are backward compatible and do not require the use of newer browsers. 


\section{Navigation Bar - "Join Our Community"}

The next section of the navigation bar allows new users to register, or existing users to add new users. Users may also reset their accounts, if they change computers, or wish to provide an in-depth demonstration at another computer. A detailed description of the user registration process is provided later in this report.

\section{Navigation Bar - "Your Accounts"}

The final section of the navigation bar, for registered users only, is the user's account section. Only those options for which the user has permissions (based on their system administrator-designated access level) are shown. This section is one method for the user to review, add, or update information to which they have access. There are several levels of access within TIPS. Chapter 3 describes the levels of system access.

\section{Page Footer}

At the bottom of the page, the site footer provides a simplified horizontal navigation bar and information about who to contact in case of problems. The footer information in the Navigation Bar area allows the user to easily return to the top of the current page. Footer information in the page Content Area provides the user an easy way to return to the site Homepage (a database driven link) or to browse people, products, or publications. Site information and POC data including e-mail and telephone numbers are provided at the bottom of the page. The site information, including the homepage URL, company, and POC data, is all obtained from the site database. When the system administrator changes this information, it is updated on all pages. 


\section{User Access Levels}

TIPS has six user access levels. These levels range from unregistered "public users" to "system administrators." These accounts have an increasing ability to approve information for public access and to change system settings. Users may be assigned to multiple access levels. Each type of user receives automatic email notifications containing changes to the data for which they are responsible and the type of task that needs to be performed on that data.

\section{Public Users}

The first access level is for the general public. Public users are identified by the system as not having a site security key. Public users are only able to see approved information about research themes, people, products, and publications.

While public user activity on the web site is not tracked, questions and comments made by the general user community can be saved. Relevant information based on these requests may be supplied automatically to the appropriate developer(s), supervisor(s), program manager(s), or system administrator(s). The value of tracking general counts of public access to project information has yet to be determined.

\section{Product Developers}

Product developers are Principal Investigators and other personnel responsible for research and development. These are the recognized experts to whom the general public (and other researchers) are directed, as needed. As approved by their supervisors, developers may create an on-line resume, maintain publication data, and produce product and service information sheets. Developers may also review system-wide reports. Once a supervisor has enabled the developer to add information to the system, the supervisor must approve the developer's resume, publications, and product information sheets before this data is visible to the general public. 
When a user registers with a given organization, they may be given automated developer permission based on the default permissions of the office under which the user is registering. Otherwise, the system administrator must give new registrants developer status before they can begin adding resume, product, and publication data to the site.

\section{Supervisors}

Supervisors are typically branch chiefs, division chiefs, or their designees who are responsible for traditional activities such as first-line approval of fact sheets, publications, or other promotional materials. Supervisors are responsible for approving developer resumes, products, and publication listings. In addition to approval authority, supervisors are able to contribute their own resume, products, and publications to the site.

As the developers that work for a given supervisor add resumes, publication listings, and product data, the supervisor is sent e-mail notifying them of the changes being made. The e-mail provides a description of what was changed and a link to the data approval page. Approving the changes allows the document to be seen by members of the general public.

To reduce the burden of additional e-mail traffic, it is recommended that supervisors use their e-mail program to direct all TIPS messages to a TIPS activities folder. Such a folder will enable supervisors to review changes to TIPS data on a periodic basis.

\section{Program Managers}

Program managers are responsible for the creation, updating, and approval of research themes and the identification of hot products and publications. In addition to these main responsibilities, program managers have all the access rights of supervisors.

Research themes may be created and edited before being approved for public release. Program managers may assign one or more registered users as the POC for a given research theme. Public users interested in a given theme will then be able to find out more through these POCs. 


\section{Public Affairs Office (PAO) Officers}

A TIPS site is meant to serve as both a collaborative research area and a means of providing information to potential customers. As a result, some of the information contained in the site refers to products and publications that are inprogress or includes links to sites that contain working documents. These working documents are not expected to be of interest to a general audience and should, generally, not be included in a top-level discussion of the technical domain. Since both working documents and successfully completed products that would be of general interest are contained within a TIPS site, a way to identify those documents has been included in the demonstration site.

PAO Officers will be able to designate some of the product materials contained in a TIPS site as official product "fact sheets" or more general "capability description sheets" or "general information sheets." The PAO Officer first identifies a product listing as a potential PAO Fact Sheet. After the PAO Officer reviews the fact sheet, it may be edited for minor changes before being approved for public viewing. Comments by the PAO Officer are kept with the product information for future use and review by developers, supervisors, and program managers.

\section{System Administrator}

The system administrator has access to all of the functions listed in the previous levels and also may revise many of the site settings. These site settings include the site title, name and location of the page, the site wel come message, the site POC footer, and picture files for the "What's New" and "What's Hot" pages.

One of the most important functions of the site administrator is to assign people to one or more roles within the business area web site. The site administrator is the only person who has access to assign users as developers, supervisors, program managers, PAO Officers, or system administrators. Typically the administrator will not, however, need to assign developers, program managers, and PAO Officers since these permissions are assigned based on default user office permissions.

It is recommended that one primary and two backup persons be assigned as system administrators. The best organization is to have people who are also program mangers be the backup system administrators. Multiple assignments allow work to be performed when the primary is unavailable. 
TIPS uses Cascading Style Sheets (CSS) ${ }^{*}$ to format the font, size, color, etc., of the text. Use of CSS allows one centralized set of information to define typefaces, font sizes, font decorations (such as bold or underline), and font color for every page in the demonstration site. This powerful feature allows the system administrator to change the appearance of different business areas. Those areas with specific graphic design themes may be easily implemented. Appendix A contains a list of default CSS information that is currently being used for the TIPS site.

Admi nistrators are also responsible for maintaining the various pick lists used in resumes and publications. These lists include degree types and publishers.

\footnotetext{
* For information on CSS, please see http://www.w3.org/TR/WD-css1.html
} 


\section{Business Process Descriptions}

This chapter describes the processes by which different types of users navigate through TIPS. The purpose of this section of the report is to (1) describe, in detail, the progress of work to date, (2) provide a vehicle for communication about the contents of TIPS, and (3) provide the business process flowcharts that are implemented in TIPS.

Some of the processes included in TIPS are simple; for example, reviewing the latest additions to the site. Other processes, such as changing the office affiliation of a registered user, are not quite as simple. Some processes such as those required to identify and approve official PAO Fact Sheets are meant to replace existing paper-based activities. The business processes currently included in TIPS are not a complete set of integrated business functions, but a jumping-off point for discussion of what an ERDC extranet should ultimately comprise.

\section{Public User Process: Review What's New}

Members of the general public, as well as all registered users, have access to the most recently updated, approved products and publications through links on the "What's New" page, shown in Figure 2. Links take the user directly to the product information or publication detail page. External links may also be followed to external sites, if these links are contained in the approved product or publication data.

The products and publications on the "What's New" page are automatically generated by the system based on the date of the last update for approved products and publications. 


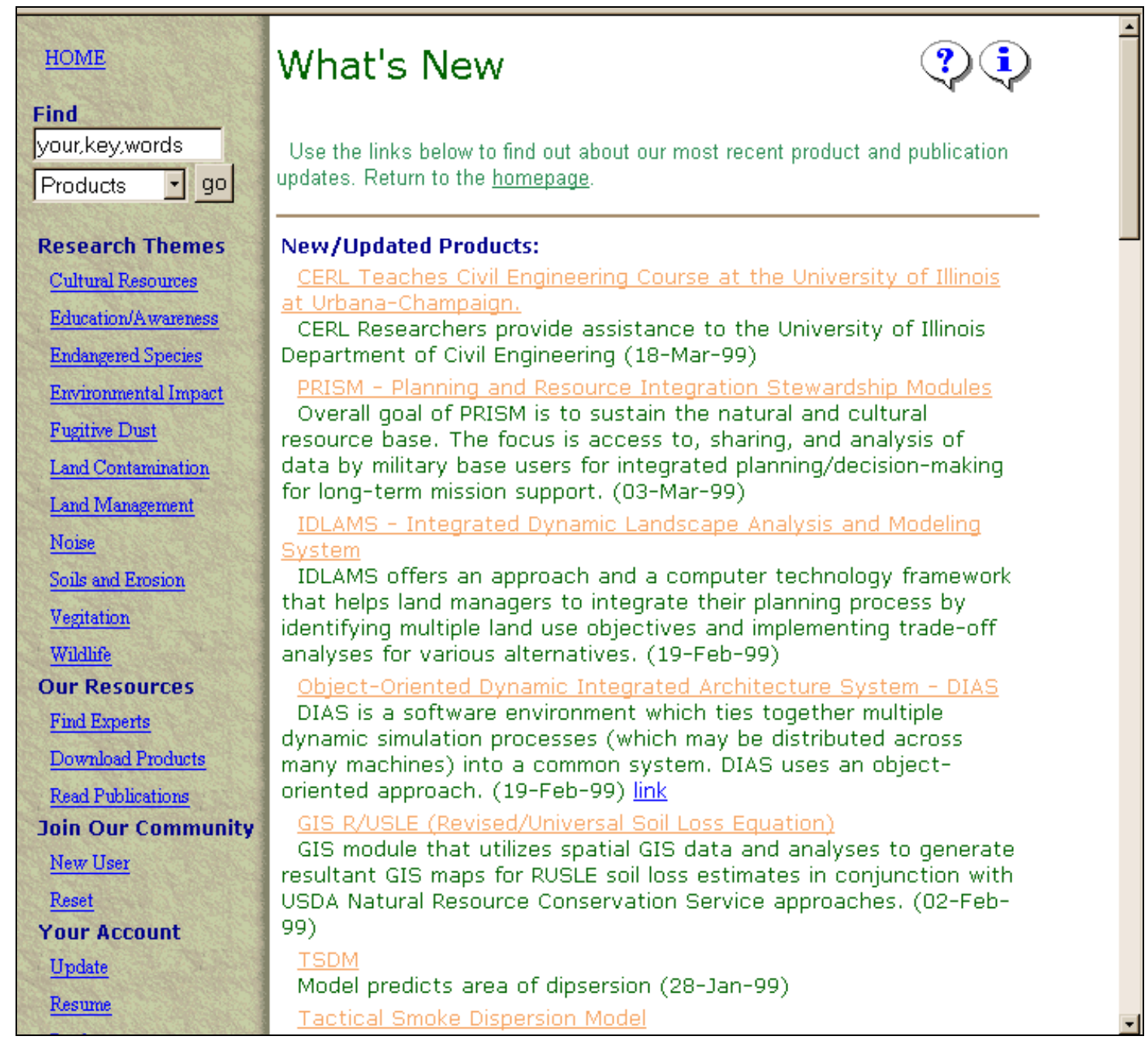

Figure 2. What's New.

\section{Public User Process: Review What's Hot}

Members of the general public, as well as all registered users, have access to the list of "Hot" products and publications through links on the "What's Hot" page, shown in Figure 3. Only program managers or site administrators are able to update hot products and publications as shown in the instructions at the top of the page.

Clicking the item's title takes the user directly to the product or publication detail page. External links may also be followed to external sites, if these links are contained in the approved product or publication data.

The products and publications on the "What's Hot" page are manually linked by the site program manager or site administrator. 


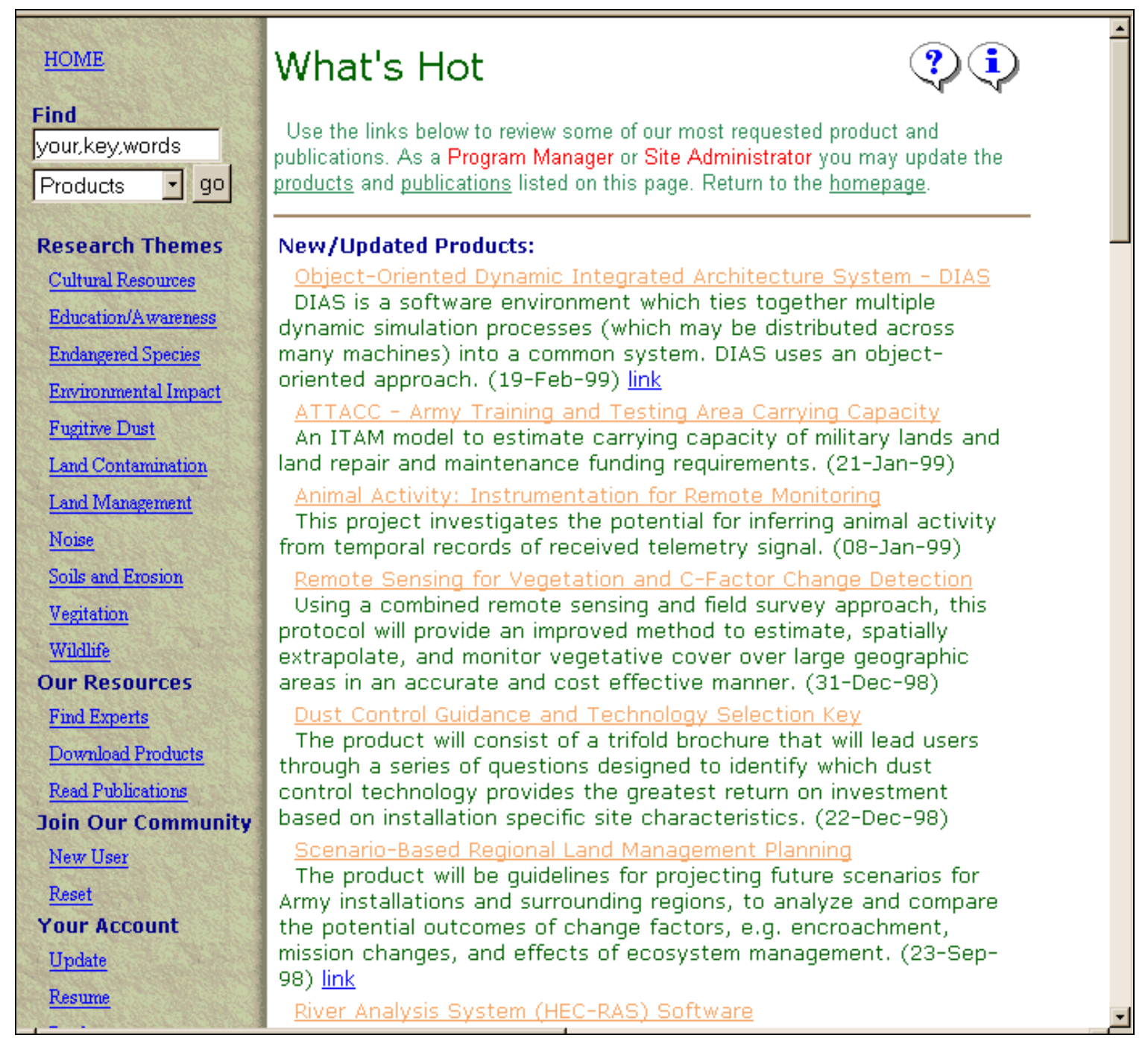

Figure 3. What's Hot.

\section{Public User Process: Review Featured Products}

Members of the general public, as well as all registered users, have access to the three featured product links on the home page. An example of the detailed product listings is shown in Figure 4. As with "Hot Products," only program managers or site administrators can update the featured product links.

Information displayed for the featured product is simply the approved product information sheet for that product. The developer need do no additional work to provide information for a featured product. 


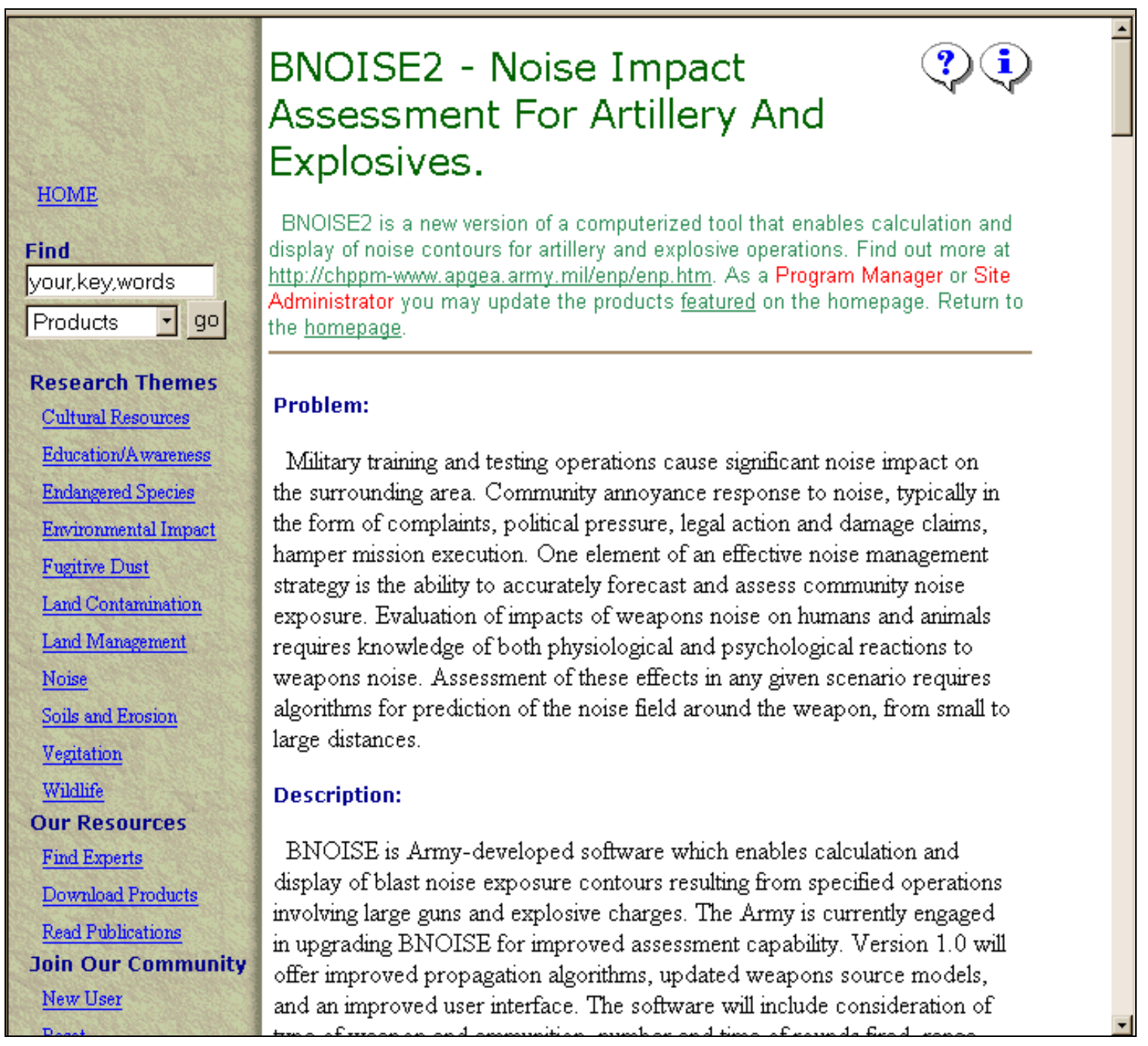

Figure 4. Featured product.

\section{Public User Process: Review Research Themes}

Members of the general public, as well as all registered users, have access to those research themes created and approved by the program manager. Research themes represent the general categories of work conducted in a given technical domain. Figure 5 shows an example of one of the research themes.

The program manager provides the content, graphics, and POCs for the research themes. POCs are listed at the bottom of the research theme description. Research theme descriptions may be used to address "F requently Asked Questions" (FAQs) posed by customers or potential customers of the products under this theme. The technical content of research themes should be limited. 


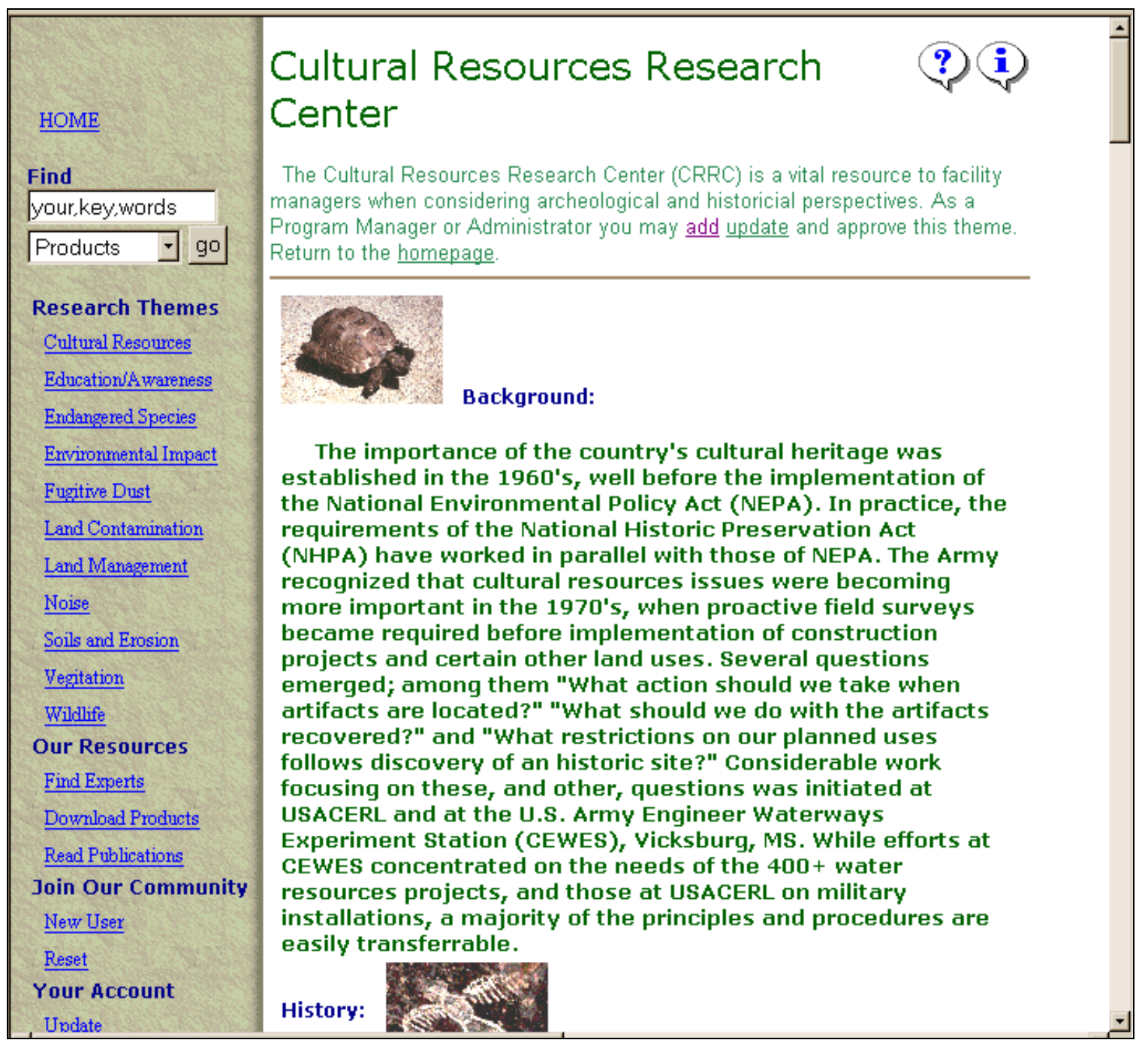

Figure 5. Review research theme.

\section{Public User Process: Find and Contact People}

Members of the general public, as well as all registered users, can find any approved TIPS registrants. Registered TIPS users whose resumes have been approved also have an active resume link. Those members whose resumes have not been approved appear without a link on their name. Figure 6 shows the "Find People" browse and search page.

When a user first opens the page, the first 25 of the most recently updated records appear in the browsing area of the page. The browse buttons allow users to view sets of 25 records at a time. The appropriate browsing buttons appear at the top and bottom of the record set. 


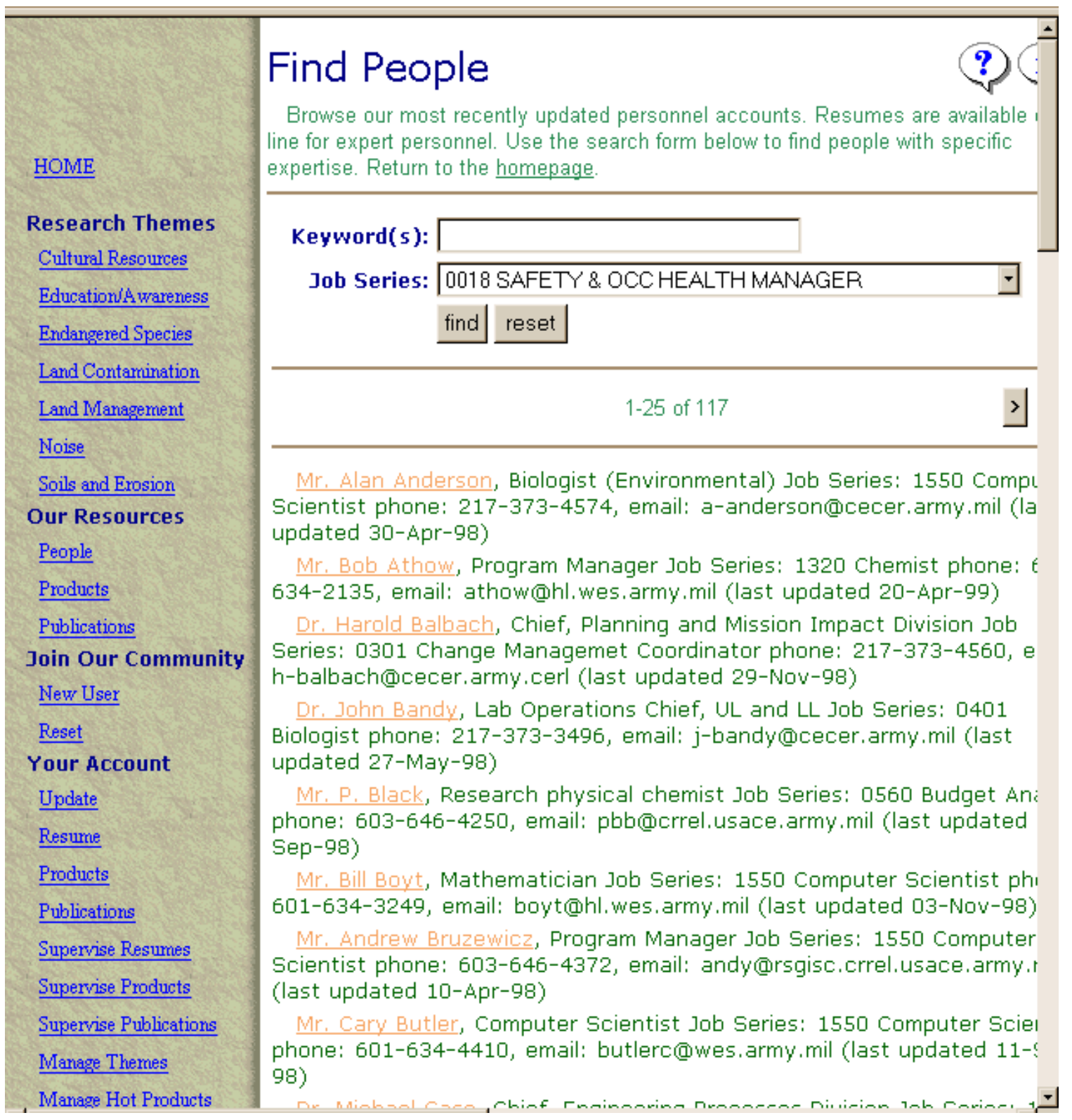

Figure 6. Find people.

Users may search for personnel in the technical areas that have specific types of expertise using the search form at the top of the page. One or more keywords, separated by a comma, may be entered in the form provided to search against each person's job history. Alternatively, users may search for personnel based on their F ederal service job series number.

\section{Public User Process: Find Products}

Members of the general public, as well as all registered users, have access to all approved products contained in the domain. Figure 7 shows the product browse 
and search page. When a user first opens the page, the first 25 of the most re cently updated records appear in the browsing area of the page. The browse buttons allow users to view sets of 25 records at a time. The appropriate browsing buttons appear at the top and bottom of the record set. Users may search for product abstract, problem, technology, or benefit paragraphs using the search form at the top of the page.

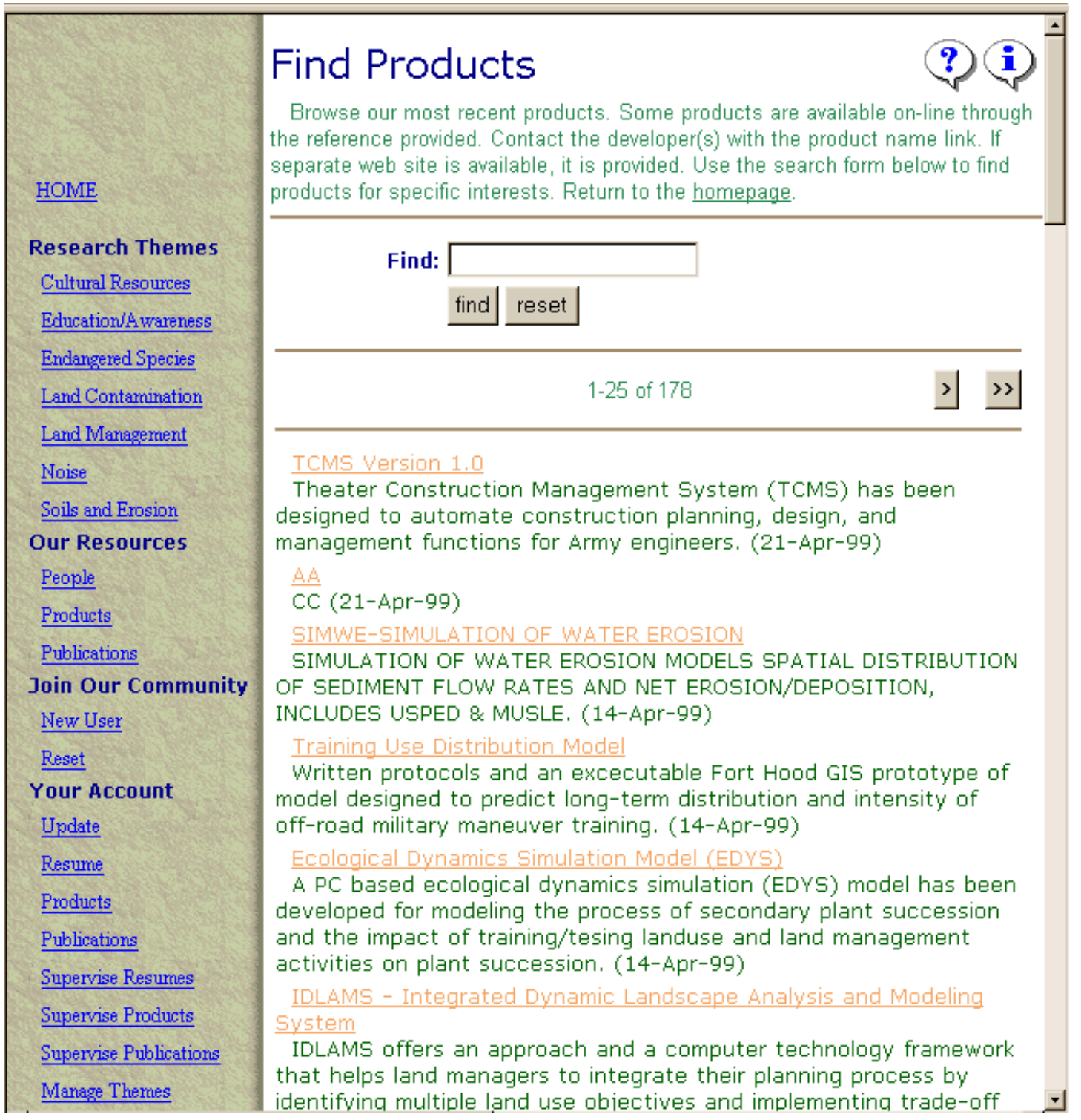

Figure 7. Find products.

\section{Public User Process: Find Publications}

Members of the general public, as well as all registered users, have access to all approved publications contained in the domain. Figure 8 shows the publications browse and search page. When a user first opens the page, the first 25 of the 
most recently updated accounts appear in the browsing area of the page. The browse buttons allow users to view sets of 25 records at a time. The appropriate browsing buttons appear at the top and bottom of the record set. Users may search publication titles and abstracts using the search form at the top of the page.

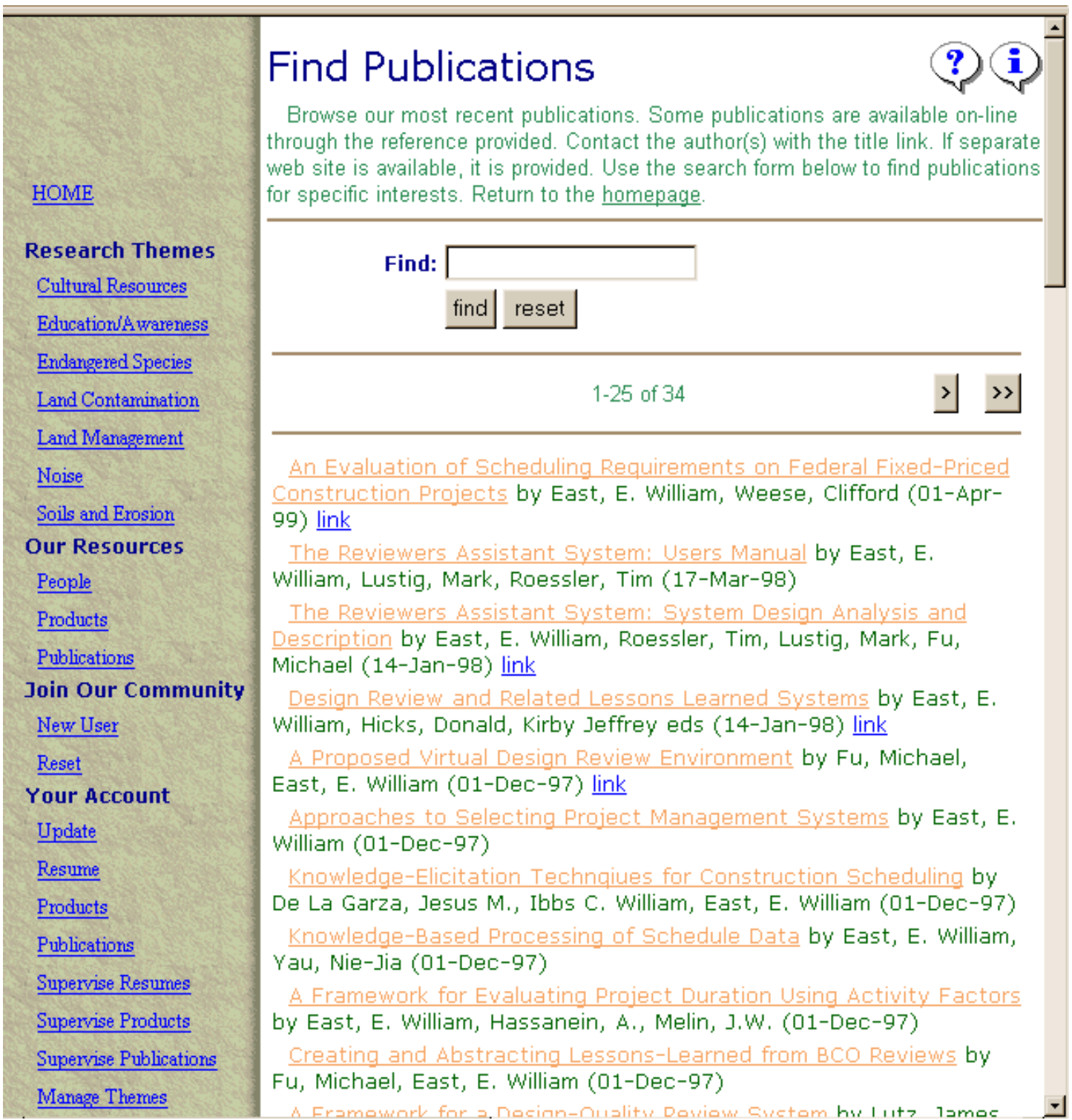

Figure 8. Find publications.

\section{Public User Process: New User Registration}

New user registration occurs in three parts. The first is the identification of the user's office. The office identification is needed to provide an initial security check of the user, and to provide the user's default permissions. 
Part 1 of the registration process is shown in Figure 9. Supervisors must provide office passwords to individual users at a given office before those users register. After the user has provided a valid office/password pair, he/she may proceed to enter personal information. Part 2 of the registration process allows the user to enter his/her name, telephone number, and e-mail address.

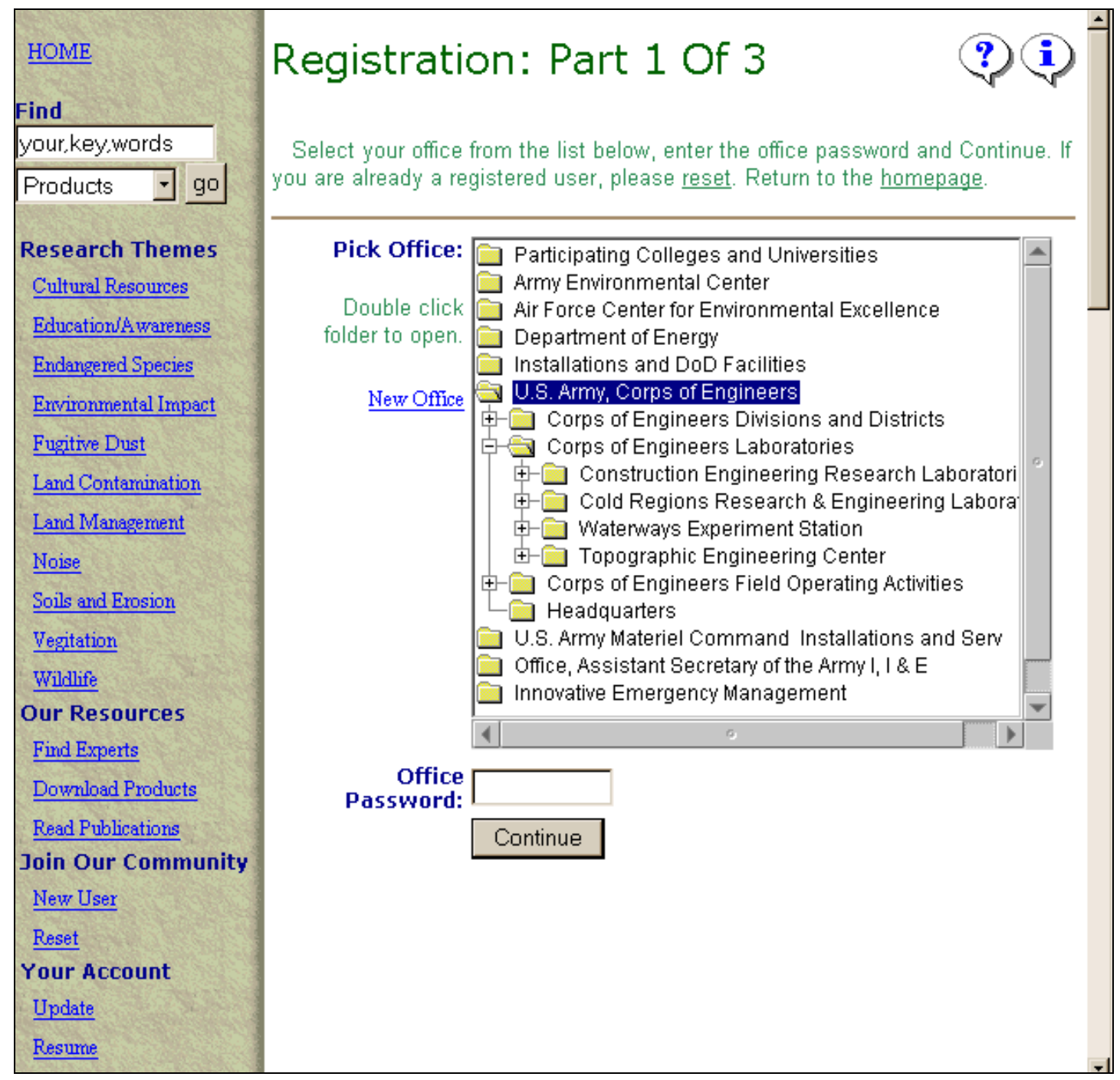

Figure 9. Select an office.

As the user enters the personal data, he/she may want to select a job series that is not on the list. If so, the user may add that new series.

After entering the personal data, the system checks to make sure that no duplicate user names exist. If duplicates exist, the user is asked to change his/her information or contact the system administrator. If no duplicates exist, then the system creates the user's personal password, sets the user's security key, and sends a confirming e-mail to the address provided by the user. 
Individuals registering in offices designated as PAO, program management, or research offices are provided with a default set of permissions related to those offices.

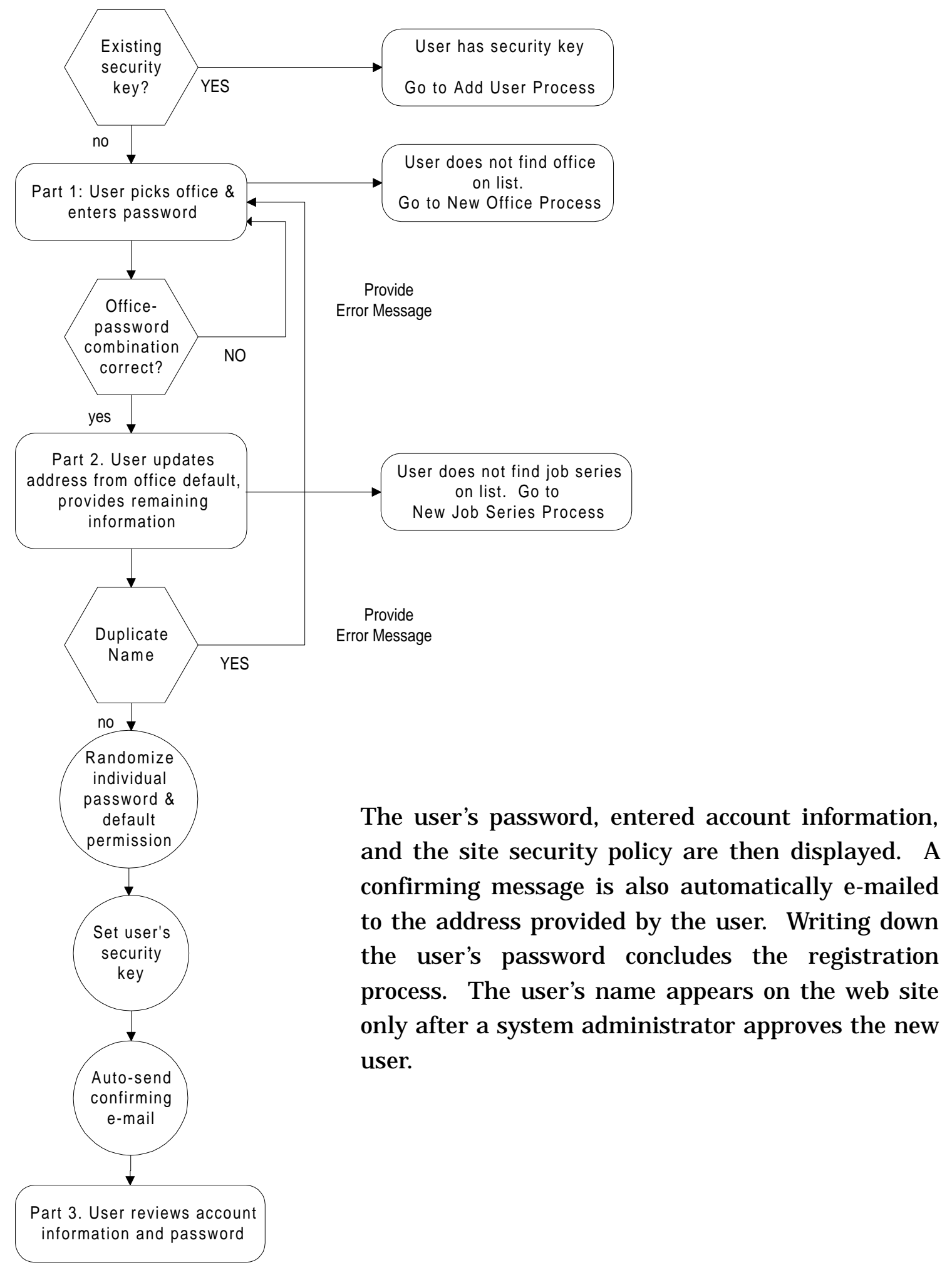

Figure 10. Public user process. 


\section{Developer Process: New Office}

If users attempt to register but are unable to find their office, they may add a new office. Figure 11 shows the first of two pages required to add a new office to the list.

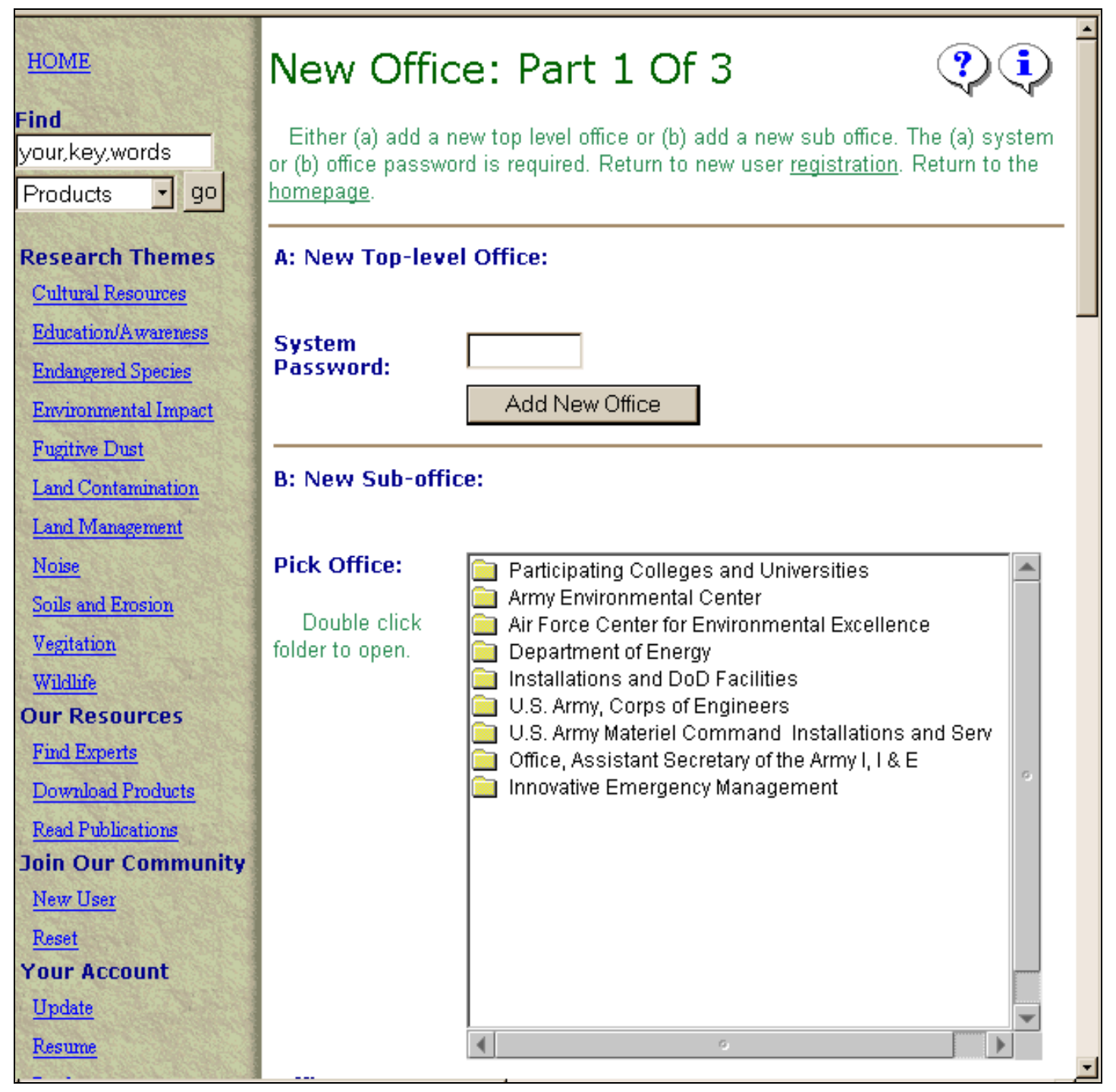

Figure 11. Add new office.

Figure 12 shows the process to add a new office. In Part 1, the user picks the parent organization or identifies the office as a new top-level office. The system administrator holds the password for the creation of new top-level offices. A suboffice password is sufficient to create sub-level offices. 
Once the password is correct, and the system has confirmed that the office has not been duplicated elsewhere, then a new password is generated for that office and the new office is created.

The system sends an e-mail message to all system administrators informing them of the new addition to the office database.

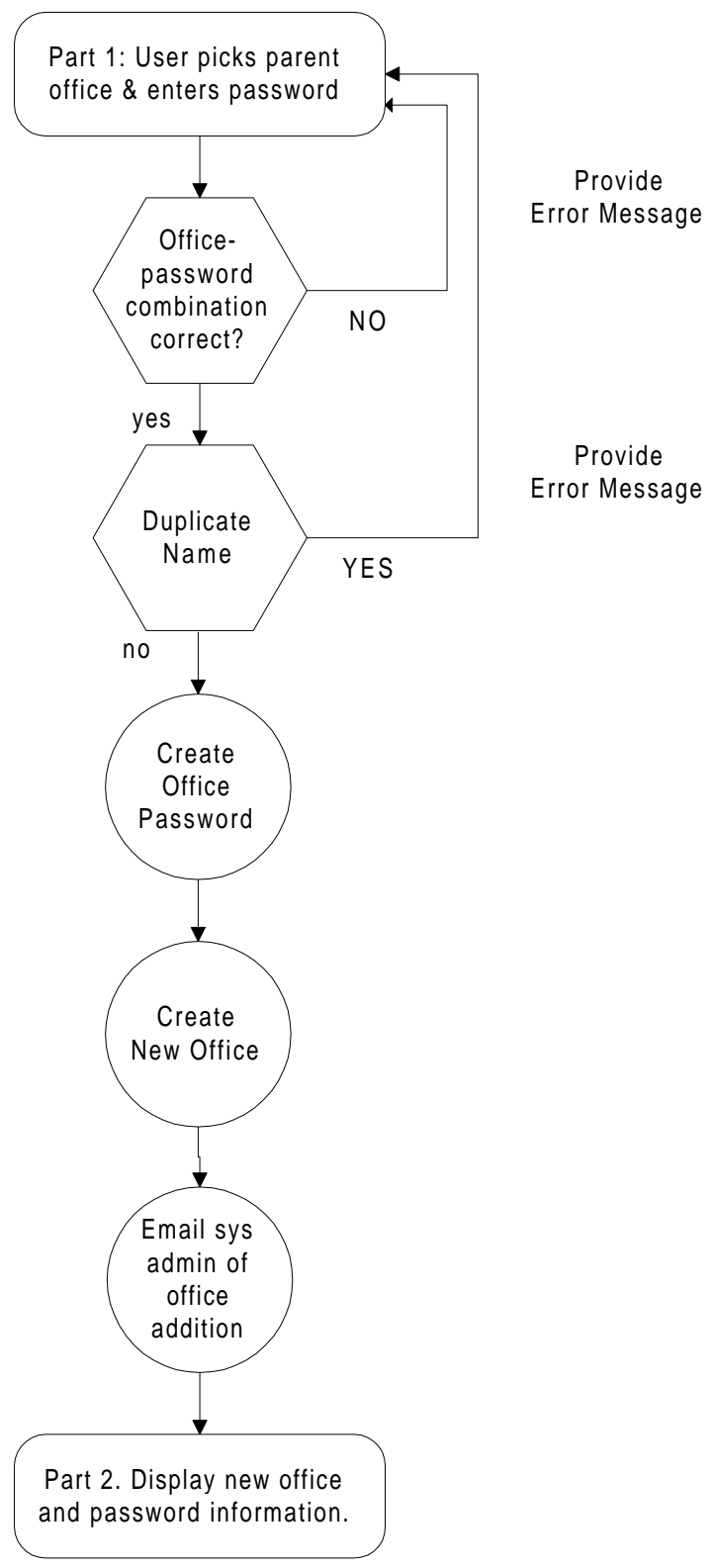

Figure 12. Process to add new office. 


\section{Developer Process: New Job Series}

If users attempt to register but are unable to find their correct $F$ ederal job series designation, they may add the new series. Figure 13 shows the page needed to add a new job series.

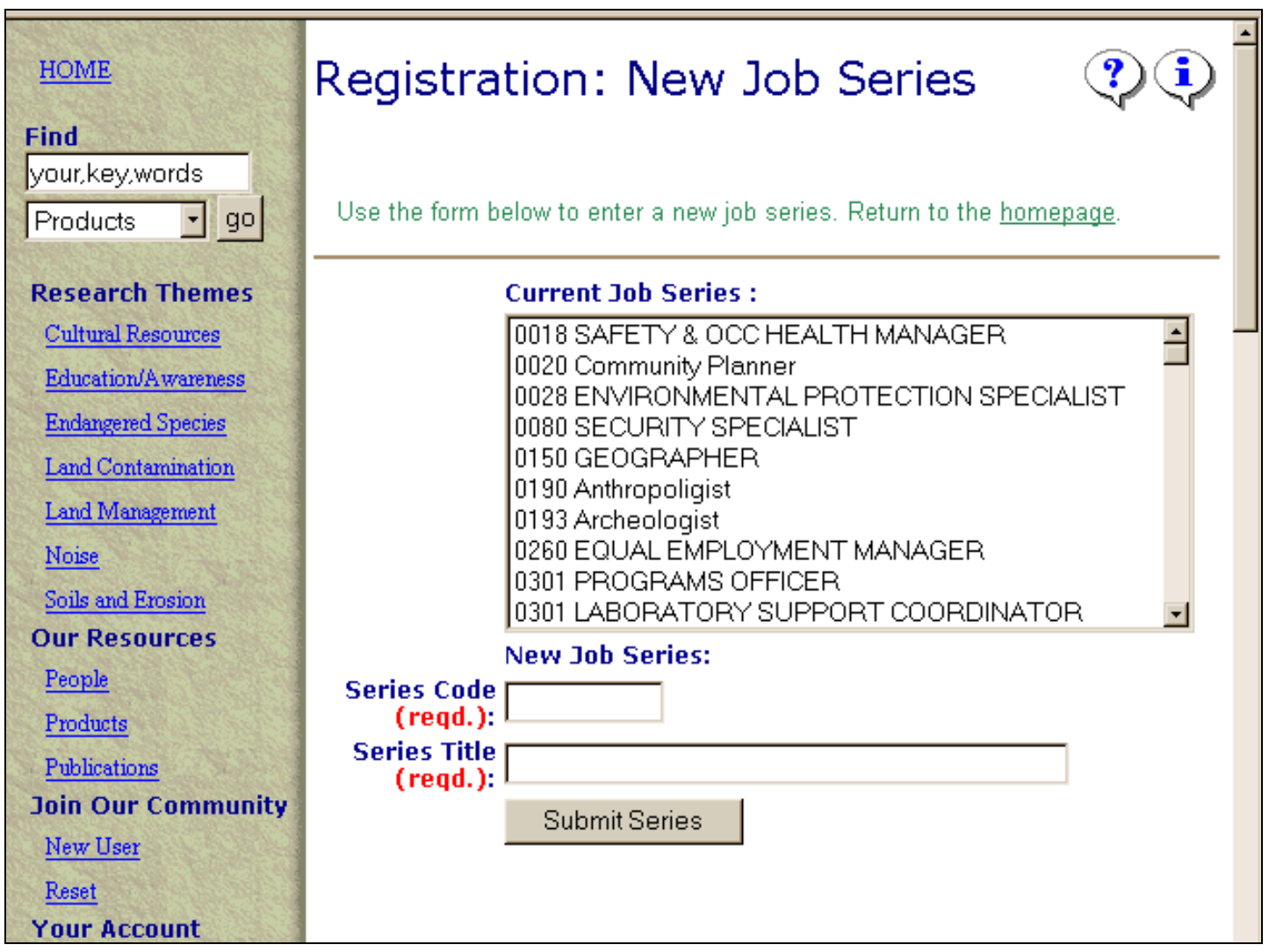

Figure 13. New job series.

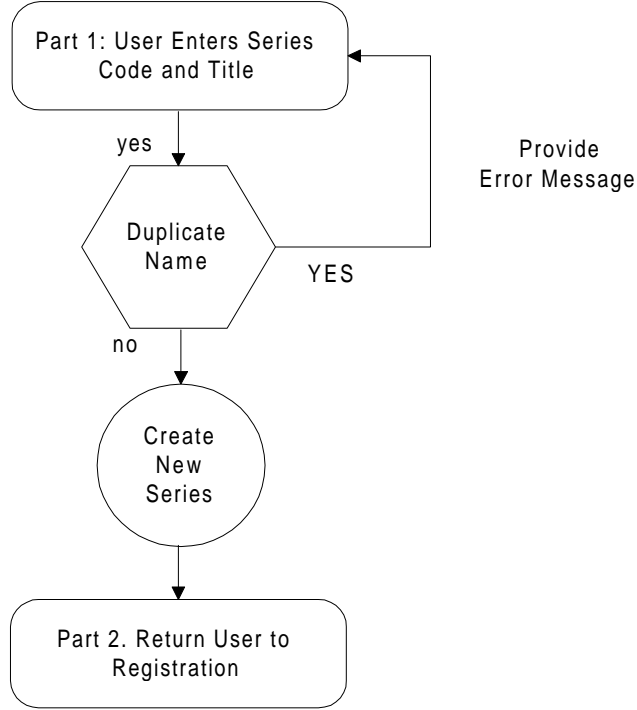

The procedure for adding a new job series, shown in Figure 14, is very simple. The user can add any new series, provided that the entries are not duplicates of information previously provided.

Figure 14. Process to add new job series. 


\section{Developer Process: Add New User}

Existing users may also add new users. This process (shown in Figure 15) is essentially the same as new user registration. There are, however, two important differences between the "add a new user" and the "new user" process.

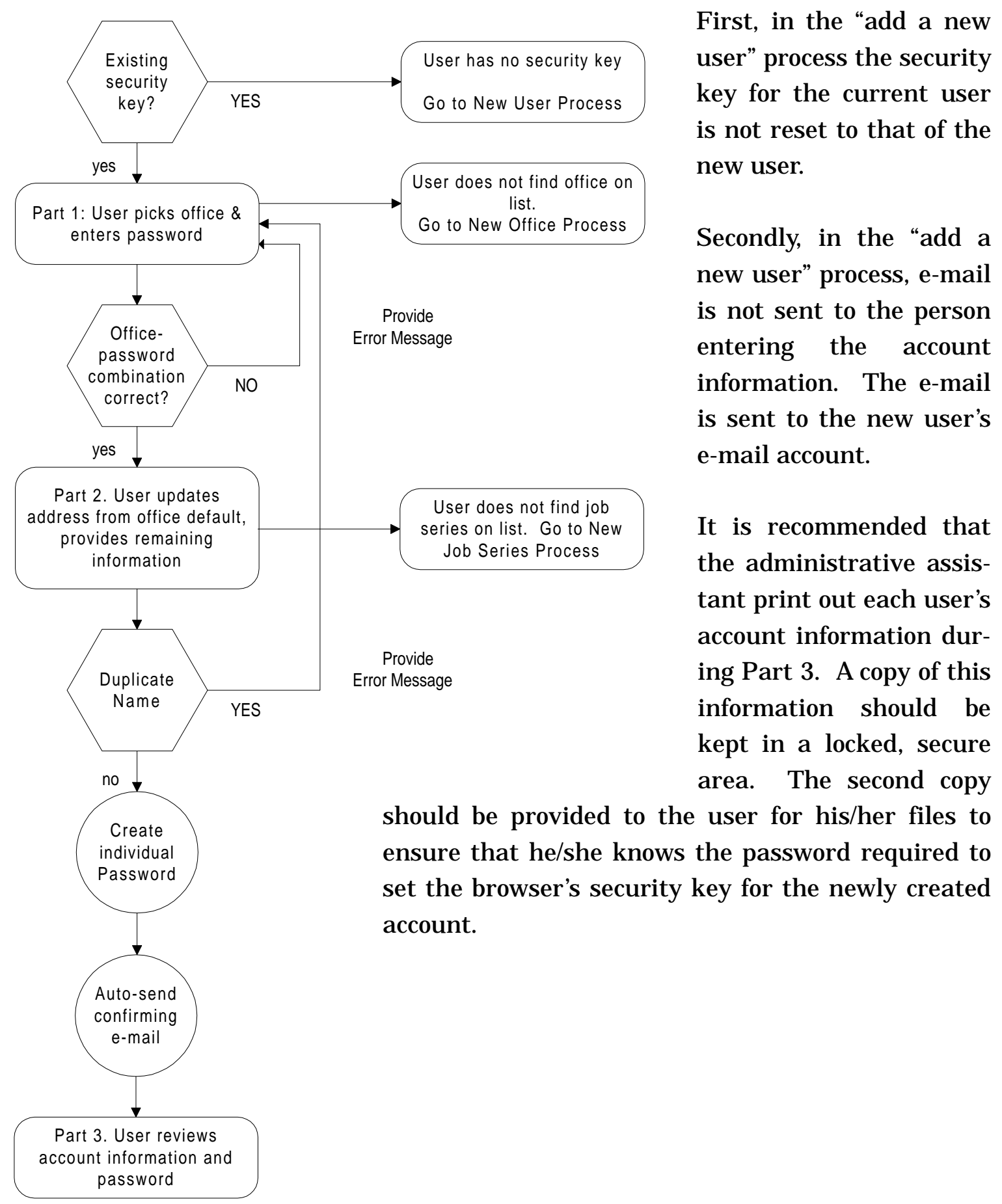

Figure 15. Process to add new user. 


\section{Developer Process: Reset User Security Key}

When users change machines, upgrade their browsers, or perform certain browser caching functions, the site security key may need to be reset. If a user was previously registered but suddenly the site no longer knows the user, then the user has somehow removed his/her security key.

The two-part process, shown in Figure 16, is used to reset the user's security key. In Part 1, the user selects his/her name from the list of all site registrants and provides the proper password. If the password is correct, then the system resets the user's security key and sends an automated e-mail confirming this action to the user's account e-mail address.

In Part 2, the user reviews his/her account information and may print out a copy for future reference.

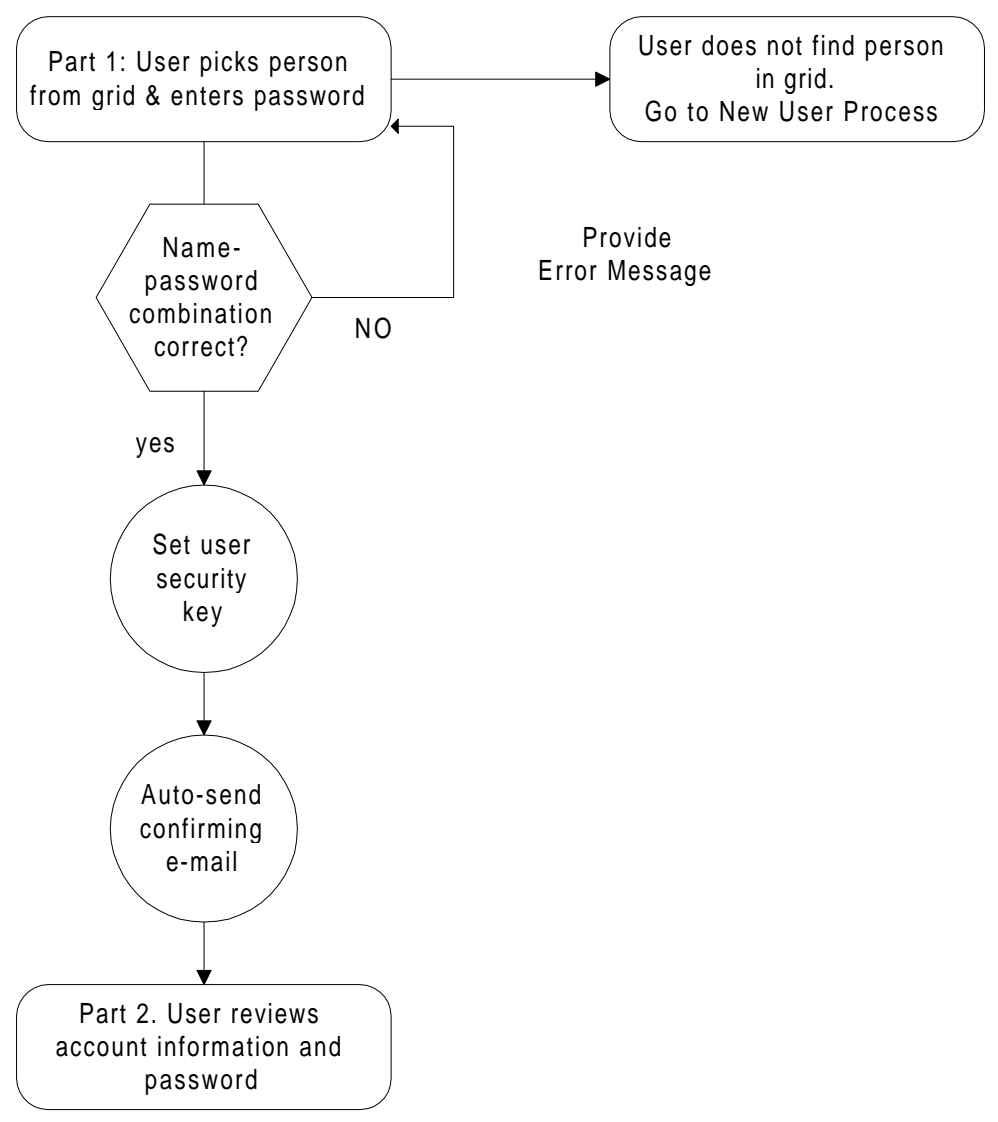

Figure 16. Process to reset user security key. 


\section{Developer Process: Update User Account}

Registered users will see an option to change their account information. Figure 17 shows the account update page. Account updates occur in two parts. In Part 1 , the user changes the data shown in the figure and submits the form. If the password is correct, then the user's information is updated. Note that users must type in their passwords to verify that they are who they claim to be, regardless of the security settings of the browser. Figure 18 shows the process to update a user's account.

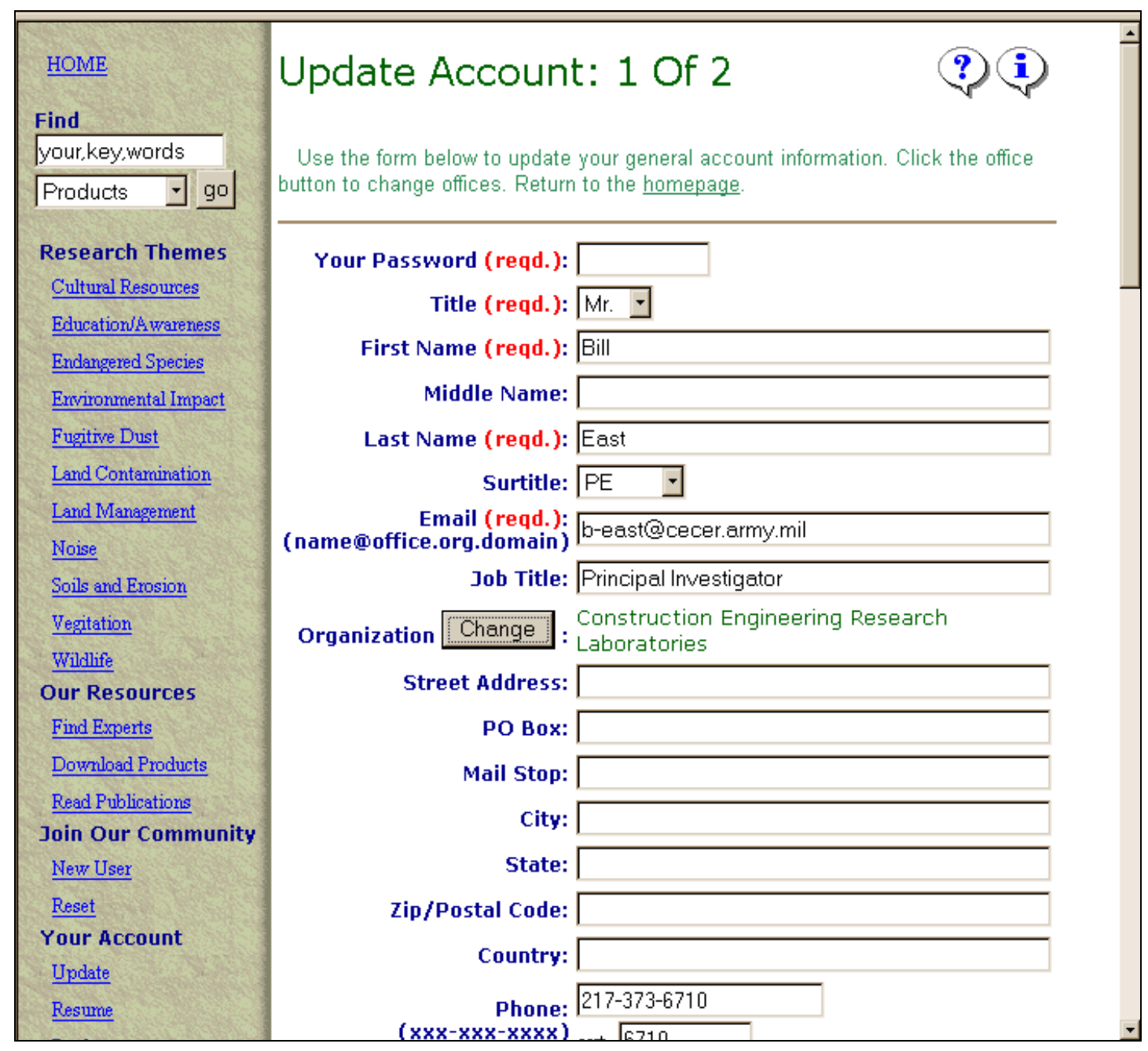

Figure 17. Update user account.

The system creates a new personal password and sends an automatic e-mail to the e-mail address provided. 


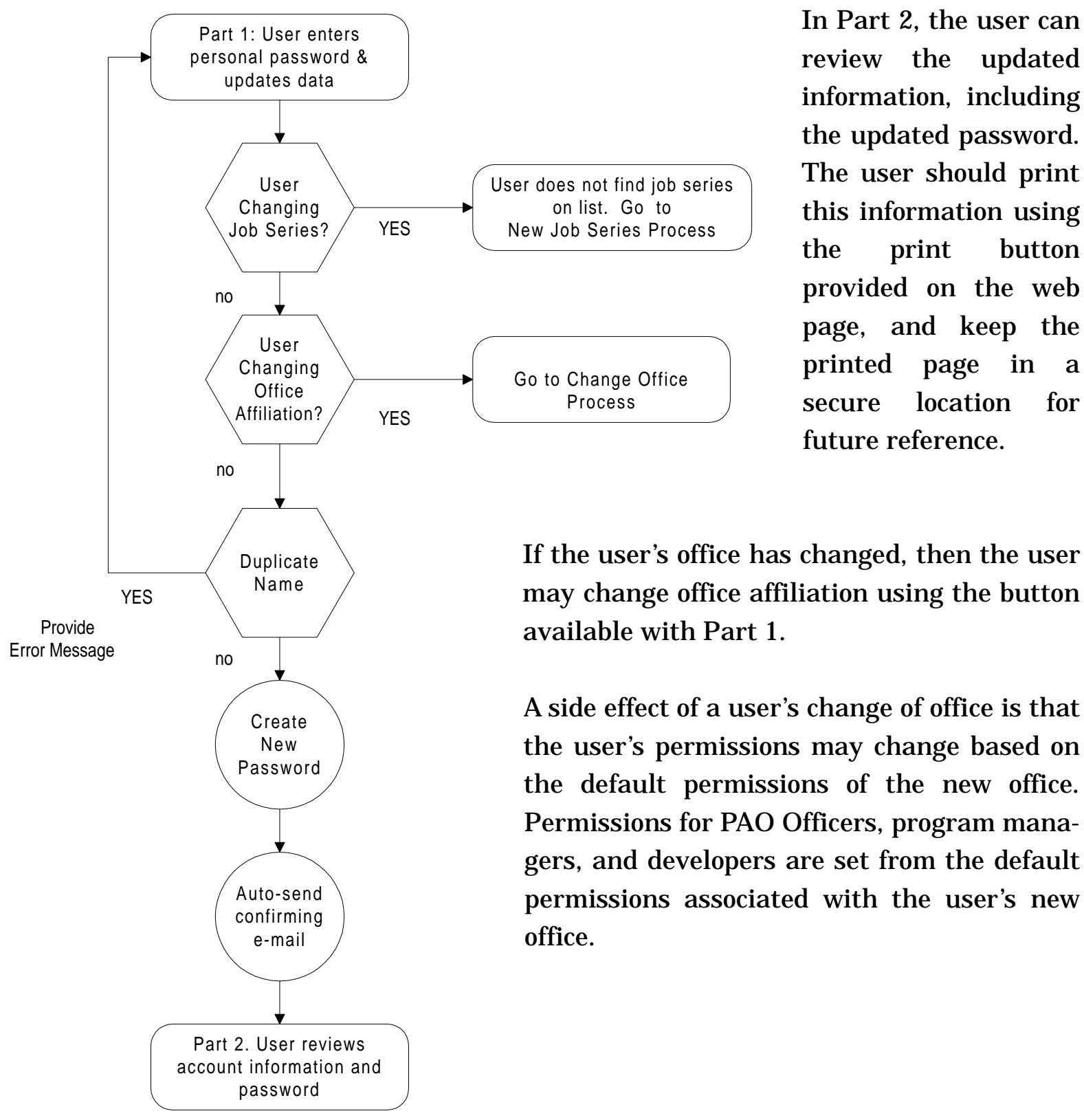

Figure 18. Process to update user account. 


\section{Developer Process: User Resume}

Once a user account has been created and approved, the user may begin to enter his/her on-line resume. Figure 19 shows the "Update Resume" page with a completed resume. The five resume sections are Objective, Education, J ob History, Professional Registrations/Professional Affiliations, and Awards and Honors. Each of these sections may be selected directly from the U pdate Resume page. Once selected, individual data add and update pages are provided to allow users to update specific information.

\begin{tabular}{|c|c|}
\hline$\underline{\text { HOME }}$ & \multirow{4}{*}{$\begin{array}{l}\text { Update Resume } \\
\text { Update your resume using the links below. Change your name, office, phone, } \\
\text { etc.. with update account. Your resume was last updated on 31-Mar-99. Your } \\
\text { resume approved by Sara Ort on 23-Mar-99 View your on-line products or } \\
\text { publications. Return to the homepage. }\end{array}$} \\
\hline Find & \\
\hline your,key,words & \\
\hline Products $\exists$ go & \\
\hline Research Themes & \multirow{8}{*}{$\begin{array}{l}\text { Mr. Bill East, PE } \\
\text { Principal Investigator, Engineering Processes Division (CECER- } \\
\text { PL-E), Job Series: } 1550 \text { Computer Scientist, Work Phone: } \\
217-373-6710 \text { (ext: } 6710 \text { ), FAX: } 217-373-6724 \text {, EMail: b- } \\
\text { east@ocecer.army.mil } \\
\text { Objective: } \\
\text { (edit) To create and deliver leap-ahead technologies to the Corps of } \\
\text { Engineers and the U.S. Construction Industry that improve the } \\
\text { cost, time, quality performance of the built environment. }\end{array}$} \\
\hline Cultural Resources & \\
\hline Education/A wareness & \\
\hline Endangered Species & \\
\hline Land Contamination & \\
\hline Land Management & \\
\hline Noise & \\
\hline Soils and Erosion & \\
\hline Our Resources & \multirow{4}{*}{$\begin{array}{l}\text { (add) } \\
\text { (edit) } \text { Ph.D. Civil Engineering, University of Illinois, expected } \\
\text { M.S. Civil Engineering, University of Illinois } \\
\text { B.S. Civil Engineering, VA Tech }\end{array}$} \\
\hline People & \\
\hline$\underline{\text { Products }}$ & \\
\hline Publications & \\
\hline Join Our Community & \multirow{3}{*}{ (add) Registration: } \\
\hline$\underline{\text { New User }}$ & \\
\hline$\underline{\text { Reset }}$ & \\
\hline Your Account & \\
\hline Update & (add) Job History: \\
\hline$\frac{\text { Resume }}{\text { Products }}$ & $\begin{array}{l}\text { (edit) Lecturer, University of Illinois, Department of Civil } \\
\text { Engineering }\end{array}$ \\
\hline Publications & Taught one-third of the Introduction to Construction \\
\hline Supervise Resumes & $\begin{array}{l}\text { Management course at the University of Illinois until } 1995 \text {. } \\
\text { The course used a computer-based tutorial program to teach }\end{array}$ \\
\hline & Arrow Diagramming developed through Uofi grant and \\
\hline
\end{tabular}

Figure 19. Update resume. 
The procedure to add to resumes and update resume sections is shown in Figure 20. From the main view resume page, those sections of the resume that have been entered are shown. The user may select any of the sections to add, edit, or delete data.

In addition to changing specific types of information about a resume, the "Developer" may also change the databases containing the list of: valid degree types, valid professional registration types, and valid award types. Maintaining such information in the system database ensures that a consistent set of information is captured for all technical domains across all ERDC sites.

Specific figures showing the pages for changing the data contained in the resume are not included in this report. They are quite simple and have no additional coding or business processes associated with them.

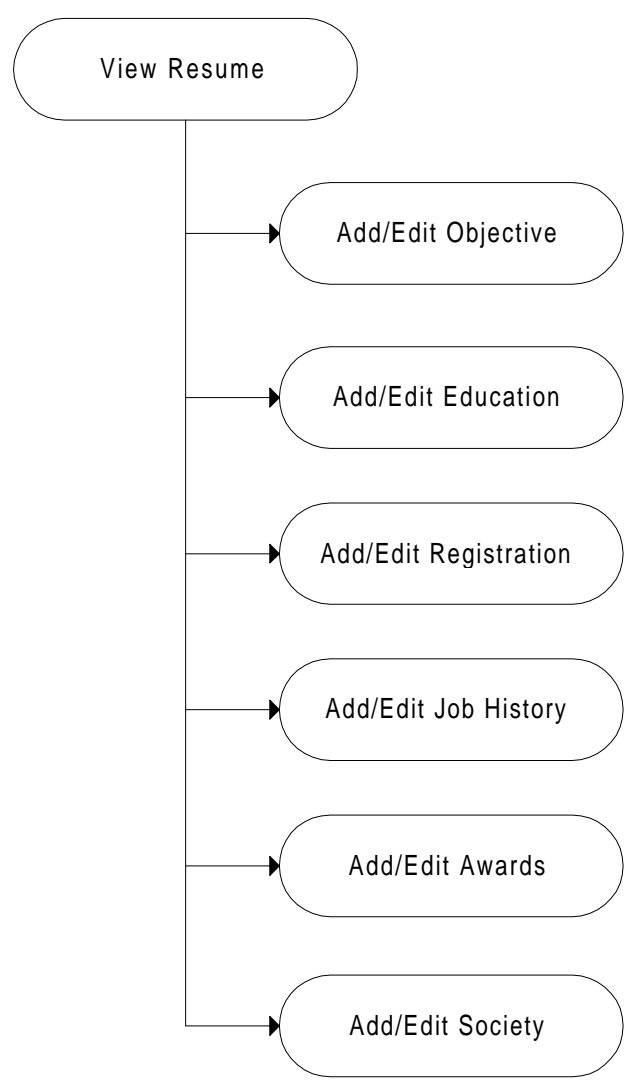

Figure 20. Process to update a resume. 


\section{Developer Process: Add Publications}

The four parts to adding a publication are: Selecting the Publication Type, Selecting the Publisher, Selecting the Publication POCs, and entering the publication citation, abstract, or any related on-line documents. Figure 21 shows the first part of the process.

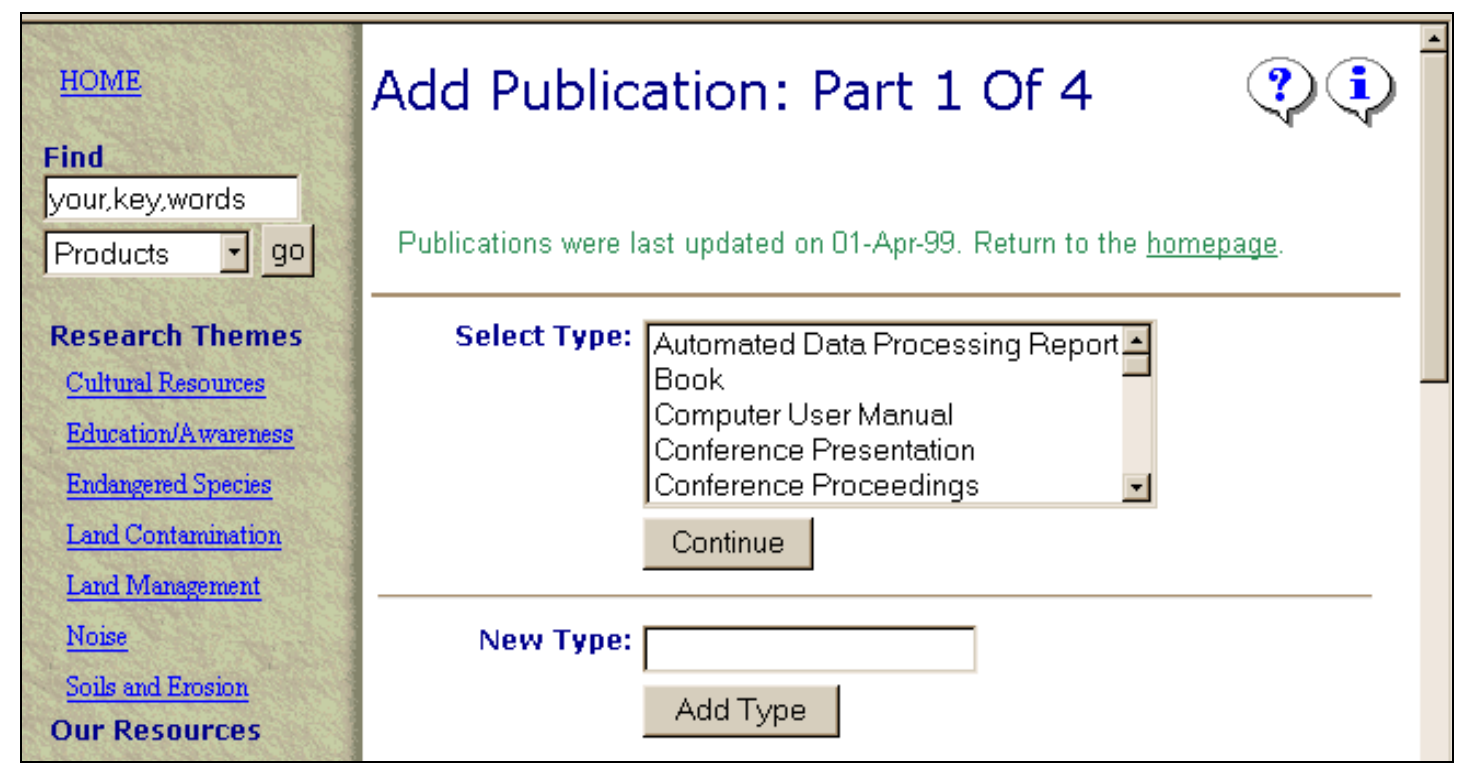

Figure 21. Select publication type.

As information on a new publication is added, the data is stored in a temporary table. Using this temporary table allows the user to backtrack through the first three steps of this process without losing any information.

Figure 22 shows the second part of the process, selecting a new publisher. If the publisher is not on the current list, the developer may add the name and location of the publisher.

Figure 23 shows the third part of the publication process, selecting POCs. POCs are sel ected to allow users of the system to find out whom to contact in regard to a particular publication or product. Since contractors or persons no longer with an organization may write some publications, the POC list allows all those people associated with a publication to be linked regardless of their inclusion in the author list. 


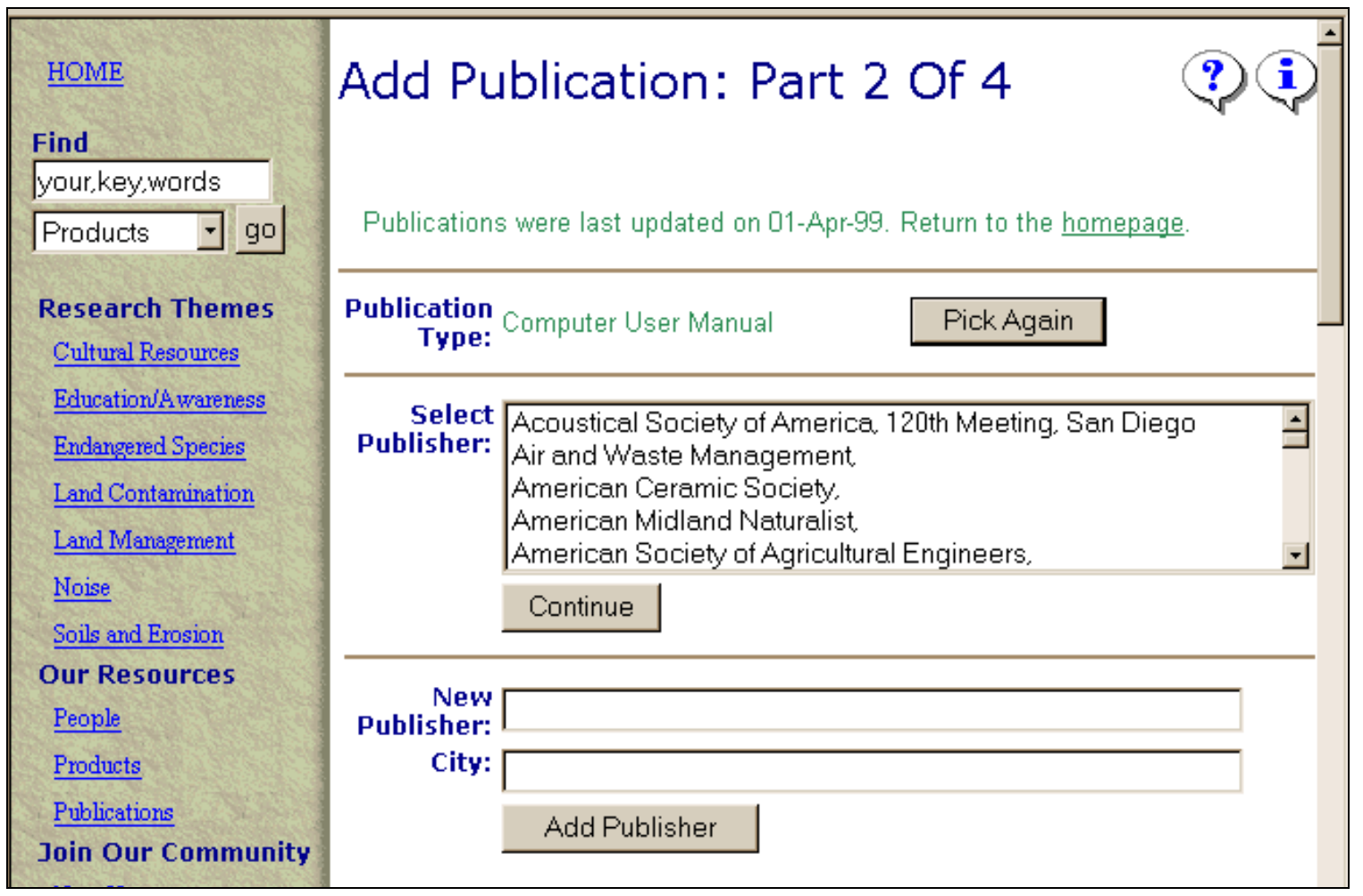

Figure 22. Select publication publisher.

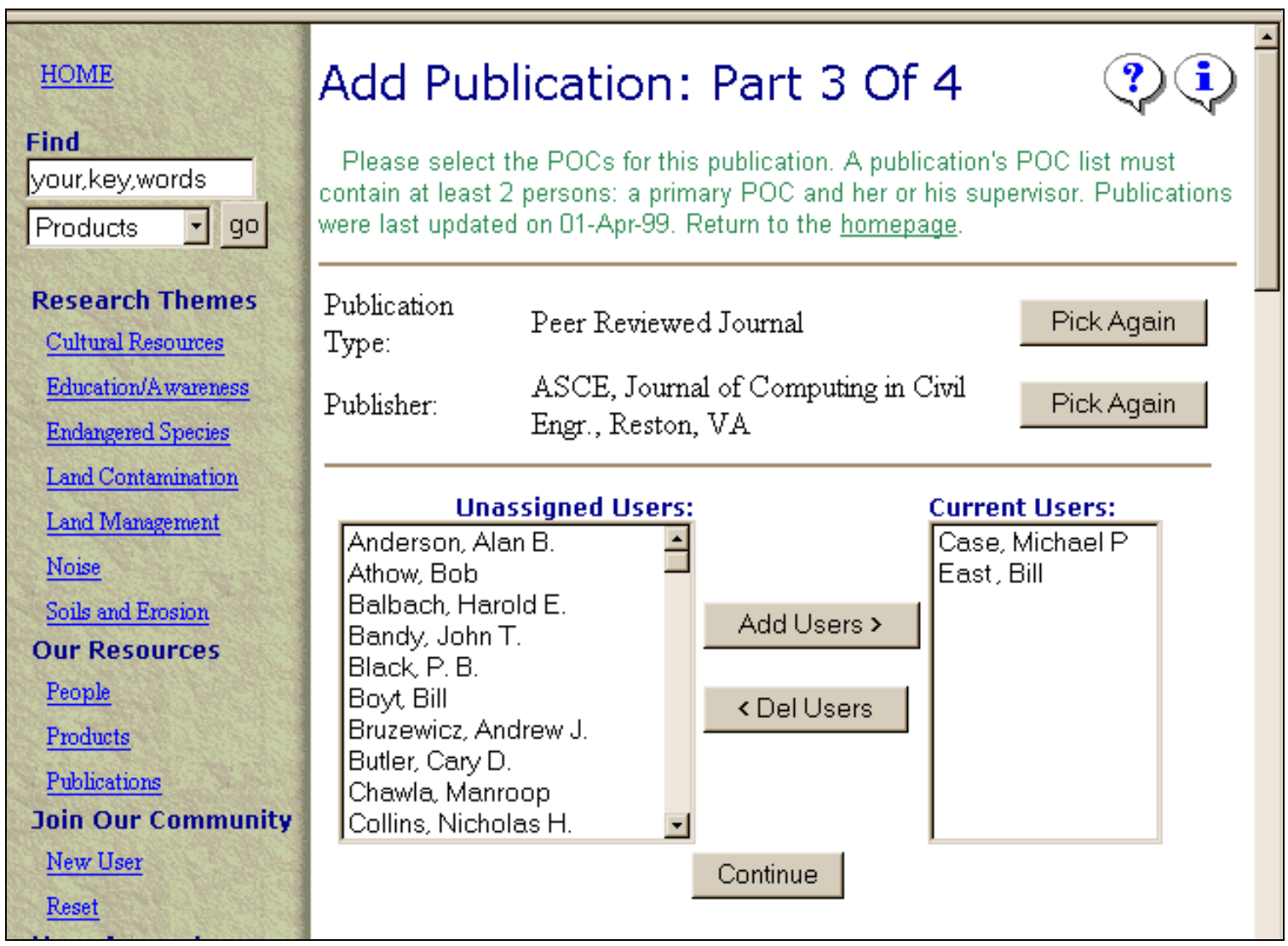

Figure 23. Select publication POCs. 
The order of the POCs in the list will also translate into the order of the default set of authors for the publication. Figure 24 shows a portion of the page needed to add a new publication. The results of parts 1,2 , and 3 are shown by the Publication Type, Publisher, and POC data, respectively. The developer may change the selections using the appropriate "Pick Again" button.

It is important to note that, at this point, the "Pick Again" button will disregard any information currently entered in the publication citation and require the user to return through subsequent parts of the publication addition process to return to this page. This means that users who enter a long abstract and do not save the publication prior to returning to change the POCs will lose the information entered.

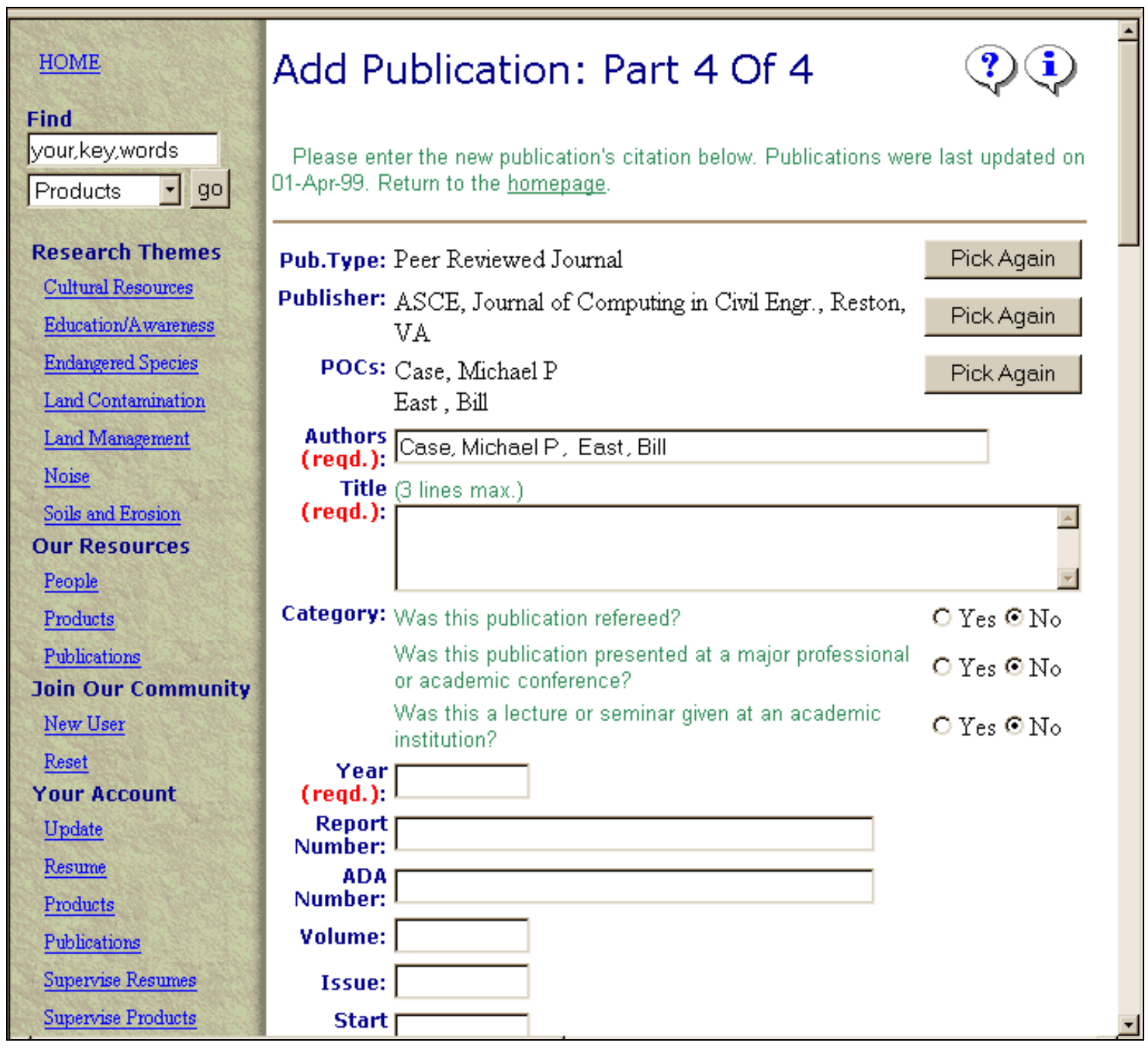

Figure 24. Add publication citation. 


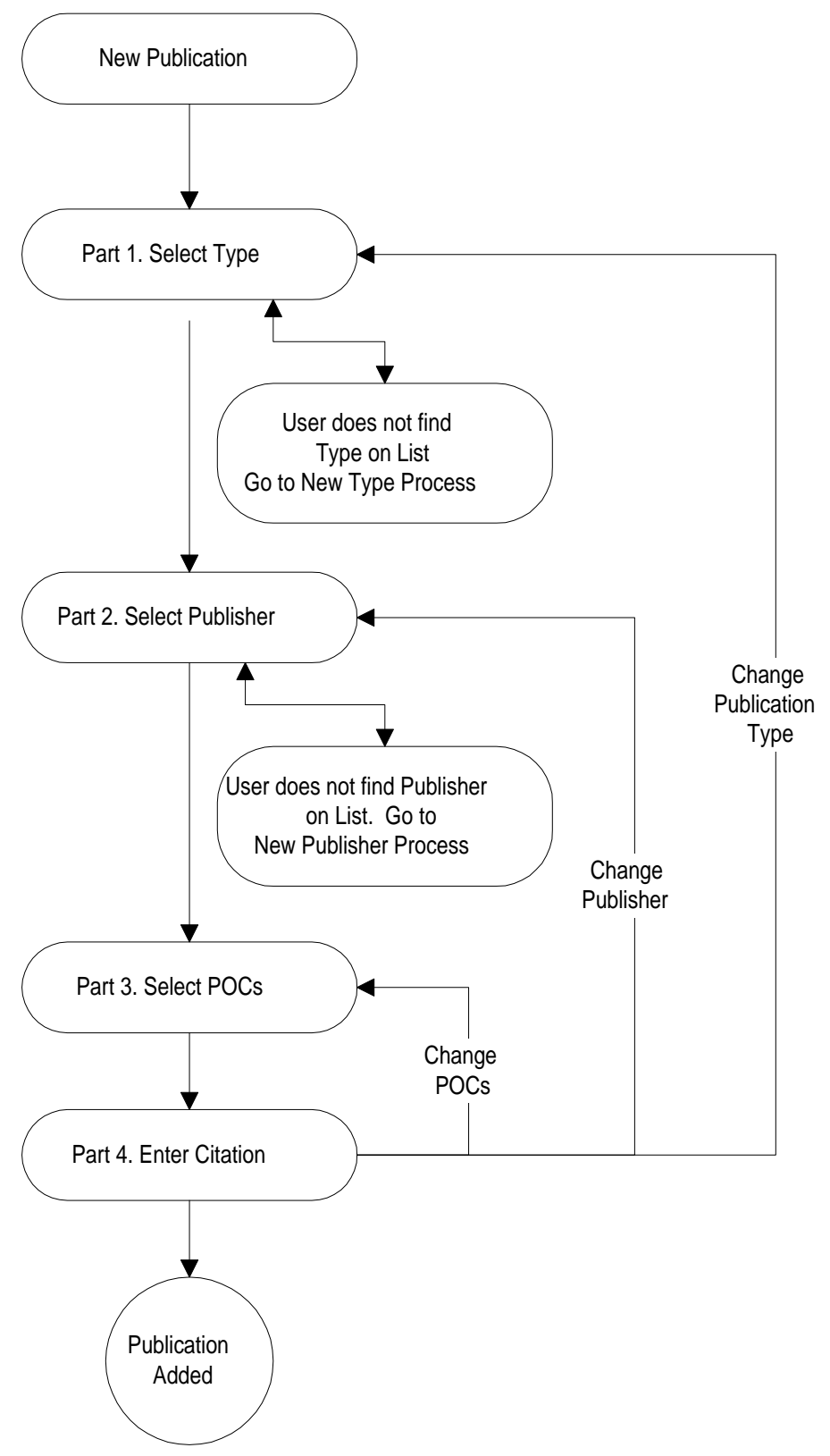

The three "yes/no" questions shown to the right of the "Category" heading are provided to allow easy extraction of publication data for management level reporting. Reports developed using these categories support various data calls required during the year.

Figure 25. Process to add new publication. 


\section{Developer Process: Edit Publications}

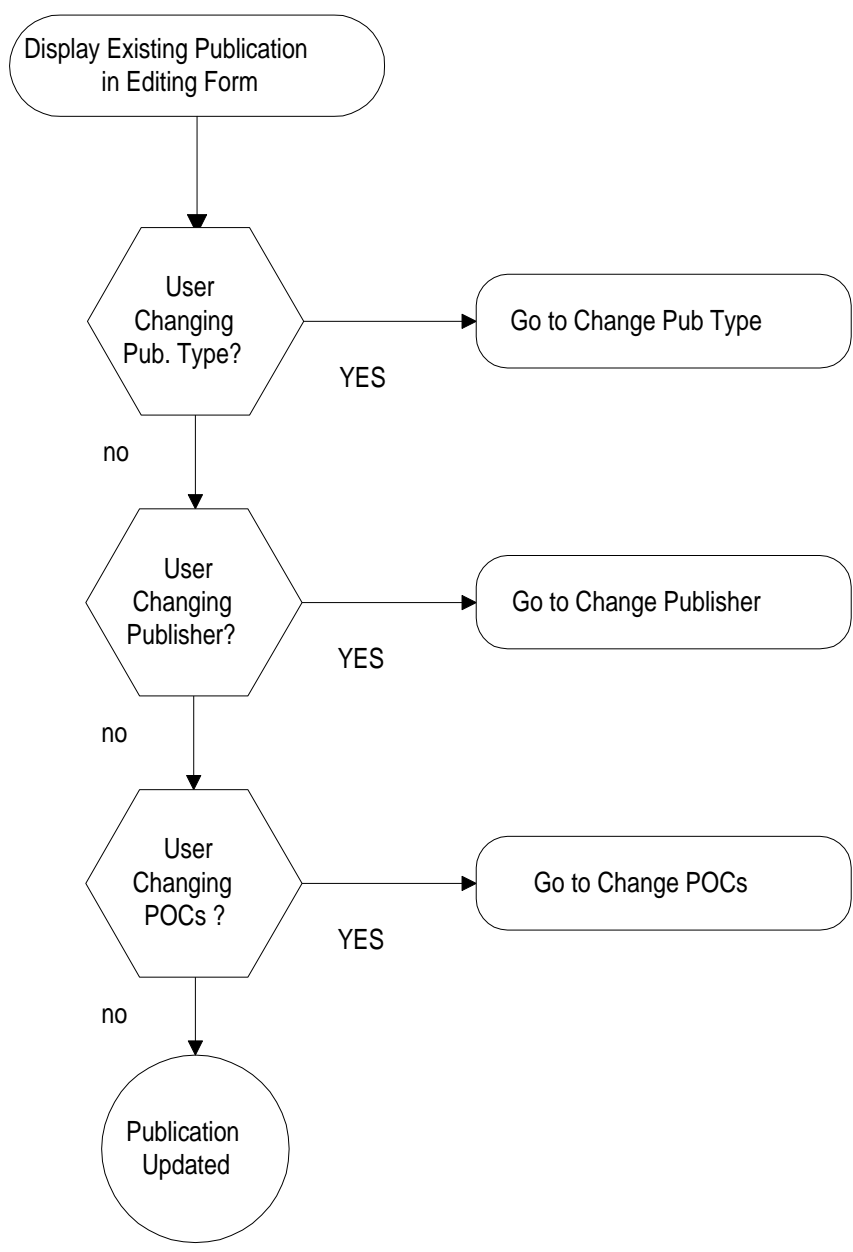

Any "Developer" assigned to the publication as a POC may edit the publication once it has been created. The procedure to edit a publication is shown in Figure 26.

Figure 26. Procedure to edit a publication. 


\section{Developer Process: Add Products/Services}

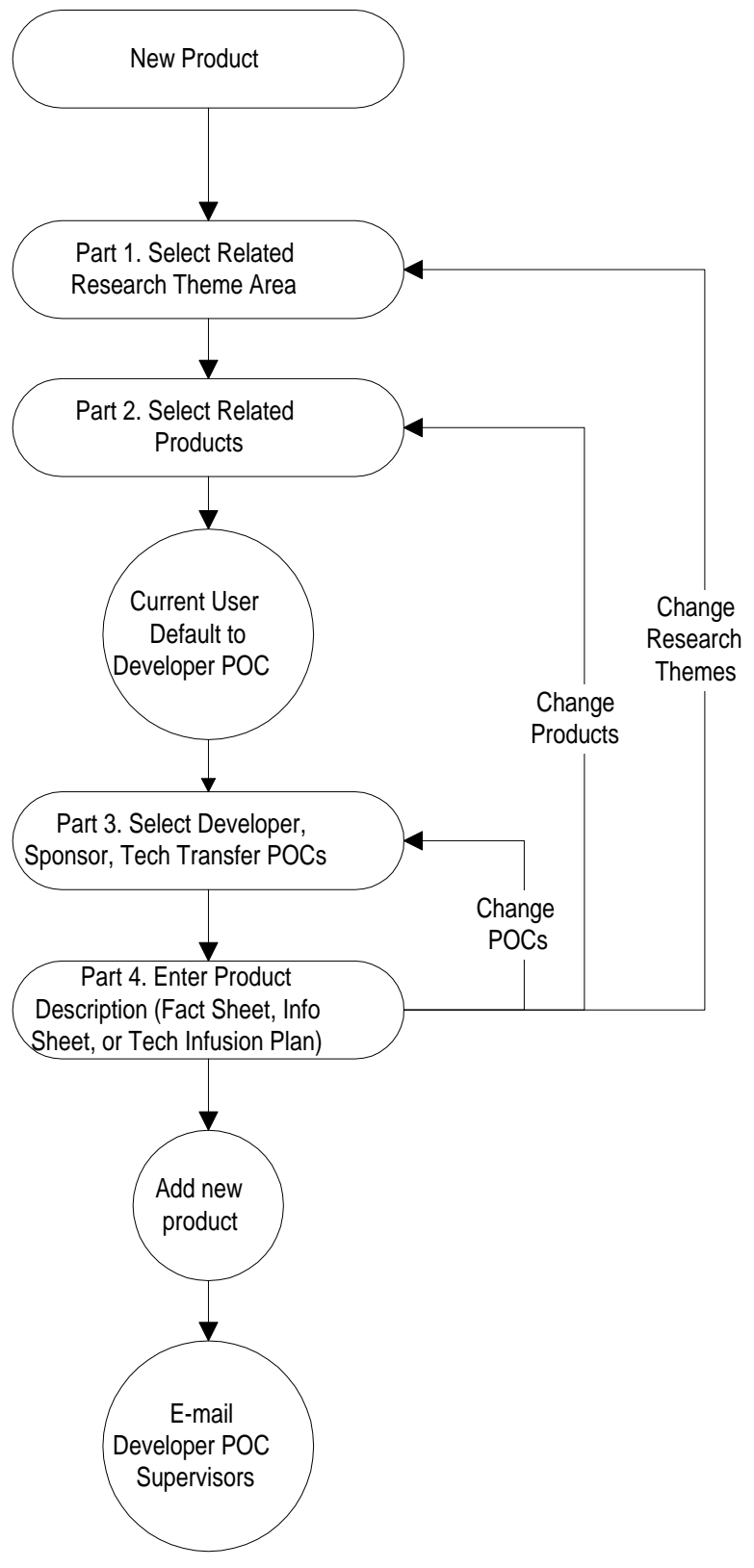

Figure 27. Procedure to add products/services.
Products/Services in the TIPS site refer to a broad range of materials that could be provided to users. These materials include: (a) descriptions of (and links to obtain) software or hardware, (b) descriptions of capabilities or services provided by researchers, or (c) informational products that provide historical and related background information. Figure 27 shows the four "Developer" steps needed to add products.

Initially, the "Developer POCs" identified are the only ones who may review product descriptions. E-mail is automatically sent to supervisors to alert them that a new product must be approved. Once approved by the supervisory personnel for any of the "Developer POCs," the product is available for public consumption. 


\section{Supervisor Process: Approve Resumes}

Review and subsequent approval of draft resumes are required before a "Developer" resume is made available for public viewing. TIPS has a menu selection, only available to supervisors, called "Supervise Resumes." Figure 28 shows one example page. On this page all members of an organizational level to which a "Supervisor" belongs are displayed.

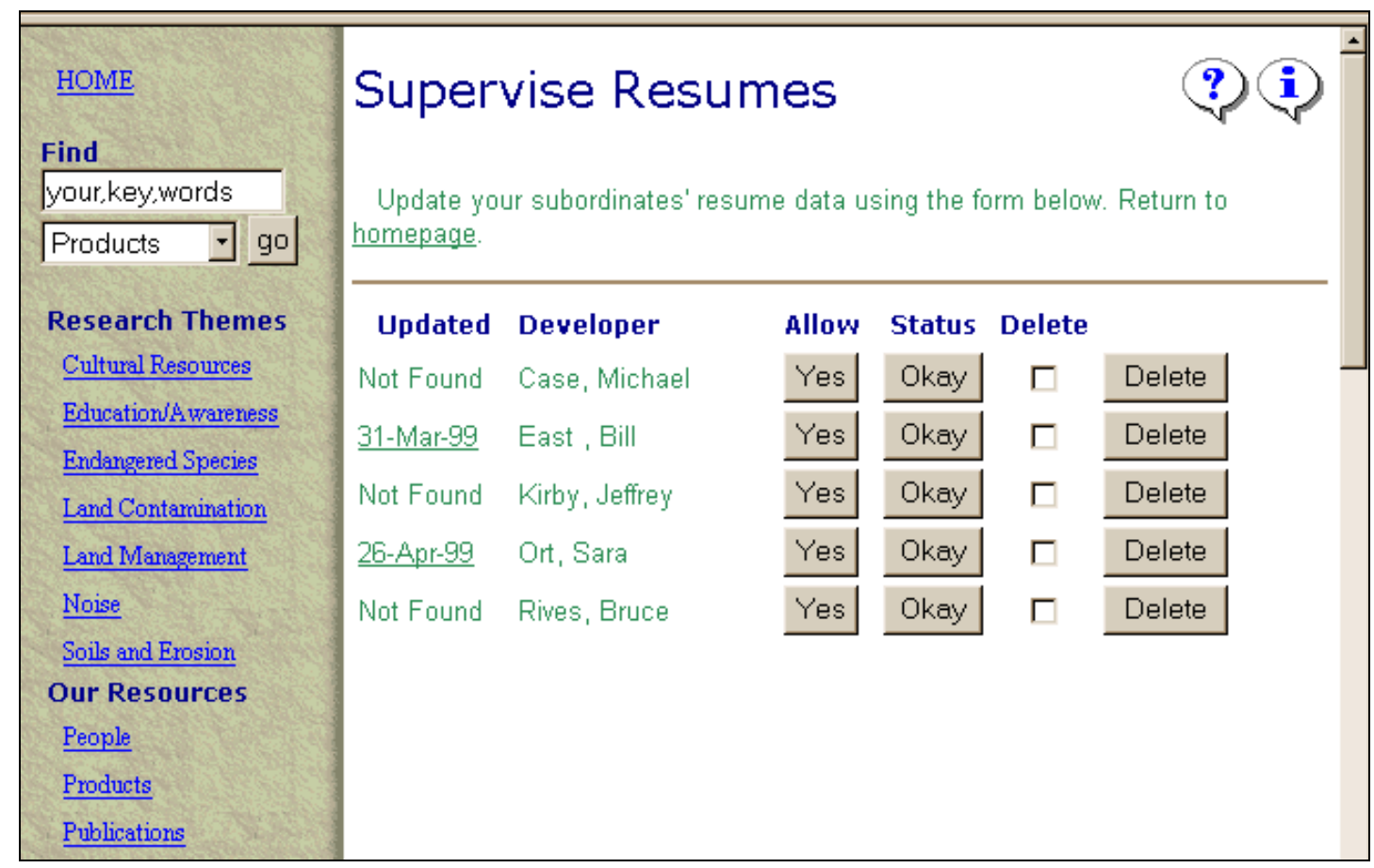

Figure 28. Approve resumes.

The table shown in Figure 28 provides all needed information and links required for supervisory approval of resumes. The first column indicates when the last resume update for each person was completed. If a resume exists, then the supervisor may review the resume by following the link provided.

To the right of the "Developer" column is a column that allows users to create resumes. When the button in the "Allow" column shows "Yes," the developer can see the "Resume" link under the "Your Account" section of the left-hand menu. If the allow button is toggled to the "No" position, then the user cannot change his/her resume, because there is no link to the required page.

A side effect of a supervisor changing the user's status to "allow" is that the user becomes an "approved" user. Since resumes also include publications, user permissions to add publications are also enabled. The user's ability to view on-line reports is also turned on as a side effect of approving a user on this page. 
If the supervisor turns the user's allow status to "no," then the user can no longer update his/her resume and the user's resume is also disapproved.

While a user may add a resume, that resume may need some revision. There fore, another toggle button is provided under the "Status" column. If the status is "Okay" then the public may view the user's resume. When the supervisor "okays" a resume, both the "allow" and "approve" resume switches are thrown.

If the status is changed to "Deferred," then the user's resume is no longer visible to the public. The resume is still accessible to the user and the supervisor for subsequent modification and approval.

If a supervisor determines it is needed, then the "Delete" button may remove all resume information for the user. To delete all resume information, the supervisor must click the check box next to the resume delete button and then click the delete key.

\section{Supervisor Process: Approve Products}

Prior to being viewed by the public, all product descriptions must be approved by a supervisor. Figure 29 shows the product approval page. All of the developers in an office are listed, in alphabetical order according to last name, with all of their current product listings.

Across from each person is the option for the "supervisor" to determine if the person is to be allowed to add new products or not. If the "Prod. Allowed?" toggle is set to "Yes" then the user will see the "Products" link under their "Your Account" menu selection.

A side effect of a supervisor changing the user's status to "allow" is that the user becomes an "approved" user. User permissions to add publications are also turned on as is the user's ability to view on-line reports.

If the toggle is set to "No," then the user will not see the products link and will not be able to add product information.

Evaluation of each of the products must be accomplished separately from the approval of individuals to add product information, since persons may be listed as a product "Developer POC," and thus have products listed under their names, but not be able to add new products themselves. 
Individual products may be evaluated by any supervisor of any "Developer POC." Products may be "Approved" for public distribution or "Deferred." Supervisors may also delete individual products, provided that the confirming check-box is shown. Products that have cross-organizational POCs will have a supervisor in each of these organizations with the option to "Defer" a product listing.

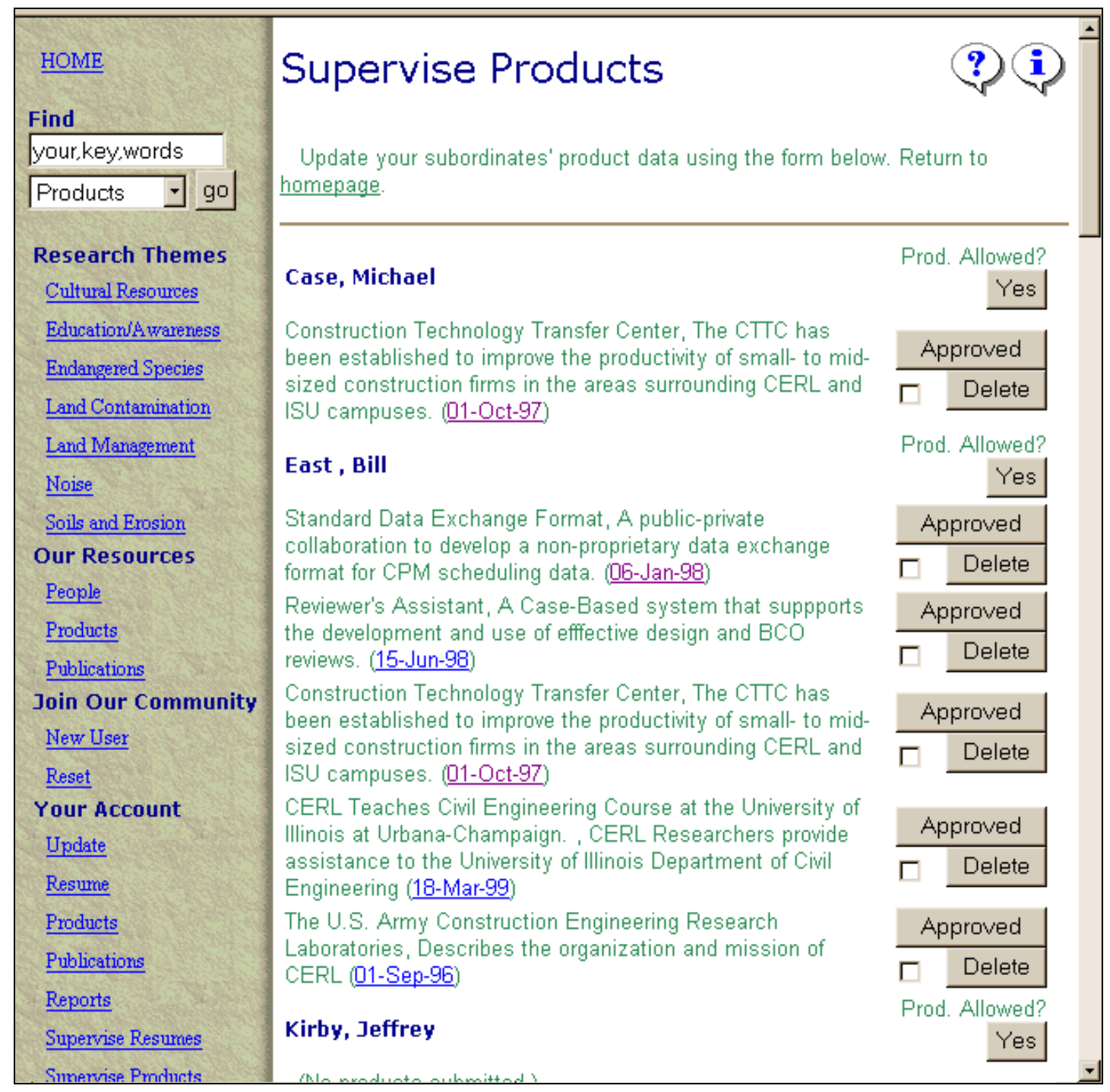

Figure 29. Approve products. 


\section{Supervisor Process: Approve Publications}

Figure 30 shows the page provided to supervisors to allow them to authorize publications for individuals in their organization and approve or delete individual publications. The toggle button under "Pubs Allowed?" changes the visibility of the user's link to add or edit publications. Those users who do not have publications "allowed" do not see the link to publications under "Your Account" and cannot add new publications. Users for whom publications are "allowed," see the publications link and can, therefore, add or update publications.

A side effect of allowing user publications is to approve the user for public access. In addition, the user's ability to view on-line reports is turned on.

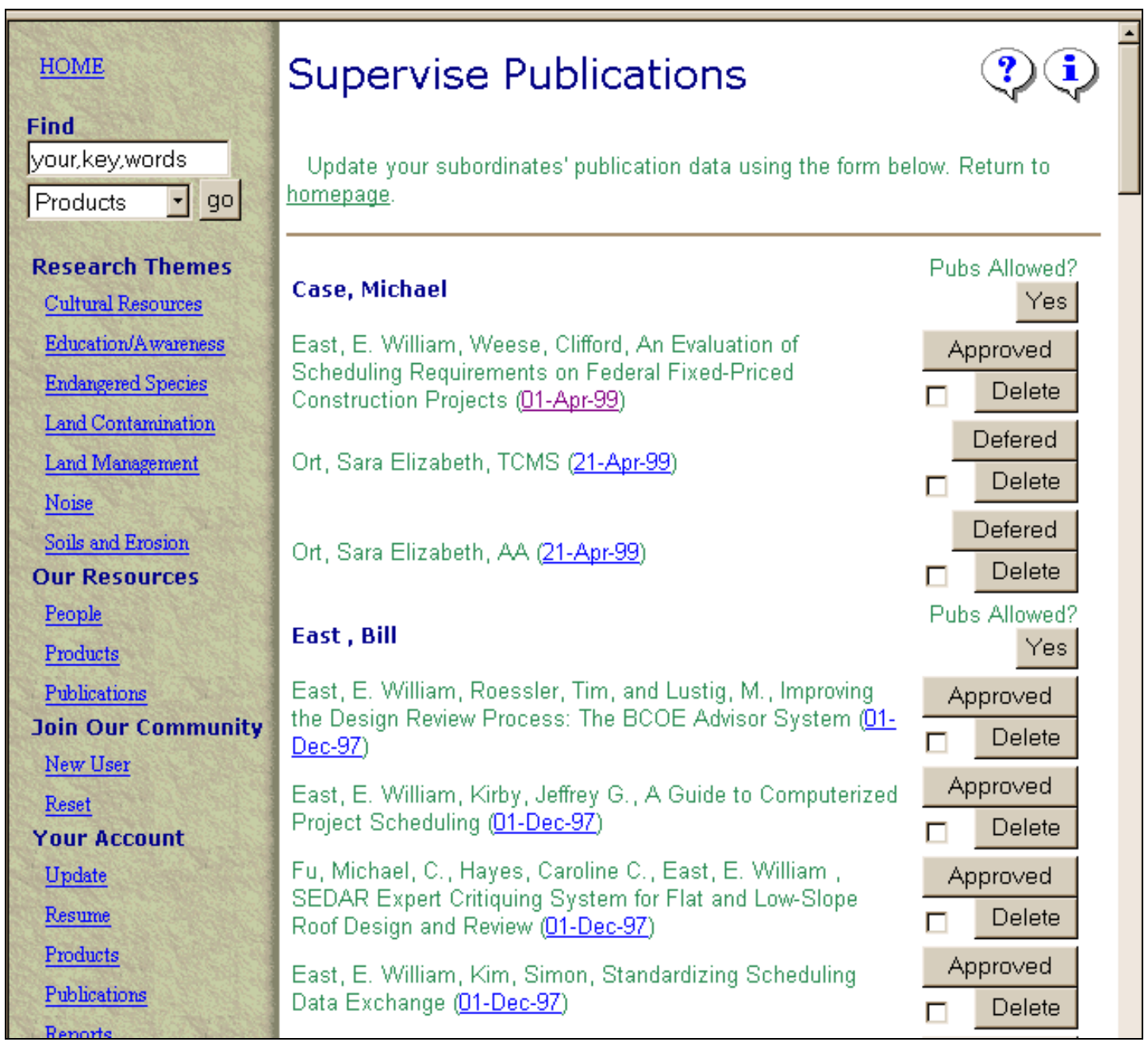

Figure 30. Approve publications. 
A review of the publication may be conducted by clicking on the date on which each publication was updated. After a review of the publication, a supervisor may "Approve" or "Defer" public access to a publication or "Delete" the publication using the buttons to the right of each citation.

\section{Program Manager Process: Add Research Theme}

Program managers may describe the general categories of research conducted in the business area by creating Research Theme Areas. These research themes must be added and subsequently approved before they appear on the TIPS menu. Figure 31 shows the form for adding a research theme.

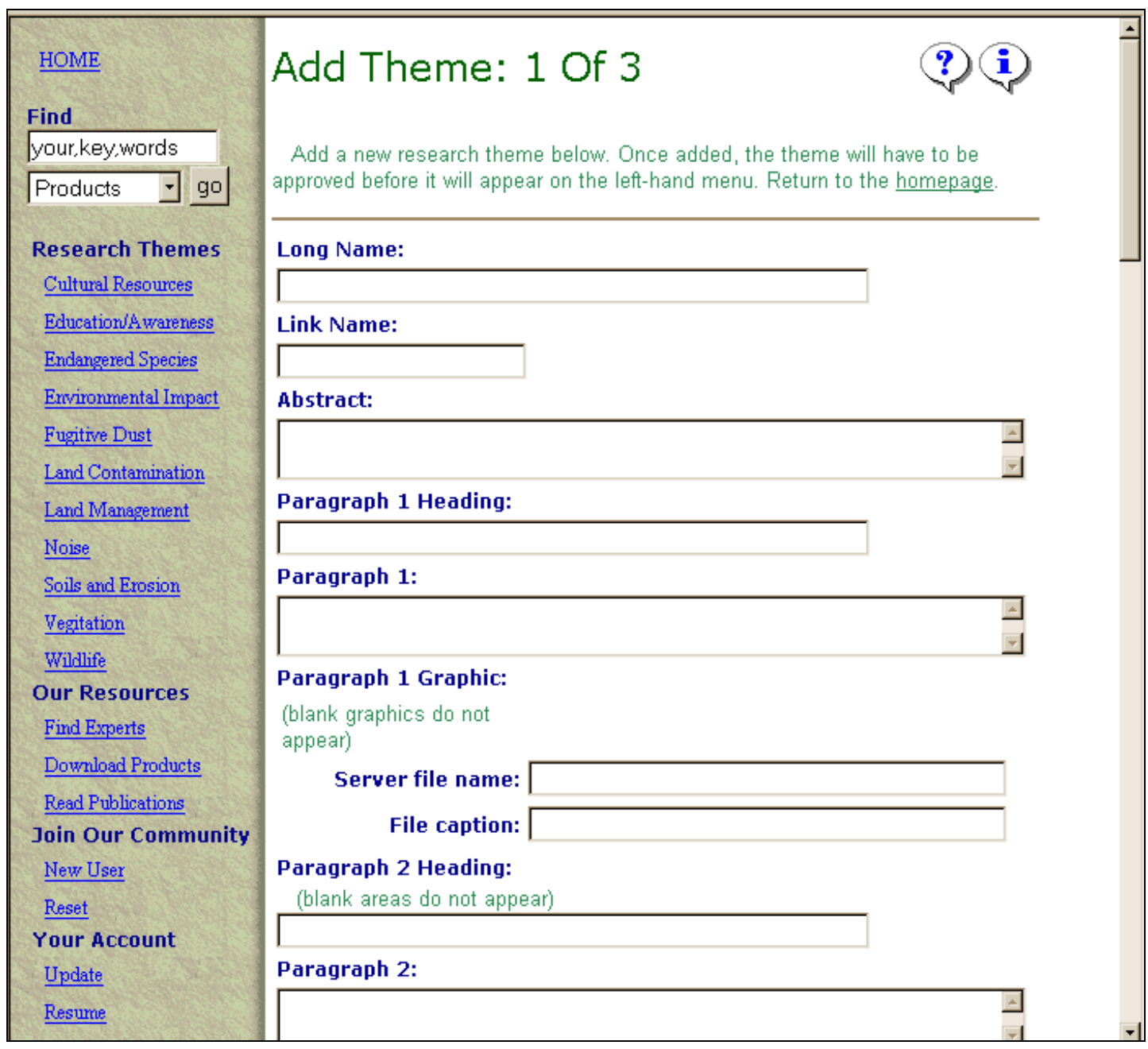

Figure 31. Add research theme. 
The long name is the name that appears on the theme page. The link name is the name that appears in the navigation menu on all pages. The abstract is a one-sentence description of the research area. Following the abstract there are five sets of paragraph heading and paragraph text fields. These fields may be completed, as needed, to describe the theme. The first three themes also allow the project manager to include graphic file names and customize the captions for those file names.

\section{Program Manager Process: Update Research Themes}

After entering research themes, the program manager or site administrator must approve the theme. This process begins with the selection of an existing theme to update from the current list of themes as shown in Figure 32. The themes that have been approved, and therefore appear on the navigation menu, are designated by "(y)." A theme that has not been approved is designated by "(N)."

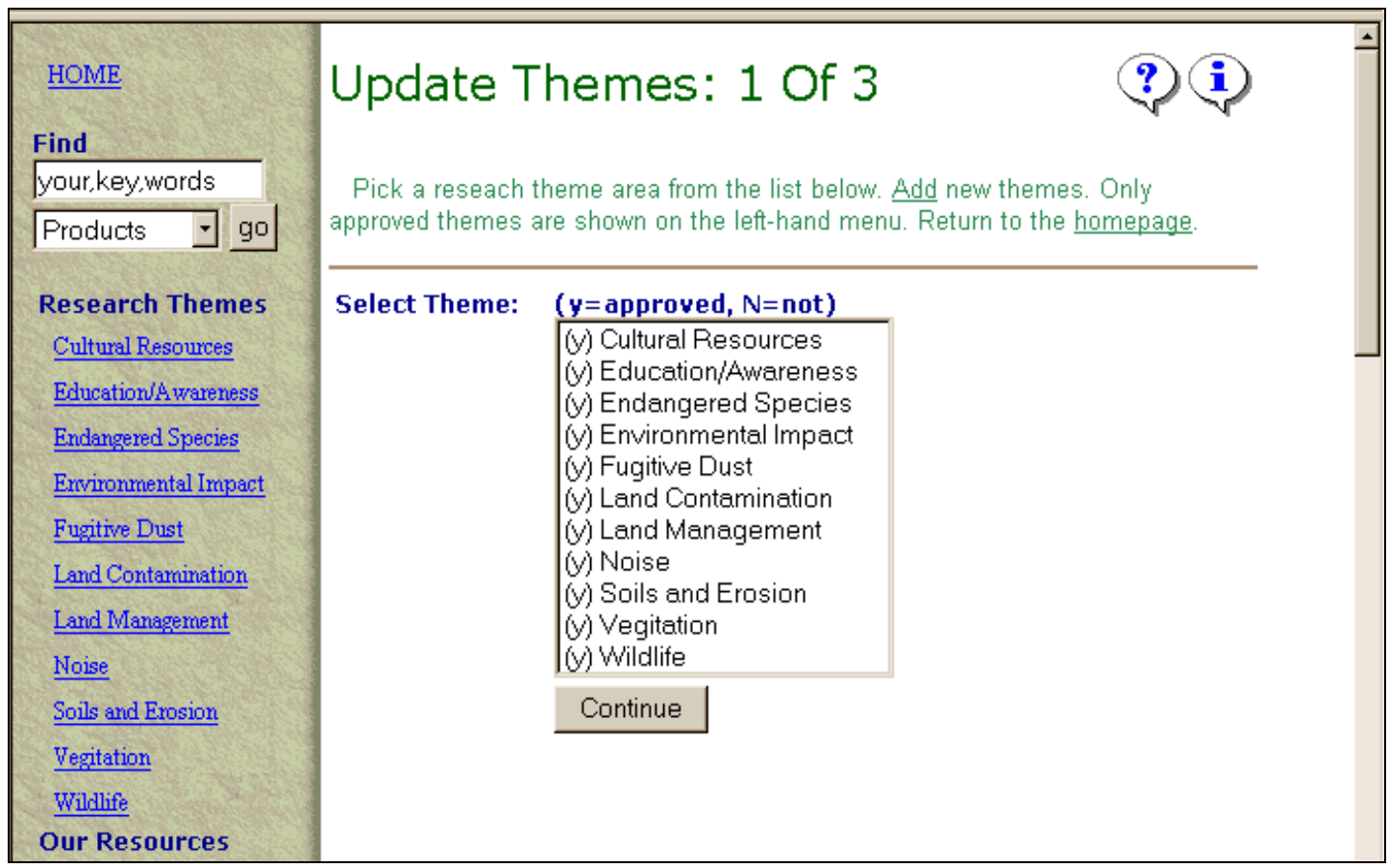

Figure 32. Select existing theme. 
After selecting a theme, the data contained in that theme is provided in an update form. The data may be edited and then updated. After reviewing the data for the research theme, the program manager may either "Save and Continue," "Continue," or "Delete" as shown in Figure 33. If the program manager does not delete the theme, then the next step is to pick those registered users who should be associated with the research theme. A multi-person select list with add and delete buttons is provided for this purpose.

\section{Program Manager Process: Approve Research Themes}

A research theme entry must be approved prior to being viewed by the public. Any program manager (including the original submitter) may approve a research theme. To approve a theme, the theme is first selected, then the form at the bottom of the update page (shown in Figure 33) may be used.

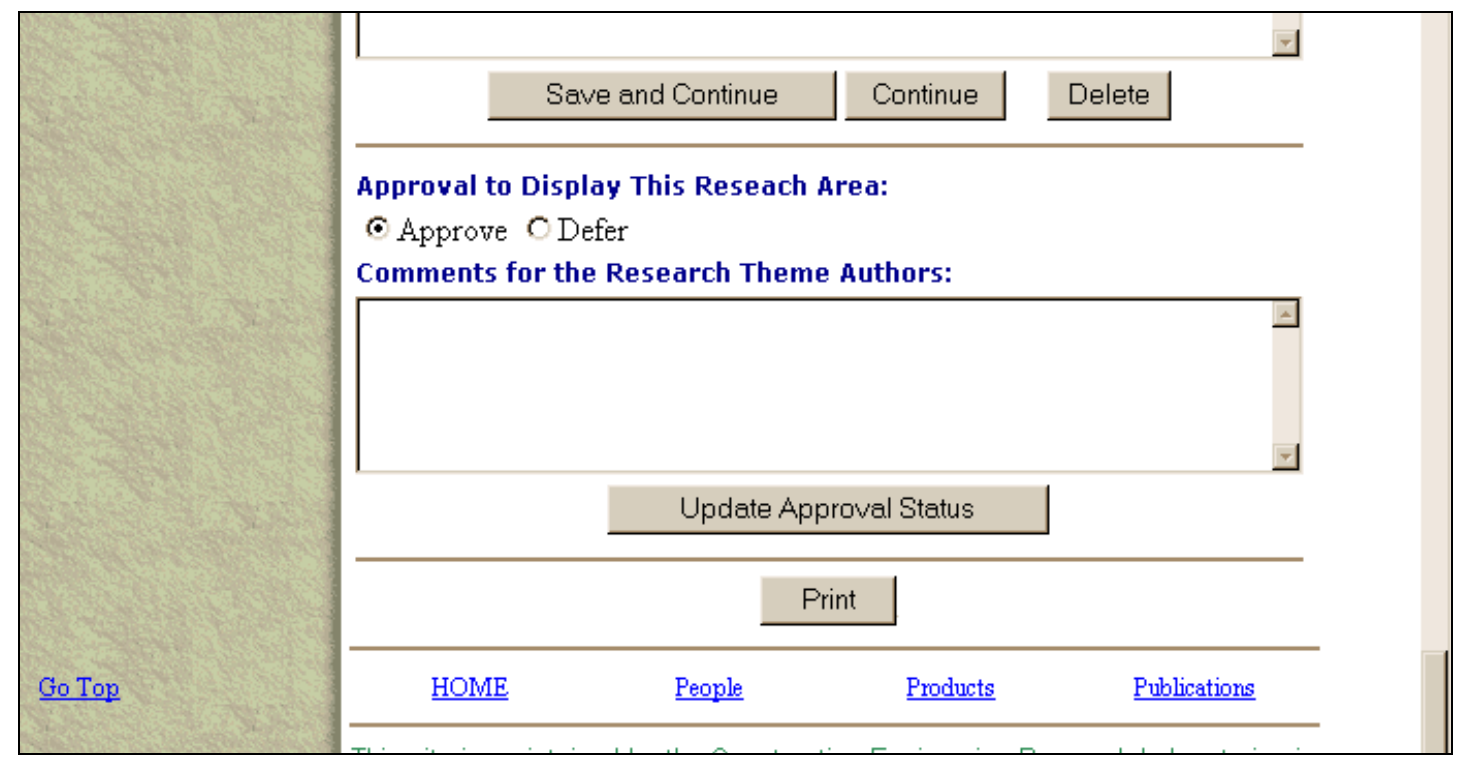

Figure 33. Theme approval. 


\section{Program Manager Process: Designate Hot Products}

Program managers may designate individual products to be highlighted in TIPS. Figure 34 shows the form used to designate "hot products." A list of all products is shown in the left-hand multiple item select box. The right-hand multiple item select box shows the currently selected hot products.

To add one or more new projects to the list, highlight the products in the lefthand select box and click "Add $>$." To remove one or more projects from the "hot list" highlight the products in the right-hand select box and click "<Del."

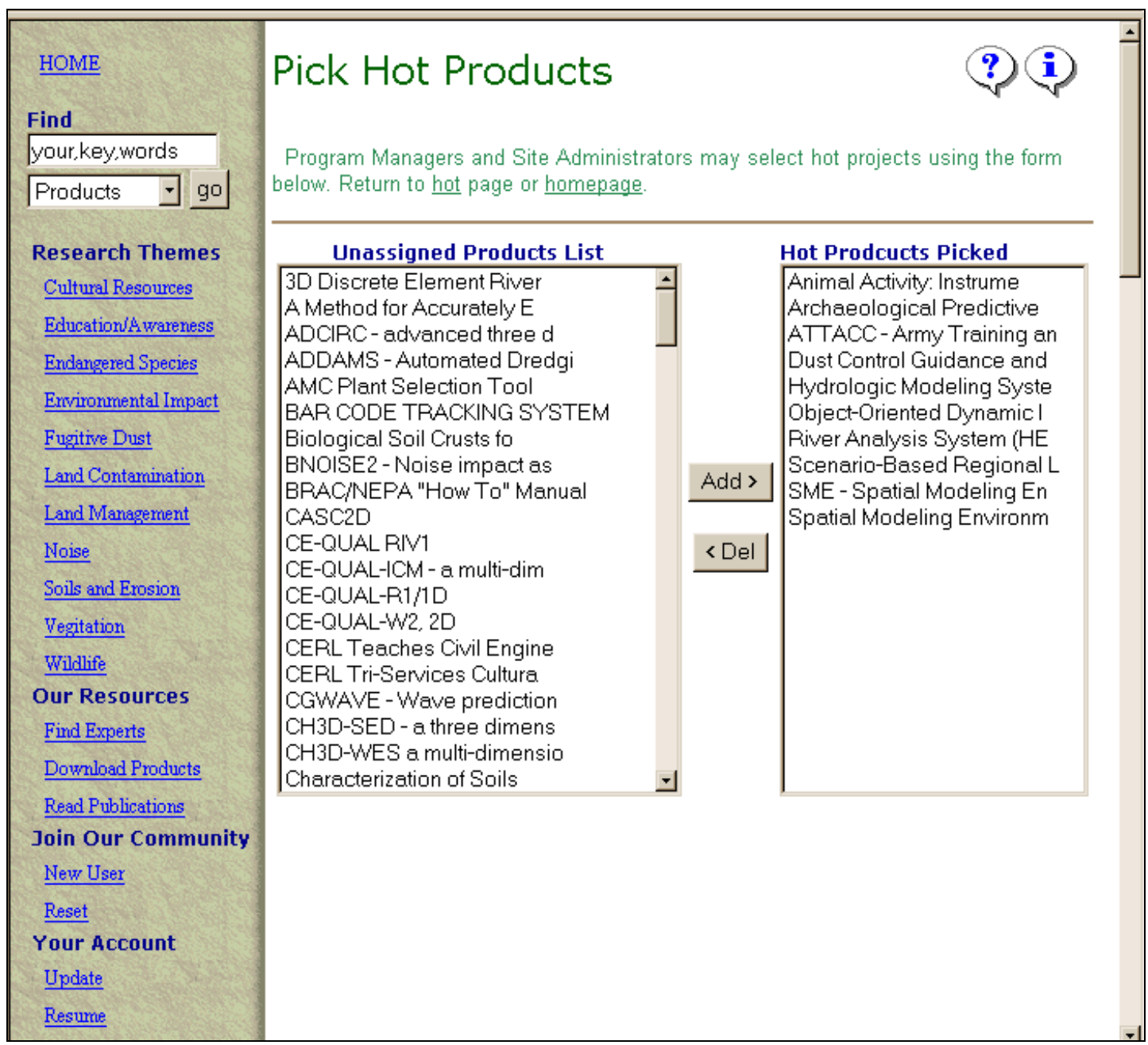

Figure 34. Pick hot products. 


\section{Program Manager Process: Designate Hot Publications}

Program managers may designate individual publications to be highlighted in TIPS. Figure 35 shows the form used to designate "hot publications." The list of all publications is shown in the left-hand multiple item select box. The righthand multiple item select box shows the currently selected hot publications.

To add one or more new publications to the list, highlight the publications in the left-hand select box and click "Add >." To remove one or more publications from the "hot list" highlight the publications in the right-hand select box and click “४Del."

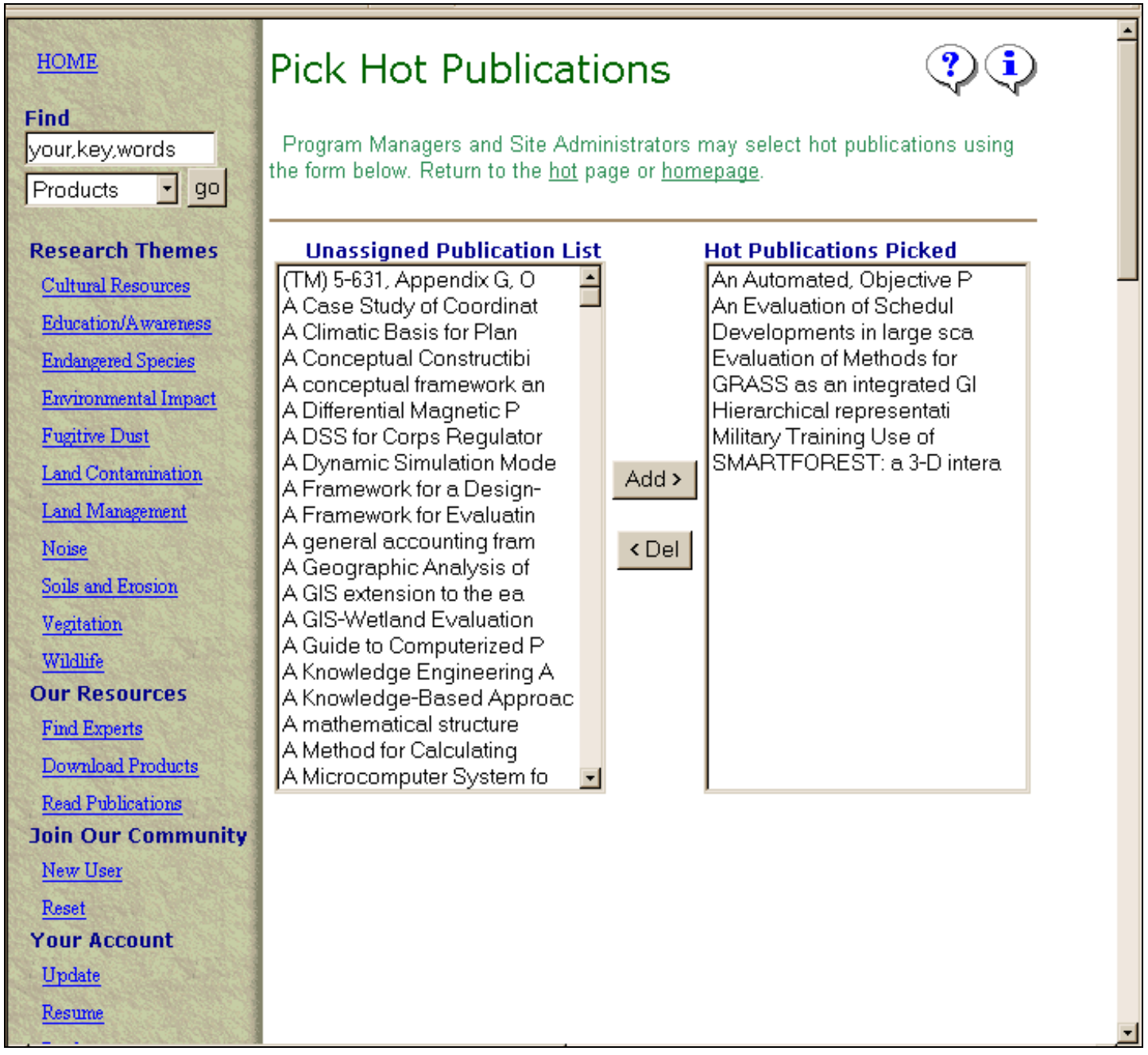

Figure 35. Pick hot publications. 


\section{PAO Officer Process: PAO Fact Sheets}

While the content for a given technical domain may be quite dynamic, with many prototype product descriptions being added and deleted, a subset of these products, capabilities, or general information may also be appropriate for use as official organization "F act Sheets." The purpose of the process described in this section is to allow PAO Officers to take advantage of the updated business area product information that could be used to directly maintain and produce official fact sheets.

The first part of this process begins with the designation of which products might be candidates for PAO Fact Sheets. Figure 36 shows the form that may be used by the PAO Officer to select from the set of all products. When selected, the item is added as an unapproved item member of the set of PAO F act Sheets.

After selecting a product as a potential PAO Fact Sheet, the PAO Officer must review the fact sheets selected. The officer may review one of the selected product descriptions using the form shown in Figure 37.

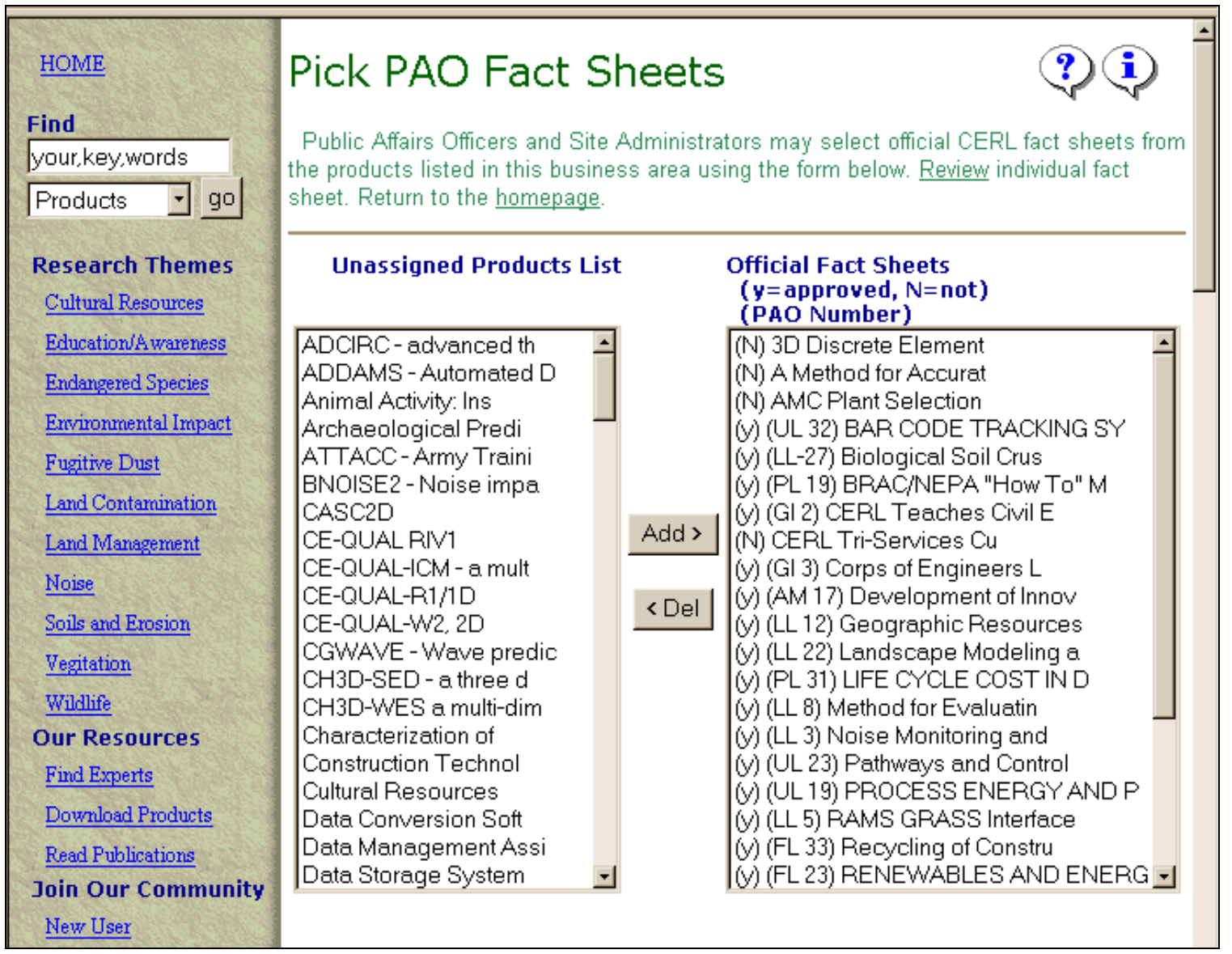

Figure 36. Pick PAO Fact Sheets. 


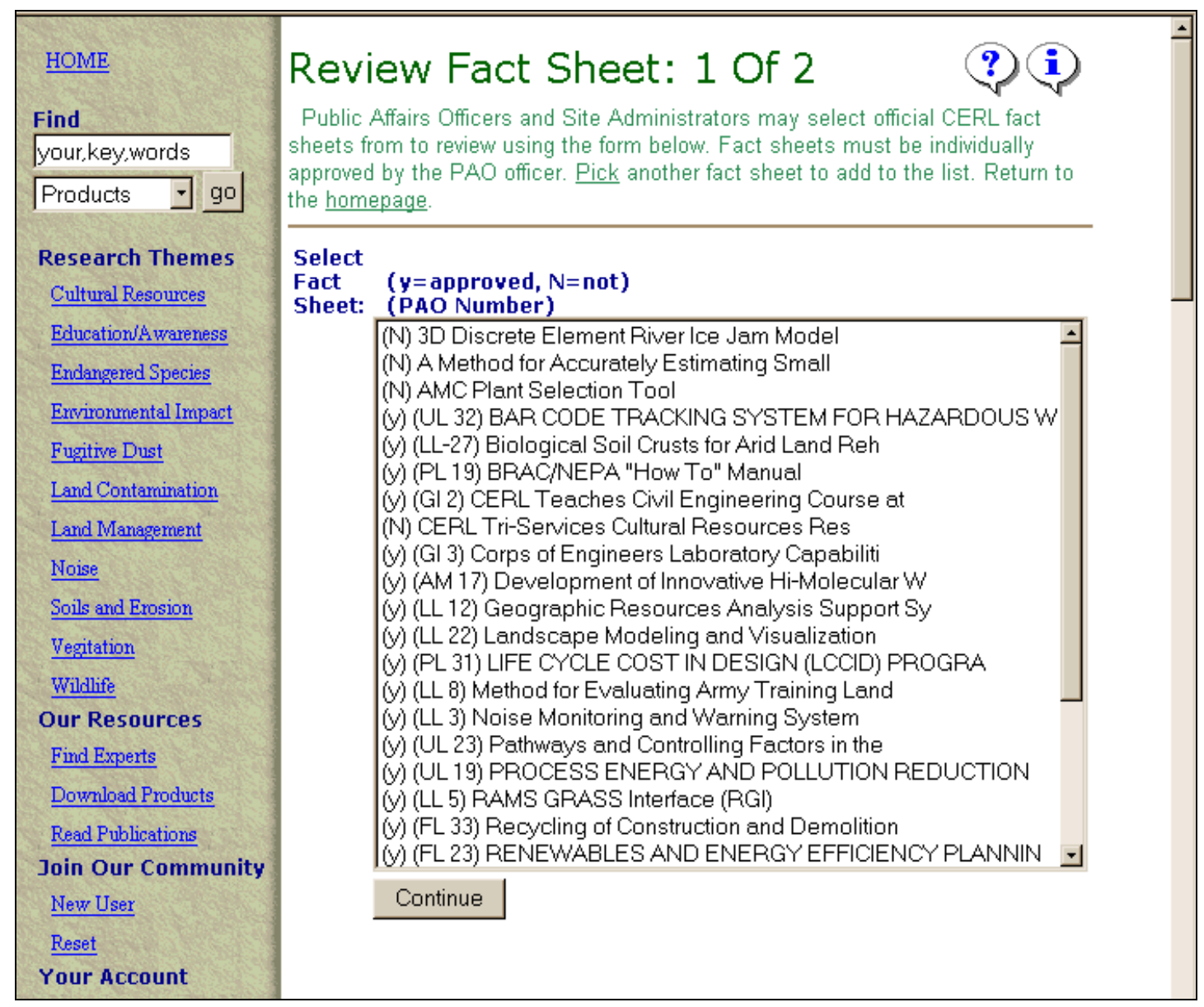

Figure 37. Pick fact sheet to review.

After selecting the product description, the PAO Officer may update the text of the proposed fact sheet directly, as needed, and update the fact sheet. If it is of high enough quality to become an official PAO Fact Sheet, then the PAO Officer may approve the item. As the PAO Officer uses the form shown in Figure 38, automated e-mail is sent to the product POCs. 


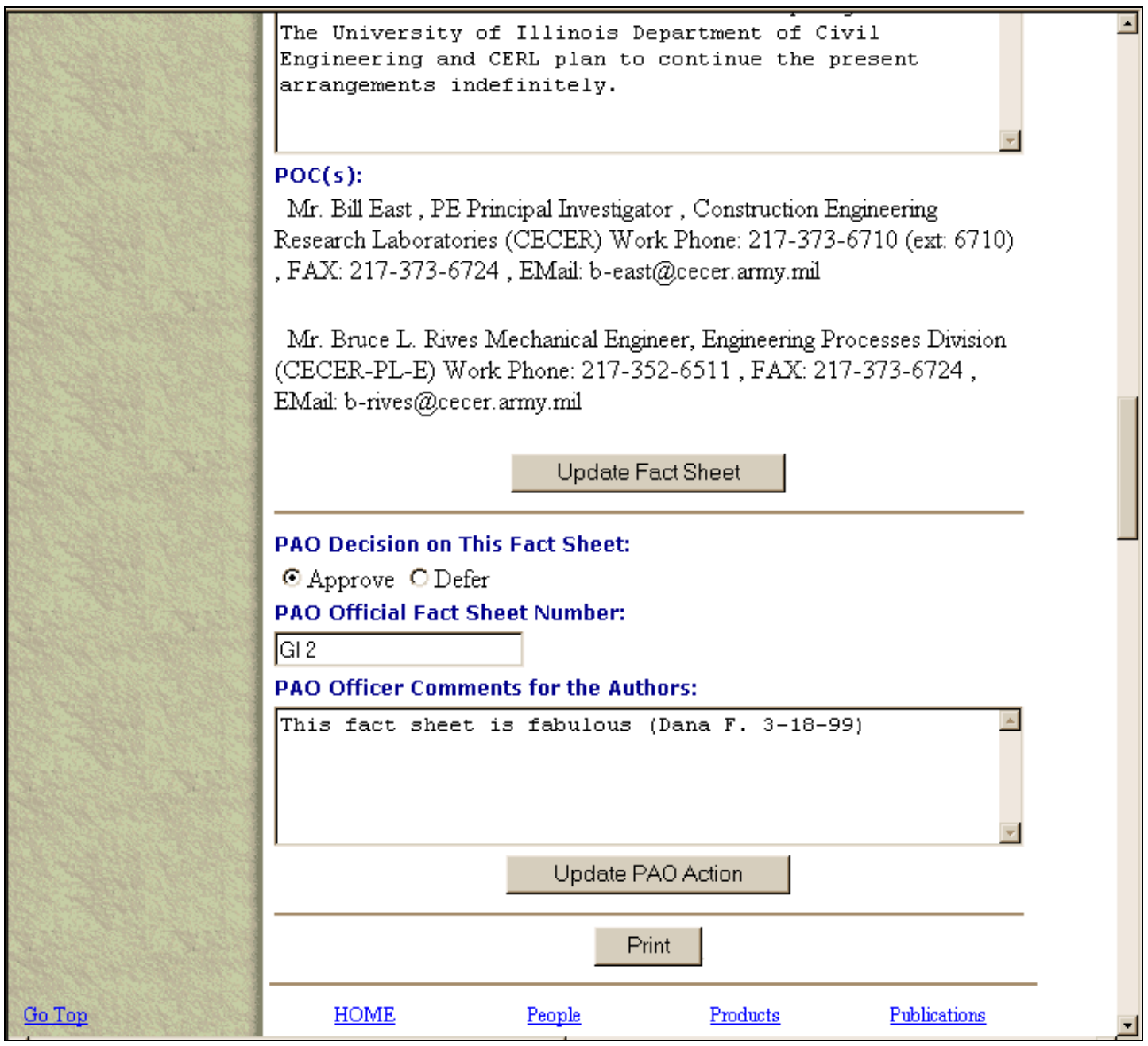

Figure 38. PAO Fact Sheet approval. 


\section{Administrator Process: Update Administrative Settings}

Figure 39 shows the administrative settings that are available to the system administrator. There are five general categories of information: Site Information, Site Appearance, User Administration, Publication Administration, and Resume Administration.

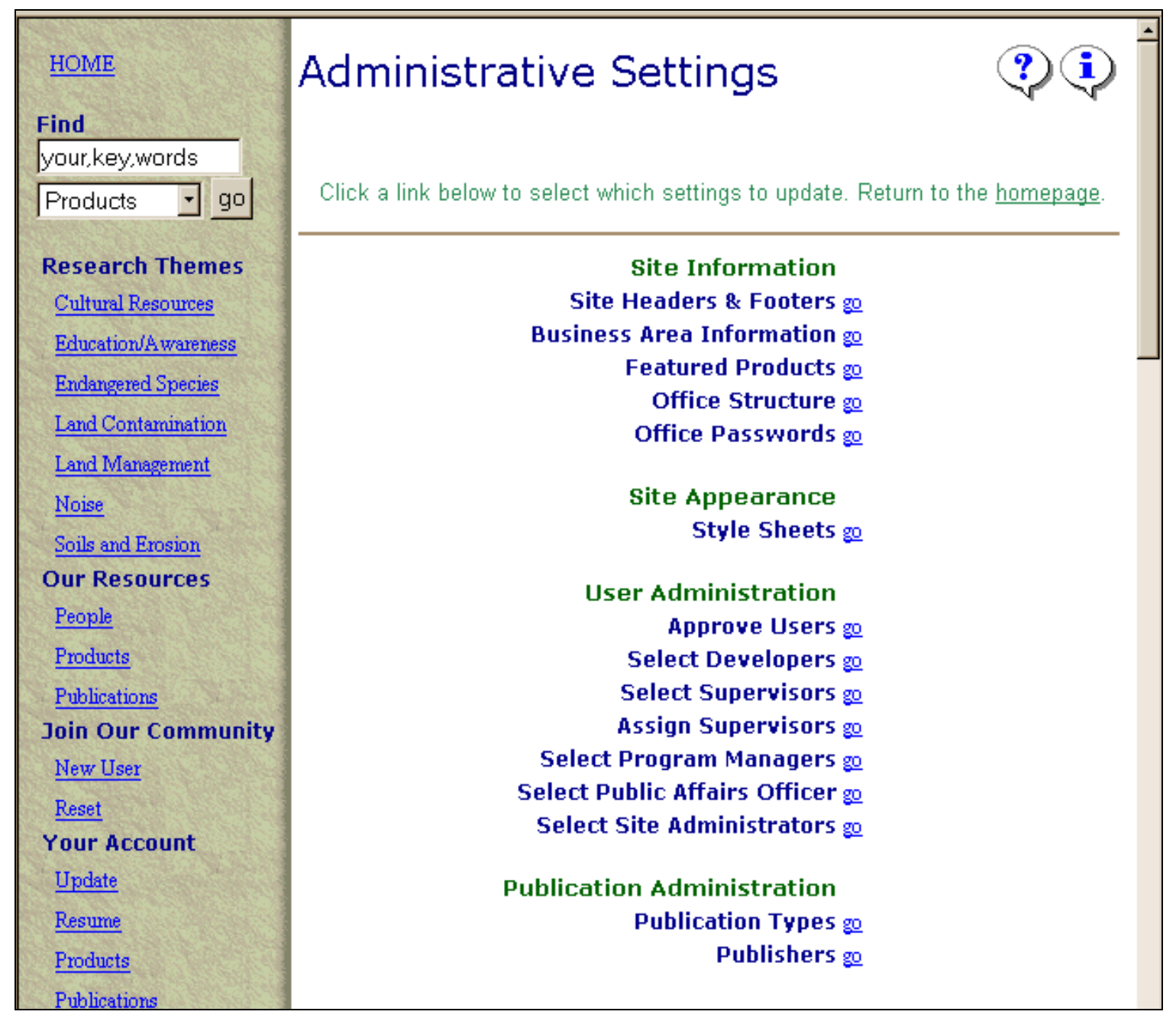

Figure 39. Administrative settings.

\section{Site Information}

Changing the "Site Headers \& Footers" allows the administrator to customize the site for each technical domain. Since all domains may not be contained on the same server, this information also supports future expansion of the program to other ERDC sites. Additional information about the support of multiple domains within TIPS is provided in Chapter 7. 
Updating "Site I nformation" and "F eatured Products" allows users to change the homepage for the current business area. Several graphic files are required for the homepage. These files are for the "What's New," "What's Hot," and three "Featured Products" links. If these files are not provided, the caption will be shown without any graphic link.

Figure 40 shows the page for changing the office organizational structure. The tree structure shown in user registration pages is set up using the "ID" and "Parent" column data. IDs are provided by the system automatically and cannot be changed. To add a multiple-level office, begin by adding the top level for the office and then saving the data. Next add the lower-level offices. This procedure is needed for the system to assign an "ID" to the top-level offices prior to using that ID as the "Parent" in a subordinate-level office.

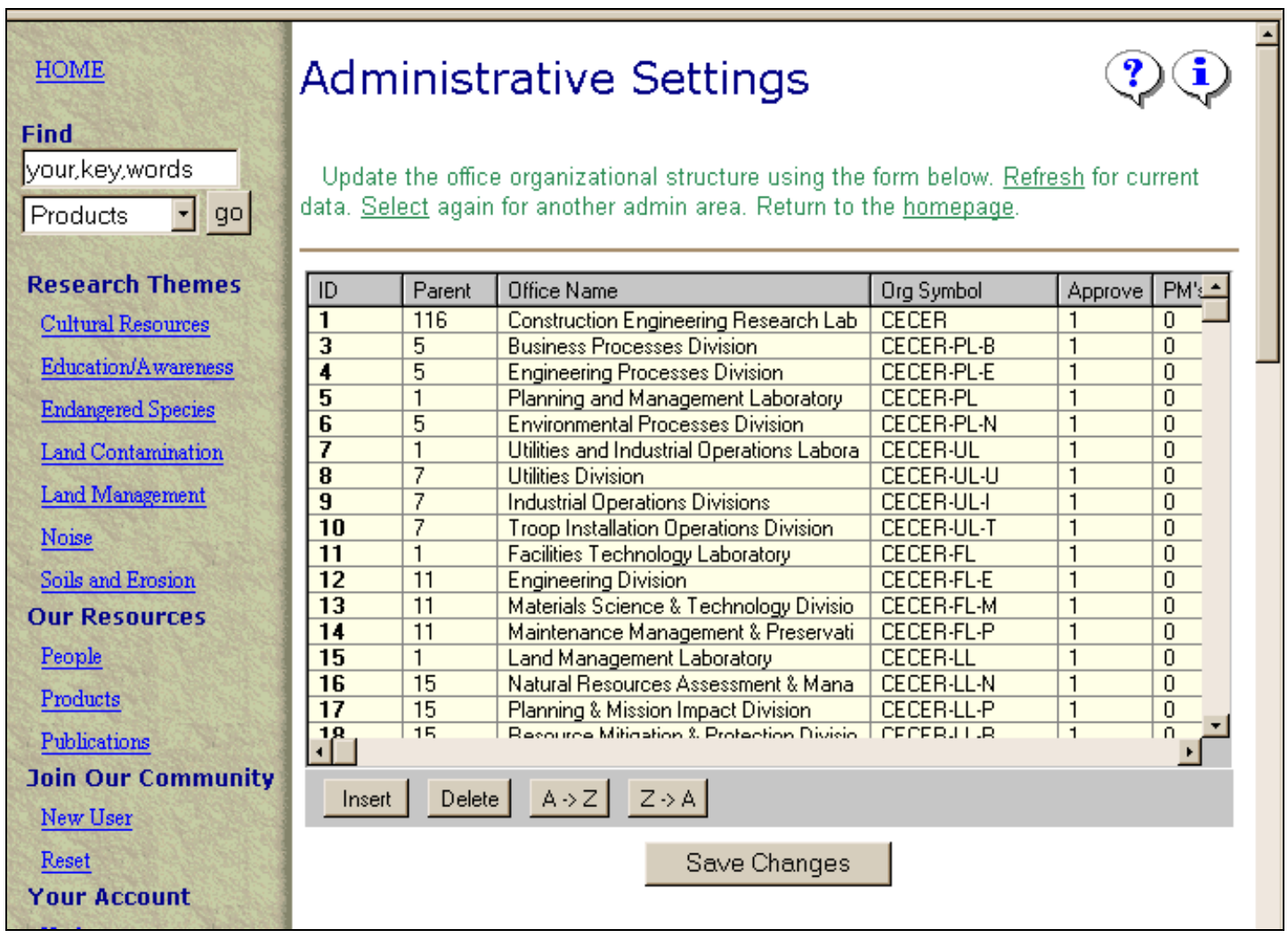

Figure 40. Update office structure.

To the right of the Organizational Symbol column is the "Approve" column. To be visible to users, the organization must have a "1" in the "Approve" column. Useradded offices, entered during the registration process, must be approved for general use by placing a " 1 " in the "Approve" column. The default permissions associated with individuals correctly registering with the appropriate office are shown on the right of the grid. When registering, users will automatically be 
provided the level of permission associated with the office. The last column is the office password (not shown). The new user must use this password when selecting the office during registration.

The system administrator is responsible for maintaining the office passwords. The list of office passwords should not be released. Individuals in a given office should only have access to their specific office's password.

\section{Site Appearance}

As discussed in Chapter 3 under System Administrator, the business area web site uses an HTML (Hypertext Markup Language) technology called Cascading Style Sheets. CSS technology allows the appearance of the web site to be changed in one central location for all pages. Additional information on CSS may be found in a wide variety of on-line sources. Figure 41 shows a portion of the style sheet form. Appendix A contains a detailed description of the suggested contents of each of the CSS classes contained in TIPS.

\section{User Administration}

A variety of user administration options are available to the system administrator. These options include: approving Users, selecting Developers, selecting Program Managers, selecting Supervisors, sel ecting PAO Officers, assigning Supervisors, and designating Site Administrators. All of the pages that process selections appear similar to that for approving users, which is shown in Figure 42. Unapproved users are shown in the left-hand multiple selection box. To approve users, the administrator selects one or more names from the left hand box, then clicks the "Add >" button.

When users are approved, both the user approval and allow reports options are turned on for each user account. When the developer selection is conducted, the "Devel oper" tag is turned on for the selected accounts. A similar procedure turns on the user account tags for Program Managers, Supervisors, PAO Officers, and Administrators. Assignment of supervisors will support a future designation of alternate supervisors, from other organizational el ements. 


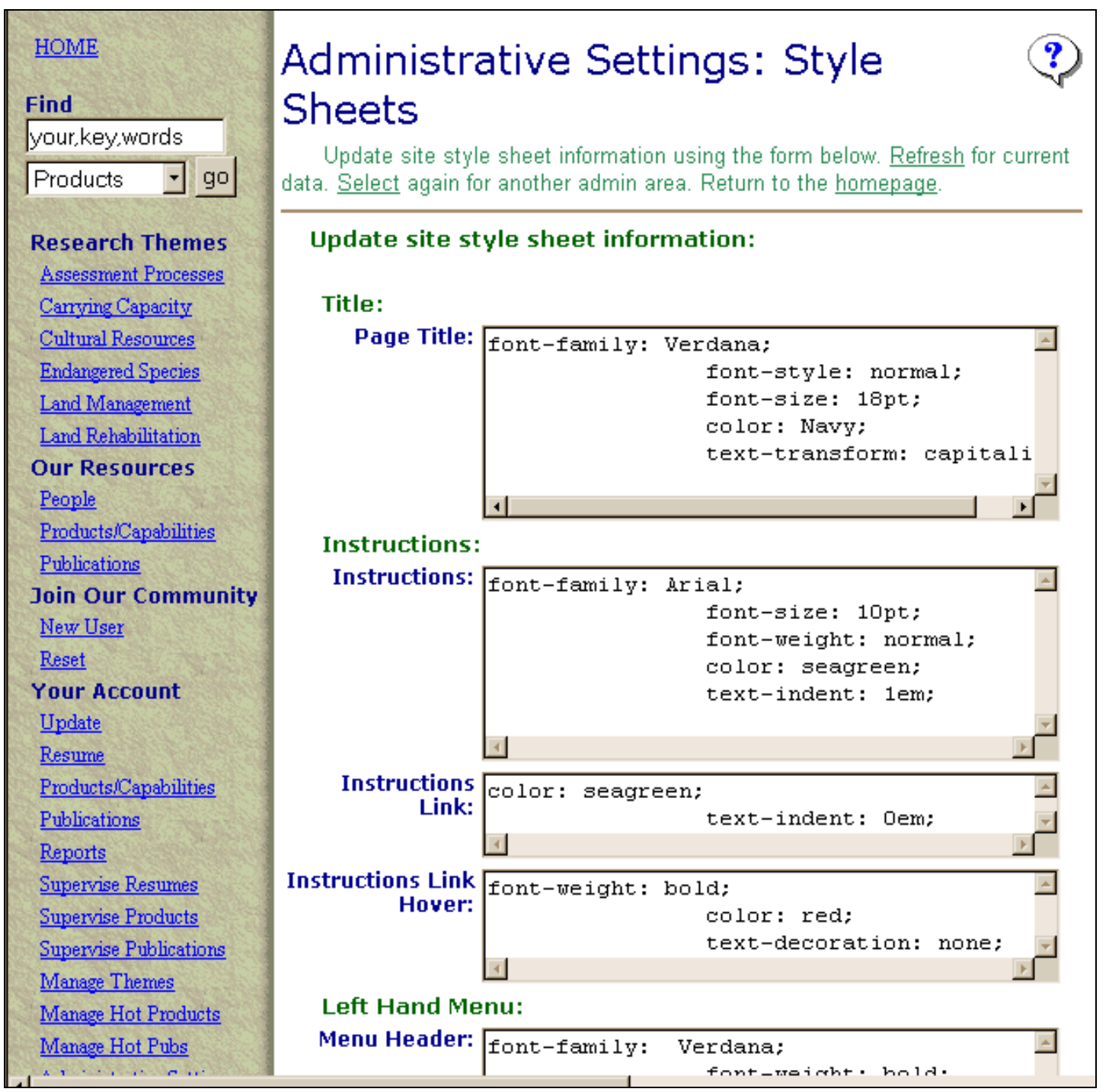

Figure 41. Update style sheet.

\section{Publication Administration}

Administration of publications allows administrators to change two types of pick lists related to publications: Publication Type and Publishers. This information is displayed to the administrator in a grid that can be updated with all changes and then saved.

\section{Resume Administration}

Options to administer publications include changing pick lists for various types of awards, degrees, professional registrations, and society affiliations. Each of these sets of information is displayed in a grid that can be updated with all changes and then saved. 


\begin{tabular}{|c|c|c|c|c|}
\hline $\begin{array}{l}\text { HOME } \\
\text { Find } \\
\text { your,key,words } \\
\text { Products go gol }\end{array}$ & \multicolumn{4}{|c|}{$\begin{array}{l}\text { Administrative Settings: } \\
\text { Approve Users } \\
\text { Update the list of approved users using the form below. Refresh for current } \\
\text { data. Select again for another admin area. Return to the homepage. }\end{array}$} \\
\hline $\begin{array}{l}\text { Research Themes } \\
\text { Assessment Processes } \\
\text { Carrying Capacity } \\
\text { Cultural Resources } \\
\text { Endangered Species } \\
\text { Land Management } \\
\text { Land Rehabilitation } \\
\text { Our Resources } \\
\text { People } \\
\text { ProductsiCapabilities } \\
\text { Publications } \\
\text { Join Our Community } \\
\text { New User } \\
\text { Reset } \\
\text { Your Account } \\
\text { Update } \\
\text { Resume } \\
\text { ProductsiCapabilities } \\
\text { Publications } \\
\text { Reports } \\
\text { Supervise Resumes }\end{array}$ & $\begin{array}{c}\text { All Unapproved Users } \\
\qquad \text { Wienke, Gloria }\end{array}$ & Add > & $\begin{array}{l}\text { All Approved Use } \\
\begin{array}{|l}\text { Anderson, Alan } \\
\text { Athow, Bob } \\
\text { Balbach, Harold } \\
\text { Bandy, John } \\
\text { Black, P. } \\
\text { Boyt, Bill } \\
\text { Bruzewicz, Andrew } \\
\text { Butler, Cary } \\
\text { Case, Michael } \\
\text { Chawla, Manroop } \\
\text { Collins, Nicholas } \\
\text { Corbin, Calvin } \\
\text { Croisant, William } \\
\text { Dale, Virgina } \\
\text { Daly, Steven } \\
\text { D'Angelo, Vincent } \\
\text { Davo, Theresa } \\
\text { Deliman, Patrick } \\
\text { Denight, Michael } \\
\text { Dilks, Kelly }\end{array}\end{array}$ & \\
\hline
\end{tabular}

Figure 42. Update approved users. 


\section{Creating a New Technical Domain Area}

TIPS was designed to take advantage of the distributed nature of the Internet. In this distributed configuration, technical domains may be located on web servers under the control of those offices where the technical domains are managed. While such a configuration may not, ultimately, be selected under the proposed ERDC-wide TIPS system, this section of the report describes how to install the current version of TIPS on new servers based on the original distributed configuration.

Two overall phases of work are required to create a new technical domain using the generic site developed under this project. The first phase is to create a working copy of the generic TIPS site at the location where the new domain is to be developed. Following that, the new generic site will need to be customized with information about that new domain. The majority of the customization tasks required for a given technical area will be the same regardless of the future centralized TIPS configuration.

\section{Minimum Server Requirements}

Required server software for TIPS is either N etscape Enterprise Server or Internet Information Server. Separate directories for data and web pages should be created to ensure that data files are only accessible through the application server, Cold Fusion ${ }^{\circledR}$. Server hardware may be purchased from any reputable vendor, such as those on the U.S. General Services Administration (GSA) schedule. In a distributed configuration, TIPS may share use of the Cold Fusion ${ }^{\circledR}$ application server with many other applications on the same web server hardware.

\section{Configuring the Server}

Begin by allowing read, write, and execute permissions to the person(s) creating the new web site for the web server directory above which the site will be created. It is recommended that permission to the web page root directory access be provided so that only one directory name will be in the "http://office.org.domain/ directory/" path to the web site. Following creation of the site, the root directory 
write access may be turned off. Read, write, and execute permissions will continue to be needed for developers in the top-level site directory and all subdirectories. All other users will require read and execute permissions on the toplevel site directory and all subdirectories.

Any directory may be used for data files, as long as data files are not under the web page root directory. In general, an "ht-data" directory is created under which all data files used by any web pages are located.

When appropriate, the web server administrator may provide a shared network drive to development personnel to allow them to remotely access the web programs, documents, and data files directly. Of course, passwords on such shared drives should be very robust if used across the Internet.

As a note to the web server administrator, the server directory where the new site is to be located should not be password protected, nor should the server be password protected. The TIPS site does not integrate with LDAP (Lightweight Directory Access Protocol) or other server-based password protection schemes. Also, encryption of web server data or program pages was not considered to be necessary for this application.

The web server administrator should begin the process by copying all the pages of a generic TIPS site to the new location on the web server. Copying the entire directory should be the easiest way to proceed. Several types of files will be copied, including: web pages (*.htm, *.html), Cold Fusion ${ }^{\circledR}$ web page templates (*.cfm), and graphics files (*.gif, *.jpg). Data contained in MS Access ${ }^{\mathrm{TM}}$ data files (*.mdb) also needs to be duplicated at the new database directory.

Below the name of the top-level directory, subordinate directory structures must be maintained exactly as they are provided in the generic TIPS site. Maintaining the subdirectory structure of the site is critical to site operations because the links among pages use relative path names.

Before the site can begin its transition from a generic package to a technical domain-specific application, the web server administrator must complete a number of tasks. First, the database file for the generic web site should be renamed to reflect the new domain area. Second, the database must be registered with the Cold Fusion ${ }^{\circledast}$ application server as a data source. The data source name used in Cold F usion ${ }^{\circledR}$ must also be representative of the technical area.

Next, any absolute path names contained in the generic package need to be changed to reflect the absolute path for the current business area. A package 
such as Cold Fusion ${ }^{\circledR}$ Studio should be able to perform a recursive find and re placement operation across all site subdirectories. A scan of the "index.cfm" file in the root directory of TIPS will reveal the absolute path names that need to be modified.

Next, all Cold F usion ${ }^{\circledR}$ template files (*.cfm) will need to be edited to change the name of the Cold F usion ${ }^{\circledR}$ data source from the name of the generic site to that created for the new site. Again a package such as Cold F usion ${ }^{\circledR}$ Studio should be able to perform a recursive find and replacement operation across all site subdirectories.

Finally the web server administrator needs to create at least one technical domain "Administrator" account and provide a password for that account. Administrators should also be assigned all other access levels so that they can test any system feature before allowing general use.

Adding one or more "Administrators" is accomplished by adding a record to the "Personnel" table of the database. The passwords entered into this table should be sufficient to resist attacks (i.e., nine characters using both letters and numbers).

Once the web server administrator provides each Administrator with a password, those persons may begin to customize the site for their specific business area. Administrators may use the Account | Reset left-hand menu selection to obtain their site security key.

The Administrator should begin by changing the name of the web site and providing appropriate front matter for the new technical area. This may be accomplished using the "Site Headers and Footers" in the "Administrative Settings" menu selection. Site header information includes the name of the domain as well as the telephone number for site support. Unless a specific support staff or contractor has been identified, the "Administrator" should place his/her name in the footer page.

After changing the site header and footer information, the "Administrator" will need to create the office structure for all offices expected to participate in the site. The procedure to create offices is provided in Developer Process: New Office in Chapter 4. Again, passwords should be carefully selected during this process.

After creating the set of offices, site "Supervisors" should register and be approved, by the "Administrator." Finally "Developers" may register and be 
approved. After users are registered and approved, they may begin to add their resumes, products, and publications as described earlier in this report. Supervisory approvals of resumes, products, and publications submitted are then required to allow public access to the information provided. 


\section{User Support}

Support for traditional client/server systems involves an extensive network of services to ensure that thick-client software written in Visual Basic, C, or other programming language is installed and used correctly on each user's equipment. A web-based client/server system significantly reduces the cost of the overall programming effort and also reduces the cost of user support. This is because anyone accessing, for example, TIPS only needs to know how to use free, commercially produced, client software. For TIPS, any version 4.0 or better browser (Explorer or Navigator preferred) will suffice.

Three types of user support are needed for web-based client server systems: help for the general public, technical support to enable user interaction, and support for user feedback. Help for the general public is primarily to assist users in finding information they need. If the user cannot find the information they expect, then a toll-free telephone number should be provided.

The second type of technical support enables users to interact with the application to find out additional information. Two types of page-specific interactions are available in TIPS. The first is a "get more information" icon. When available, the "I" icon appears in the upper right hand corner of a TIPS page. Clicking the " $\mid$ " will bring up a new window where users may ask that the author(s) of the information contact them. The second type of page-specific interactions available in TIPS is a "help" icon. When available, the help icon appears as a "?" in the upper right-hand corner of a TIPS page. On-line help provides users with information on how to use the items found on the current page.

The third type of TIPS support allows users both to provide feedback on page or system design and business processes, and to report page errors. In TIPS, an electronic discussion group has been created to capture, route, and document requests for changes, suggestions, and "bug" reports. This discussion group allows users to see the items submitted by others and serves as the mechanism to track the completion of all requests.

Since TIPS will serve as both the internal system for managing specific business processes and the external viewer for ERDC business resources, it is essential that effective user support services be developed and maintained. The three 
types of services - toll-free support hotline, context-sensitive help, and issue tracking - are essential if TIPS is to be successful. 


\section{Project Management Plan and Evaluation}

While the content of TIPS is limited to the business processes described previously in this report, TIPS itself was conceived within a broader context of organizational corporate data management. This context includes project data, reports to customers, financial data, personnel data - essentially all data necessary for organizational operations and the means by which all these various data interact.

TIPS contains only a subset of ERDC corporate data. Many other systems are in place for these other types of organizational data. TIPS will be able to draw from these systems where possible and appropriate to ensure synchronization among corporate data stores and minimization of data entry and maintenance requirements.

The overall TIPS concept shown in Figure 43 provides the maximum effective use of corporate knowledge. Many different systems can be woven together to achieve this purpose, and these systems will change and evolve as organizational processes and underlying information and communication technologies change and evolve. I mplementation of TIPS will be most effective in organizations that have a similar broad conceptual framework for corporate data management and use.

\section{Phase I and II: Development of an Operational Prototype}

The development of TIPS has been designed in phases. Success of the next phase depends on the successful implementation of the previous phase. At each phase more features and users are added as the scope of the effort increases. TIPS, Phase I was completed for a single technical domain, Conservation Technology, in FY98. The goal of Phase I was to determine if web-based business process systems could simultaneously support the internal and external customer needs. Phase I successfully demonstrated that, if corporate data were captured as researchers' performed their normal business activities, then the data could also be used for management reporting and customer outreach activities. 


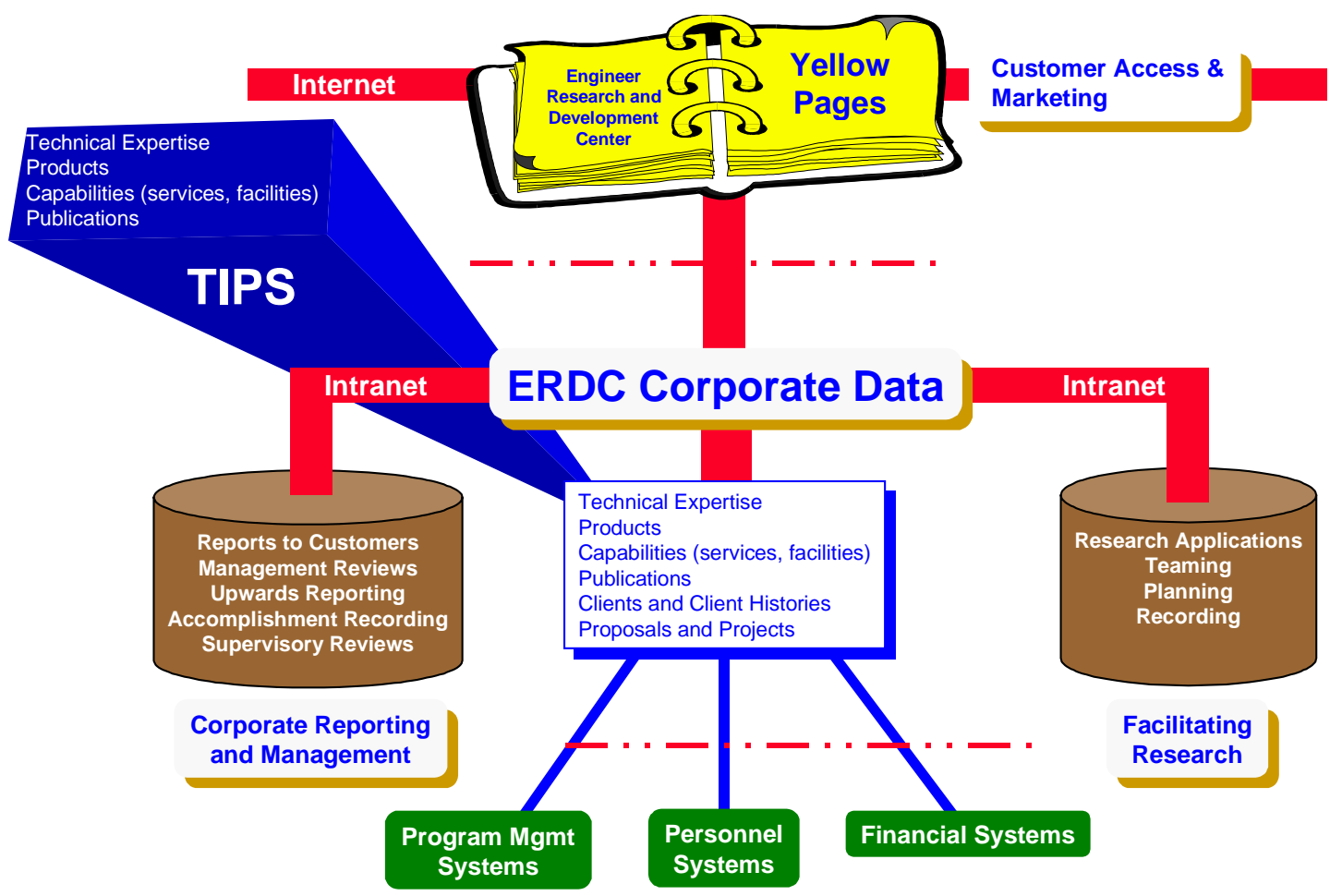

Figure 43. Overall TIPS concept.

Sites for three technical domains (Military Land Management, Installation Operations, and Facility Acquisition and Revitalization) were created and implemented during Phase II. Features to ensure management review, capture specific business processes, including automated e-mailing, and others were added during Phase II.

During Phase II, a more formal advisory committee was established to validate requirements for the development effort and provide organizational planning for TIPS implementation at CERL. The committee reviewed ERDC and Corps of Engineers guidance on web sites and management information systems to ensure compliance. This committee also engaged parties from across ERDC to evaluate TIPS to determine its applicability to ERDC. Coordination with the ERDC Management Information System (MIS) Committee also ensured that TIPS was not duplicating efforts underway at other ERDC sites.

\section{Phase II: Resources}

The Phase II planning team spent approximately 4 calendar months working these design issues and drafting initial Corps Life Cycle MIS (LCMIS) documentation before beginning Phase II programming. Expenditures during this 4month period included 3 analyst-months and 2 manager-months. 
Once the planning period was completed, computer coding to move from Phase I to Phase II required 4 calendar months, 1 analyst-month, and 3 programmermonths. As of J uly 99, TIPS Phase II was ready for populating with updated information from the Military Land Management domain and new data from the Installation Operations and the Facility Acquisition and Revitalization research domains.

At the conclusion of FY99, it is expected that TIPS for the three technical domains that primarily operate at the ERDC Champaign campus will be operational.

\section{Phase II Evaluation: Opportunities and Challenges}

TIPS presents both a great organizational opportunity and challenge. The opportunity is for everyone who connects with ERDC, in any capacity, to have an easy reference source to who and what ERDC is and does. The challenge is to effectively engage all the investigators within ERDC in populating and updating this knowledge base. Meeting this opportunity will give ERDC an outstanding competitive advantage - ease, accuracy, and consistency in obtaining information are important criteria for any customer when making a decision about where and with whom to do business. There are excellent web sites scattered across ERDC already, but they are limited to specific technical units or products and do not bring all of ERDC's capabilities and products to ERDC customers, partners, and workforce. TIPS promises to become the ERDC yellow pages, with the added power of information and communication technology advancements.

Because of the remarkable growth of the web, TIPS-like capabilities are rapidly emerging and will become commonplace in the near future. Customers will soon expect organizations to provide web searchable product and capability information. All of the necessary software and networking functionality is currently in place for TIPS, and will no doubt rapidly improve in performance and throughput capabilities.

The difficulties in making TIPS successful are not so much technical as organizational. The Phase I and II approaches to TIPS depend on the data input of the technical workforce, which will allow the real experts to be proactively providing and managing (with organizational review) the information about their technical work. The opportunity presented by TIPS will not be fully realized, however, unless the difficulties of populating and maintaining the data fields are adequately addressed. Too often, organizations make an initial push for a new initiative, then shift focus to new projects. If putting data into TIPS is "another 
data drill" that does not replace other drills, then both initial populating and updating of TIPS will be an uphill struggle, requiring strong-arm management measures to enforce.

The value of TIPS needs to be understood and realized by the workforce that will provide the data. Customers browsing TIPS and sending e-mail to listed POCs will be an essential selling point for the workforce to stay enthusiastic about spending time updating TIPS. Nurturing customer use of TIPS will be an important responsibility of all members of the workforce.

In addition, management use of TIPS for regular business reports and analysis will be necessary to keep the workforce engaged in populating and updating TIPS over the long term. TIPS needs to become the sole avenue for all technical document traffic inside the organization, the primary internal and external source for all web-based product information, and the source of many elements that enter into organizational reports and evaluations. For example, TIPS should be used for all sorts of analysis, such as:

- How many and what kinds of technical reports were published throughout the organization (or within a sector of the organization) over the last fiscal year?

- What is the trend in product types over the last 5 years?

- Which customers use TIPS on a regular basis, and how often did the use of TIPS result in new projects?

- What is the status of my approval for release of a technical paper?

- How many technical documents are currently in the approval/editing pipeline, and what is the average load, and how does this current load compare?

- What persons across the organization have previous work experience in Central or South America?

- What products, capabilities, and expertise in the organization might be relevant for the new Department of Transportation "rebuild America's railroads" or the new EPA "export environmental technology" programs?

The committees that plan for organizational implementation of TIPS will need to tightly define many of these analysis/reporting options, and proactively make the 
organizational adjustments to shift processes and information flow through the TIPS environment.

Given the opportunities provided by TIPS Phase II, working toward a TIPS Phase III that may provide even more value with reduced organizational cost and minimized personnel disruption seems a feasible goal. The next section highlights the current directions for the next generation TIPS system. TIPS Phase III addresses two essential design concerns in the TIPS II system and incorporates additional cost saving features.

\section{Phase III: IntraCampus Integration}

Initially, TIPS was conceived as a tool to support internal and external exchange of information for researchers in a single technical domain. Such a basic design idea is in keeping with the distributed nature of the Internet and would assist individual technical domain experts in maintaining complete control over their own computer hardware and software assets. As efforts to implement TIPS across more than one technical domain began in Phase II, two questions began to arise. The answers to these questions are currently shaping plans for TIPS Phase III.

The first question is, "How will individuals who participate in multiple technical domains enter data or search across these multiple domains?" The second question is, "Can TIPS capture information needed to support campus-specific web sites?" The preliminary work under TIPS Phase III will resolve these two questions.

In Phase III the distributed database design question will be resolved by moving the Phase II operational prototype system to a more robust database platform. This more robust platform will allow all technical domain areas to be served from a single database. Once information regarding a technical domain is in a single physical location, adding, updating, and searching the set of ERDC technical domains requires less coordination than would have been required with a distributed database application.

The answer to the first question, "How will individuals who participate in multiple technical domains enter data or search across these multiple domains?" is that the technical domain will be a selection made by the user. Users may select one or more technical domains for themselves, their products, or their publications. The new data will default to a user's single, primary technical domain, but the user will be allowed to select from more than one domain. 
The technical solution to this multiple-domain selection is to add a new database table called "technical domain" to the TIPS database. All people, products, and publications will have new linking tables that identify the relationships between the domain(s) and the people, products, and publications.

Regarding the second question, "Can TIPS capture information needed to support campus-specific web sites?", the answer is yes. The majority of a campusspecific web site relates to the technical content of the work performed at that physical location. Additional campus-specific information, such as historical information, general capabilities, community information, etc., may be captured in the same people, products/capabilities, and publications formats as any other TIPS technical area.

General information about a campus may be included as TIPS "Information Sheets." Information regarding the staff at a given campus may be captured using TIPS resume functions. Additional tables will be needed to identify each campus, provide a general welcome message, and maintain graphic files. Campus-linking tables will identify which technical domains operate under the purview of the campus. A given technical domain may be shared across physical locations as needed.

The two questions regarding users in multiple technical domains and the creation of campus-specific web sites from TIPS data will be the issues driving TIPS Phase III. A number of other issues will also be addressed during Phase III. These issues are described in the foll owing paragraphs.

Potential tie-ins between TIPS and standard Corps data sources may be prototyped during TIPS Phase III. One of these tie-ins is, for example, the link between the Corps e-mail directory (X.500) and TIPS. The Corps e-mail directory is the official directory of all Corps users and should be used as the basis for updating TIPS personnel accounts. Using the Corps e-mail directory, all TIPS users' account information may be updated automatically without the user needing to change their telephone number, address, or office affiliation. As appropriate, other interfaces between TIPS and standard Corps data sources will be identified and documented during Phase III. The interface between TIPS and the Corps of Engineers Project Management System (PROMIS), for example, will be evaluated during the ERDC-wide review of TIPS.

As more people begin to use TIPS, more organization will need to be given to technical support activities. An extension to the electronic bulletin board is planned to enable users to directly request system enhancements. A 
Configuration Control Board (CCB) of TIPS users and ERDC managers may be formed in FY00 to assist in prioritizing upgrades to TIPS.

The use of the web as an effective tool for business processing was proven in Phase I with the development of the on-line fact sheets and creation of the LCTIP. Under Phase III the on-line submission of additional publication types will be included in TIPS. This submission will route the draft documents to technical management for review and, when appropriate, to technical editors for updates. Approvals will be returned by automated e-mail and citations will be updated, as appropriate by the user or technical editing staff, when the publication is produced.

The last items to be considered prior to beginning the next phase of TIPS, but by no means the least important, is that of system documentation. Current TIPS documentation related to the Corps LCMIS requirements were prepared during TIPS Phase II. Before work can continue beyond Phase III, additional resources will be required to prepare life-cycle cost/benefits statements and update LCMIS documentation. 


\section{Conclusions}

TIPS users are realizing important benefits even during these preliminary phases of the project. First is the capability for external and internal users to access their information from anywhere in the world. With their personal passwords, internal users of TIPS may update their information without being tied to their desktops. Second is the ability of researchers to have a single source of information where all of their work, and the work of others, is cataloged. Such a catalog provides a very effective marketing tool that may be referenced in e-mail, simultaneously used during teleconferences, and remotely used during site visits. The third benefit is a reduction in the volume and frequency of data calls from management. Since the majority of management-required data is contained in the catalog, specialized reports generate the majority of standard data call information.

Business applications on the World Wide Web have significant cost advantages over traditional client/server systems. The client software for web-based systems is commercially available and provided free of charge. Thus the long-term costs of maintaining client software specific to TIPS is zero - it is part of the user's basic computer setup just like other standard applications such as word processing and e-mail. Long-term costs of the server side of web-based applications are also significantly less than those of traditional client/server systems. For example, the cost of hardware and software for TIPS is about $\$ 10,000$. Other applications may also use the same web server platform. Software development and upgrades are simplified since changes to web applications are easily made by remote development teams. If designed to take advantage of the personal nature of I nternet-based transactions, user training costs may also be reduced from weeklong official courses required for traditional software applications to short drop-in or web-interactive sessions.

In addition to the short- and long-term costs of web-based software applications, there are also benefits for an organization in general. First is that the organization's information is captured and processed in a consistent fashion across all organizational elements. Standardization of common business practices is an essential management tool for measuring and improving productivity. Standardization of selected common business practices also allows customers to find that person who can help solve their specific problem anywhere in the organization. 
TIPS provides customers with "one door" to ERDC research and development assets.

The ultimate test of computer applications/technologies is user acceptance. Given the widespread nature of web browsers and the ever increasing use of the Internet, both internal user and external customer acceptance of user-aware web-based applications such as TIPS is very high.

At the beginning of the TIPS effort, researchers asked the question, "Can the World Wide Web be used to support internal business practices and create both internal and external customer data?" Based on the results of the operational test of TIPS Phase I, and the current progress with TIPS Phase II, the answer to this question is an emphatic "Yes." By placing selected business processes such as maintaining a professional biographical sketch, creating PAO Fact Sheets, and keeping a publication list on the World Wide Web, researchers, supervisors, and managers of ERDC technical domains automatically generate web resources for internal and external customers. 


\section{Appendix A: Default Cascading Style Sheet Definitions}

Currently only a single Cascading Style Sheet (CSS) is saved for a given site. A slight enhancement would allow multiple "standard" sets of fonts, sizes, colors, etc., from which individual business area administrators might sel ect.

Different browsers support different CSS implementations* so a very small subset of CSS has been used in the demonstration site. Browsers before version 3.0 did not implement any CSS functions. Older browsers will not be able to see the fully colored and rendered pages; however, they will be able to see all the text in their browser's default font settings.

The CSS definitions implemented in the demonstration site are provided below. This information may be used to reset site settings if needed. Note that the "hover" styles only function with I nternet Explorer at this time.

CSS - Page Title

font-family: Verdana;

font-style: normal;

font-size: 18pt;

color: darkgreen;

text-transform: capitalize;

\footnotetext{
* For more information, see http://www.projectcool.com/develop/references/css_style.html
} 
CSS - Instructions

font-family: Arial;

font-si ze: 10pt;

font-weight: normal;

color: seagreen;

text-indent: 1em;

CSS - InstructionsLink

color: seagreen;

text-indent: 0em;

CSS - InstructionsLinkHover

font-weight: bold;

color: red;

text-decoration: none;

CSS - MenuHead

font-family: Verdana;

font-weight: bold;

font-size: 9pt;

color: DarkBlue;

text-indent: 0em; 
CSS - Menul tem

font-weight: normal;

font-size: 9pt;

color: blue;

text-decoration: underline;

CSS - Menul temHover

font-weight: bold;

color: red;

text-decoration: none;

CSS - Info

font-family: Verdana;

font-si ze: 10pt;

font-weight: bold;

color: darkgreen;

text-indent: 1em;

CSS - InfoLink

font-si ze: 10pt;

color: SandyBrown;

text-decoration: underline; 
CSS - InfoLinkHover

font-weight: bold;

color: Brown;

text-decoration: none;

CSS - Main

font-family: Verdana;

font-size: 10pt;

color: darkgreen;

text-indent: 1em;

CSS - MainLink

color: SandyBrown;

font-si ze: 10pt;

text-decoration: underline;

CSS - MainLinkHover

font-weight: bold;

color: Brown;

text-decoration: none; 
CSS - F eature

font-family: Verdana;

font-si ze: 10pt;

font-weight: bold;

color: darkgreen;

text-indent: 1em;

CSS - F eatureLink

color: SandyBrown;

font-si ze: 10pt;

text-decoration: underline;

CSS - FeatureLinkHover

font-weight: bold;

color: Brown;

text-decoration: none; 


\section{Appendix B: TIPS Database Structure}

This appendix contains a listing of the TIPS organizational structure. Linking relationships between tables can be identified by the PKey (primary key) and FKey (foreign key) field names.

Table B1. Admin.

\begin{tabular}{|c|c|c|}
\hline Name & Type & Size \\
\hline PKeyAdmin & Number (Long) & 4 \\
\hline SpIName & Text & 250 \\
\hline SplCompany & Text & 250 \\
\hline SplCity & Text & 250 \\
\hline SplState & Text & 250 \\
\hline SplLogo & Text & 250 \\
\hline SplMessage & Memo & - \\
\hline SpIMemo & Memo & - \\
\hline SpIPageURL & Text & 255 \\
\hline MenuBackground & Text & 255 \\
\hline Newgif1 & Text & 255 \\
\hline Newgif2 & Text & 255 \\
\hline Newcaption & Text & 255 \\
\hline Hotgif1 & Text & 255 \\
\hline Hotgif2 & Text & 255 \\
\hline Hotcaption & Text & 255 \\
\hline TopMemo & Memo & - \\
\hline Feature1caption & Text & 255 \\
\hline Feature1gif & Text & 255 \\
\hline Feature1key & Number (Long) & 4 \\
\hline Feature2caption & Text & 255 \\
\hline Feature2gif & Text & 255 \\
\hline Feature2key & Number (Long) & 4 \\
\hline Feature3caption & Text & 255 \\
\hline Feature3gif & Text & 255 \\
\hline Feature3key & Number (Long) & 4 \\
\hline PageTitleStyle & Memo & - \\
\hline FtrURL & Text & 50 \\
\hline FtrName & Text & 30 \\
\hline FtrEMail & Text & 30 \\
\hline FtrPhone & Text & 50 \\
\hline SPassword & Text & 255 \\
\hline UpdatedOn & Date/Time & 8 \\
\hline UpdatedBy & Number (Long) & 4 \\
\hline SpareTag & Yes/No & 1 \\
\hline SpareText & Text & 10 \\
\hline
\end{tabular}


Table B2. Area.

\begin{tabular}{|c|c|c|}
\hline Name & Type & Size \\
\hline PKeyArea & Number (Long) & 4 \\
\hline AreaLongName & Text & 255 \\
\hline AreaShortName & Text & 50 \\
\hline AreaAbstract & Text & 255 \\
\hline Area1Header & Text & 255 \\
\hline Area1Memo & Memo & - \\
\hline Area2Header & Text & 255 \\
\hline Area2Memo & Memo & - \\
\hline Area3Header & Text & 255 \\
\hline Area3Memo & Memo & - \\
\hline Area4Header & Text & 255 \\
\hline Area4Memo & Memo & - \\
\hline Area5Header & Text & 255 \\
\hline Area5Memo & Memo & - \\
\hline AreaGif1on & Text & 255 \\
\hline AreaGif1over & Text & 255 \\
\hline AreaGif1caption & Text & 255 \\
\hline AreaGif1link & Text & 255 \\
\hline AreaGif2on & Text & 255 \\
\hline AreaGif2over & Text & 255 \\
\hline AreaGif2caption & Text & 255 \\
\hline AreaGif2link & Text & 255 \\
\hline AreaGif3on & Text & 255 \\
\hline AreaGif3over & Text & 255 \\
\hline AreaGif3caption & Text & 255 \\
\hline AreaGif3link & Text & 255 \\
\hline LastUpdate & Date/Time & 8 \\
\hline UpdateBy & Number (Long) & 4 \\
\hline ActionMemo & Memo & - \\
\hline Approved & Yes/No & 1 \\
\hline ActionBy & Number (Long) & 4 \\
\hline ActionOn & Date/Time & 8 \\
\hline SpareText & Text & 255 \\
\hline SpareTag & Yes/No & 1 \\
\hline
\end{tabular}

Table B3. Area_Developers.

\begin{tabular}{|l|l|l|}
\hline Name & Type & Size \\
\hline PKeyArea_Developers & Number (Long) & 4 \\
\hline FKeyPerson & Number (Long) & 4 \\
\hline FKeyArea & Number (Long) & 4 \\
\hline UpdatedBy & Number (Long) & 4 \\
\hline UpdatedOn & Date/Time & 8 \\
\hline SpareTag & Yes/No & 1 \\
\hline SpareText & Text & 10 \\
\hline
\end{tabular}


Table B4. Area_Products.

\begin{tabular}{|l|l|l|}
\hline Name & Type & Size \\
\hline PKeyArea_Products & Number (Long) & 4 \\
\hline FKeyProduct & Number (Long) & 4 \\
\hline FKeyArea & Number (Long) & 4 \\
\hline UpdatedBy & Number (Long) & 4 \\
\hline UpdatedOn & Date/Time & 8 \\
\hline SpareTag & Yes/No & 1 \\
\hline SpareText & Text & 10 \\
\hline
\end{tabular}

Table B5. Area_Publications.

\begin{tabular}{|l|l|l|}
\hline Name & Type & Size \\
\hline PKeyArea_Publications & Number (Long) & 4 \\
\hline FKeyPublication & Number (Long) & 4 \\
\hline FKeyArea & Number (Long) & 4 \\
\hline UpdatedBy & Number (Long) & 4 \\
\hline UpdatedOn & Date/Time & 8 \\
\hline SpareTag & Yes/No & 1 \\
\hline SpareText & Text & 10 \\
\hline
\end{tabular}

Table B6. Award_Type.

\begin{tabular}{|l|l|l|}
\hline Name & Type & Size \\
\hline PKeyAwardType & Number (Long) & 4 \\
\hline AwardType & Text & 255 \\
\hline UpdatedOn & Date/Time & 8 \\
\hline UpdatedBy & Number (Long) & 4 \\
\hline
\end{tabular}

Table B7. Comment.

\begin{tabular}{|l|l|l|}
\hline Name & Type & Size \\
\hline PKeyCom & Number (Long) & 4 \\
\hline ComPage & Text & 255 \\
\hline ComFName & Text & 255 \\
\hline ComLName & Text & 255 \\
\hline ComPhone & Text & 255 \\
\hline ComEmail & Text & 255 \\
\hline ComComment & Memo & - \\
\hline ComNeeds & Yes/No & 1 \\
\hline ComDate & Date/Time & 8 \\
\hline ComResponse & Memo & - \\
\hline ComResponseBy & Number (Long) & 4 \\
\hline ComResponseDate & Date/Time & 8 \\
\hline
\end{tabular}


Table B8. Degree_Type.

\begin{tabular}{|l|l|l|}
\hline Name & Type & Size \\
\hline PKeyDegType & Number (Long) & 4 \\
\hline DegType & Text & 255 \\
\hline UpdatedOn & Date/Time & 8 \\
\hline UpdatedBy & Number (Long) & 4 \\
\hline
\end{tabular}

Table B9. Help.

\begin{tabular}{|l|l|l|}
\hline Name & Type & Size \\
\hline PKeyHelp & Number (Long) & 4 \\
\hline HelpWebPage & Text & 255 \\
\hline HelpFName & Text & 255 \\
\hline HelpLName & Text & 255 \\
\hline HelpPhone & Text & 255 \\
\hline HelpEmail & Text & 255 \\
\hline HelpComment & Memo & - \\
\hline HelpNeeds & Yes/No & 1 \\
\hline HelpDate & Date/Time & 8 \\
\hline HelpResponse & Memo & - \\
\hline HelpResponseBy & Number (Long) & 4 \\
\hline HelpResponseDate & Date/Time & 8 \\
\hline
\end{tabular}

Table B10. HelpPage.

\begin{tabular}{|l|l|l|}
\hline Name & Type & Size \\
\hline PkeyHelp & Number (Long) & 4 \\
\hline PageName & Text & 255 \\
\hline PageOption & Number (Byte) & 1 \\
\hline HelpStyle & Number (Byte) & 1 \\
\hline P0Head & Text & 255 \\
\hline P0Memo & Memo & - \\
\hline P0Graphic & Text & 255 \\
\hline P0GraphicAlign & Number (Byte) & 1 \\
\hline P1Head & Text & 255 \\
\hline P1Memo & Memo & - \\
\hline P1Graphic & Text & 255 \\
\hline P1GraphicAlign & Number (Byte) & 1 \\
\hline P2Head & Text & 255 \\
\hline P2Memo & Memo & - \\
\hline P2Graphic & Text & 255 \\
\hline P2GraphicAlign & Number (Byte) & 1 \\
\hline P3Head & Text & 255 \\
\hline P3Memo & Memo & - \\
\hline P3Graphic & Text & 255 \\
\hline P3GraphicAlign & Number (Byte) & 1 \\
\hline P4Head & Text & 255 \\
\hline P4Memo & Memo & - \\
\hline & & \\
\hline
\end{tabular}




\begin{tabular}{|c|c|c|}
\hline Name & Type & Size \\
\hline P4Graphic & Text & 255 \\
\hline P4GraphicAlign & Number (Byte) & 1 \\
\hline P5Head & Text & 255 \\
\hline P5Memo & Memo & - \\
\hline P5Graphic & Text & 255 \\
\hline P5GraphicAlign & Number (Byte) & 1 \\
\hline P6Head & Text & 255 \\
\hline P6Memo & Memo & - \\
\hline P6Graphic & Text & 255 \\
\hline P6GraphicAlign & Number (Byte) & 1 \\
\hline P7Head & Text & 255 \\
\hline P7Memo & Memo & - \\
\hline P7Graphic & Text & 255 \\
\hline P7GraphicAlign & Number (Byte) & 1 \\
\hline P8Head & Text & 255 \\
\hline P8Memo & Memo & - \\
\hline P8Graphic & Text & 255 \\
\hline P8GraphicAlign & Number (Byte) & 1 \\
\hline P9Head & Text & 255 \\
\hline P9Memo & Memo & - \\
\hline P9Graphic & Text & 255 \\
\hline P9GraphicAlign & Number (Byte) & 1 \\
\hline UpdatedBy & Number (Long) & 4 \\
\hline UpdatedOn & Date/Time & 8 \\
\hline SpareTag & Yes/No & 1 \\
\hline SpareText & Text & 255 \\
\hline
\end{tabular}

Table B11. Job_Series.

\begin{tabular}{|l|l|l|}
\hline Name & Type & Size \\
\hline PkeyJobSeries & Number (Long) & 4 \\
\hline PositionTitle & Text & 36 \\
\hline SeriesCode & Text & 4 \\
\hline UpdatedBy & Text & 255 \\
\hline UpdatedOn & Date/Time & 8 \\
\hline
\end{tabular}


Table B12. Organizations.

\begin{tabular}{|c|c|c|}
\hline Name & Type & Size \\
\hline PkeyOrganization & Number (Long) & 4 \\
\hline FkeyParent & Number (Long) & 4 \\
\hline Level & Number (Long) & 4 \\
\hline Supervisor & Number (Long) & 4 \\
\hline AlternateSupervisor1 & Number (Long) & 4 \\
\hline AlternateSupervisor2 & Number (Long) & 4 \\
\hline AreProgramManagers & Yes/No & 1 \\
\hline AreDevelopers & Yes/No & 1 \\
\hline ArePAO & Yes/No & 1 \\
\hline OrgName & Text & 255 \\
\hline OrgSymbol & Text & 255 \\
\hline StreetAddress & Text & 255 \\
\hline BoxAddress & Text & 255 \\
\hline City & Text & 255 \\
\hline StateProvince & Text & 255 \\
\hline PostalCode & Text & 255 \\
\hline Country & Text & 255 \\
\hline URL & Text & 255 \\
\hline LastUpdate & Date/Time & 8 \\
\hline Approved & Yes/No & 1 \\
\hline ApprovedOn & Date/Time & 8 \\
\hline ApprovedBy & Number (Long) & 4 \\
\hline SpareTag & Yes/No & 1 \\
\hline SpareText & Text & 10 \\
\hline Password & Text & 255 \\
\hline
\end{tabular}


Table B13. Personnel.

\begin{tabular}{|c|c|c|}
\hline Name & Type & Size \\
\hline PKeyPerson & Number (Long) & 4 \\
\hline FKeyOrganization & Number (Long) & 4 \\
\hline FKeySupervisor & Number (Long) & 4 \\
\hline FKeyJobSeries & Number (Long) & 4 \\
\hline AllowBio & Yes/No & 1 \\
\hline AllowVita & Yes/No & 1 \\
\hline Prefix & Text & 4 \\
\hline FirstName & Text & 50 \\
\hline MiddleName & Text & 30 \\
\hline LastName & Text & 50 \\
\hline Suffix & Text & 10 \\
\hline JobTitle & Text & 50 \\
\hline StreetAddress & Text & 255 \\
\hline POBox & Text & 255 \\
\hline MailStop & Text & 255 \\
\hline City & Text & 255 \\
\hline State & Text & 255 \\
\hline Zip & Text & 255 \\
\hline Country & Text & 255 \\
\hline Email & Text & 250 \\
\hline WorkPhone & Text & 30 \\
\hline PhoneExt & Text & 50 \\
\hline DsnPhone & Text & 50 \\
\hline InternationalPhone & Text & 50 \\
\hline Fax & Text & 50 \\
\hline IPAddress & Text & 50 \\
\hline LastUpdate & Date/Time & 8 \\
\hline LastVisit & Date/Time & 8 \\
\hline ResumeUpdate & Date/Time & 8 \\
\hline UpdatedBy & Number (Long) & 4 \\
\hline AllowResume & Yes/No & 1 \\
\hline ApproveResume & Yes/No & 1 \\
\hline ApproveResumeBy & Number (Long) & 4 \\
\hline ApproveResumeOn & Date/Time & 8 \\
\hline AllowProducts & Yes/No & 1 \\
\hline AllowPublications & Yes/No & 1 \\
\hline AllowReports & Yes/No & 1 \\
\hline BrowserType & Text & 255 \\
\hline Photograph & Text & 50 \\
\hline Administrator & Yes/No & 1 \\
\hline PAOOfficer & Yes/No & 1 \\
\hline ProgramManager & Yes/No & 1 \\
\hline Supervisor & Yes/No & 1 \\
\hline Developer & Yes/No & 1 \\
\hline Sponsor & Yes/No & 1 \\
\hline Approved & Yes/No & 1 \\
\hline ApprovedOn & Date/Time & 8 \\
\hline ApprovedBy & Number (Long) & 4 \\
\hline SpareTag & Yes/No & 1 \\
\hline SpareText & Text & 10 \\
\hline Password & Text & 255 \\
\hline
\end{tabular}


Table B14. Personnel_Awards.

\begin{tabular}{|l|l|l|}
\hline Name & Type & Size \\
\hline PKeyAward & Number (Long) & 4 \\
\hline FKeyPerson & Number (Long) & 4 \\
\hline AwardFrom & Text & 255 \\
\hline AwardDate & Date/Time & 8 \\
\hline FKeyType & Number (Long) & 4 \\
\hline Description & Memo & - \\
\hline LastUpdate & Date/Time & 8 \\
\hline UpdatedBy & Number (Long) & 4 \\
\hline SpareTag & Yes/No & 1 \\
\hline SpareText & Text & 10 \\
\hline
\end{tabular}

Table B15. Personnel_Biography.

\begin{tabular}{|l|l|l|}
\hline Name & Type & Size \\
\hline PKeyBio & Number (Long) & 4 \\
\hline FKeyPerson & Number (Long) & 4 \\
\hline Keywords & Memo & - \\
\hline Objective & Memo & - \\
\hline DegreeOneType & Text & 50 \\
\hline DegreeOneSubject & Text & 50 \\
\hline DegreeOneSchool & Text & 50 \\
\hline DegreeOneYear & Number (Long) & 4 \\
\hline DegreeTwoType & Text & 50 \\
\hline DegreeTwoSubject & Text & 50 \\
\hline DegreeTwoSchool & Text & 50 \\
\hline DegreeTwoYear & Number (Long) & 4 \\
\hline DegreeThreeType & Text & 50 \\
\hline DegreeThreeSubject & Text & 50 \\
\hline DegreeThreeSchool & Text & 50 \\
\hline DegreeThreeYear & Number (Long) & 4 \\
\hline DegreeFourType & Text & 50 \\
\hline DegreeFourSubject & Text & 50 \\
\hline DegreeFourSchool & Text & 50 \\
\hline DegreeFourYear & Number (Long) & 4 \\
\hline DegreeFiveType & Text & 50 \\
\hline DegreeFiveSubject & Text & 50 \\
\hline DegreeFiveSchool & Text & 50 \\
\hline DegreeFiveYear & Number (Long) & 4 \\
\hline LastUpdate & Date/Time & 8 \\
\hline UpdatedBy & Number (Long) & 4 \\
\hline Approved & Yes/No & 1 \\
\hline ApprovedOn & Date/Time & 8 \\
\hline ApprovedBy & Number (Long) & 4 \\
\hline SpareTag & Yes/No & 1 \\
\hline SpareText & Text & 10 \\
\hline & & \\
\hline & & \\
\hline & & \\
\hline & & \\
\hline
\end{tabular}


Table B16. Personnel_Degrees.

\begin{tabular}{|l|l|l|}
\hline Name & Type & Size \\
\hline PKeyDegree & Number (Long) & 4 \\
\hline FKeyPerson & Number (Long) & 4 \\
\hline FKeyType & Number (Long) & 4 \\
\hline DegreeSubject & Text & 50 \\
\hline DegreeSchool & Text & 50 \\
\hline DegreeYear & Date/Time & 8 \\
\hline LastUpdate & Date/Time & 8 \\
\hline UpdatedBy & Number (Long) & 4 \\
\hline SpareTag & Yes/No & 1 \\
\hline SpareText & Text & 10 \\
\hline
\end{tabular}

Table B17. Personnel_Jobs.

\begin{tabular}{|l|l|l|}
\hline Name & Type & Size \\
\hline PKeyJobs & Number (Long) & 4 \\
\hline FKeyPerson & Number (Long) & 4 \\
\hline JobTitle & Text & 50 \\
\hline Company & Text & 255 \\
\hline JobStart & Date/Time & 8 \\
\hline JobEnd & Date/Time & 8 \\
\hline Duties & Memo & - \\
\hline LastUpdate & Date/Time & 8 \\
\hline UpdatedBy & Number (Long) & 4 \\
\hline SpareTag & Yes/No & 1 \\
\hline SpareText & Text & 10 \\
\hline
\end{tabular}

Table B18. Personnel_Objective.

\begin{tabular}{|l|l|l|}
\hline Name & Type & Size \\
\hline PKeyObj & Number (Long) & 4 \\
\hline FKeyPerson & Number (Long) & 4 \\
\hline Keywords & Memo & - \\
\hline Objective & Memo & - \\
\hline LastUpdate & Date/Time & 8 \\
\hline UpdatedBy & Number (Long) & 4 \\
\hline SpareTag & Yes/No & 1 \\
\hline SpareText & Text & 10 \\
\hline
\end{tabular}


Table B19. Personnel_Registration.

\begin{tabular}{|l|l|l|}
\hline Name & Type & Size \\
\hline PKeyRegistration & Number (Long) & 4 \\
\hline FKeyPerson & Number (Long) & 4 \\
\hline FKeyType & Number (Long) & 4 \\
\hline Location & Text & 255 \\
\hline RegNumber & Text & 50 \\
\hline RegDate & Date/Time & 8 \\
\hline Approved & Yes/No & 1 \\
\hline ApprovedOn & Date/Time & 8 \\
\hline ApprovedBy & Number (Long) & 4 \\
\hline LastUpdate & Date/Time & 8 \\
\hline UpdatedBy & Number (Long) & 4 \\
\hline SpareTag & Yes/No & 1 \\
\hline SpareText & Text & 10 \\
\hline
\end{tabular}

Table B20. Personnel_Societies.

\begin{tabular}{|l|l|l|}
\hline Name & Type & Size \\
\hline PKeySocieties & Number (Long) & 4 \\
\hline FKeyPerson & Number (Long) & 4 \\
\hline Name & Text & 255 \\
\hline FKeyStatus & Number (Long) & 4 \\
\hline Activities & Memo & - \\
\hline StartDate & Date/Time & 8 \\
\hline ActivityLevel & Yes/No & 1 \\
\hline LastUpdate & Date/Time & 8 \\
\hline UpdatedBy & Number (Long) & 4 \\
\hline SpareTag & Yes/No & 1 \\
\hline SpareText & Text & 10 \\
\hline
\end{tabular}

Table B21. Process_Temp.

\begin{tabular}{|l|l|l|}
\hline Name & Type & Size \\
\hline PkeyTemp & Number (Long) & 4 \\
\hline FkeyProcess & Text & 255 \\
\hline FkeyPerson & Number (Long) & 4 \\
\hline FkeyDisplay & Number (Long) & 4 \\
\hline DisplayText & Text & 255 \\
\hline LastUpdate & Date/Time & 8 \\
\hline UpdatedBy & Number (Long) & 4 \\
\hline
\end{tabular}

Table B22. Product_Category.

\begin{tabular}{|l|l|l|}
\hline Name & Type & Size \\
\hline PkeyCategory & Number (Long) & 4 \\
\hline ProductCategory & Text & 50 \\
\hline
\end{tabular}


Table B23. Product_Developers.

\begin{tabular}{|l|l|l|}
\hline Name & Type & Size \\
\hline PKeyDevelopers & Number (Long) & 4 \\
\hline FKeyPerson & Number (Long) & 4 \\
\hline FKeyProduct & Number (Long) & 4 \\
\hline Approved & Yes/No & 1 \\
\hline ApprovedOn & Date/Time & 8 \\
\hline ApprovedBy & Number (Long) & 4 \\
\hline UpdatedBy & Number (Long) & 4 \\
\hline UpdatedOn & Date/Time & 8 \\
\hline SpareTag & Yes/No & 1 \\
\hline SpareText & Text & 10 \\
\hline
\end{tabular}

Table B24. Product_Links.

\begin{tabular}{|l|l|l|}
\hline Name & Type & Size \\
\hline FKeyProgram & Number (Long) & 4 \\
\hline FKeyProduct & Number (Long) & 4 \\
\hline UpdatedBy & Number (Long) & 4 \\
\hline UpdatedOn & Date/Time & 8 \\
\hline SpareTag & Yes/No & 1 \\
\hline SpareText & Text & 255 \\
\hline
\end{tabular}

Table B25. Product_Sponsors.

\begin{tabular}{|l|l|l|}
\hline Name & Type & Size \\
\hline PkeySponsors & Number (Long) & 4 \\
\hline FkeyPerson & Number (Long) & 4 \\
\hline FkeyProduct & Number (Long) & 4 \\
\hline Approved & Yes/No & 1 \\
\hline ApprovedOn & Date/Time & 8 \\
\hline ApprovedBy & Number (Long) & 4 \\
\hline UpdatedBy & Number (Long) & 4 \\
\hline UpdatedOn & Date/Time & 8 \\
\hline SpareTag & Yes/No & 1 \\
\hline SpareText & Text & 10 \\
\hline
\end{tabular}


Table B26. Product_TechTransfer.

\begin{tabular}{|l|l|l|}
\hline Name & Type & Size \\
\hline PKeyTechTransfer & Number (Long) & 4 \\
\hline FKeyPerson & Number (Long) & 4 \\
\hline FKeyProduct & Number (Long) & 4 \\
\hline Approved & Yes/No & 1 \\
\hline ApprovedOn & Date/Time & 8 \\
\hline ApprovedBy & Number (Long) & 4 \\
\hline UpdatedBy & Number (Long) & 4 \\
\hline UpdatedOn & Date/Time & 8 \\
\hline SpareTag & Yes/No & 1 \\
\hline SpareText & Text & 10 \\
\hline
\end{tabular}

Table B27. Products.

\begin{tabular}{|l|l|l|}
\hline Name & Type & Size \\
\hline PKeyProduct & Number (Long) & 4 \\
\hline FKeyProject & Number (Long) & 4 \\
\hline FKeyStatus & Number (Long) & 4 \\
\hline FKeyCategory & Number (Long) & 4 \\
\hline FKeyPerson & Number (Long) & 4 \\
\hline FKeyCERL & Text & 50 \\
\hline Hot & Yes/No & 1 \\
\hline Name & Text & 255 \\
\hline Description & Text & 255 \\
\hline OtherTitle1 & Text & 50 \\
\hline OtherInfo1 & Memo & - \\
\hline OtherComments1 & Memo & - \\
\hline OtherCommentsDate1 & Date/Time & 8 \\
\hline OtherTitle2 & Text & 50 \\
\hline Otherlnfo2 & Memo & - \\
\hline OtherComments2 & Memo & - \\
\hline OtherCommentsDate2 & Date/Time & 8 \\
\hline OtherTitle3 & Text & 50 \\
\hline Otherlnfo3 & Memo & - \\
\hline OtherComments3 & Memo & - \\
\hline OtherCommentsDate3 & Date/Time & 8 \\
\hline TechInfusionTag & Yes/No & 1 \\
\hline TechDescription & Memo & - \\
\hline TechRequirements & Memo & - \\
\hline TechTransition & Memo & - \\
\hline TechDemonstration & Memo & - \\
\hline TechTransfer & Memo & - \\
\hline TechImplementation & Memo & - \\
\hline TechSupport & Memo & - \\
\hline TechMaintenance & Memo & - \\
\hline Problem & Memo & - \\
\hline & & \\
\hline & & - \\
\hline
\end{tabular}




\begin{tabular}{|c|c|c|}
\hline Name & Type & Size \\
\hline ProblemComments & Memo & - \\
\hline ProblemCommentsDate & Date/Time & 8 \\
\hline Technology & Memo & - \\
\hline TechnologyComments & Memo & - \\
\hline TechnologyCommentsDate & Date/Time & 8 \\
\hline Benefits & Memo & - \\
\hline BenefitsComments & Memo & - \\
\hline BenefitsCommentsDate & Date/Time & 8 \\
\hline Status & Memo & - \\
\hline StatusComments & Memo & - \\
\hline StatusCommentsDate & Date/Time & 8 \\
\hline PointsOfContact & Memo & - \\
\hline PointsOfContactComments & Memo & - \\
\hline PointsOfContactCommentsDate & Date/Time & 8 \\
\hline Language & Text & 255 \\
\hline Interface & Text & 255 \\
\hline ProductStart & Date/Time & 8 \\
\hline ProductEnd & Date/Time & 8 \\
\hline URL & Text & 250 \\
\hline IsApplied & Yes/No & 1 \\
\hline IsBasic & Yes/No & 1 \\
\hline IsReimb & Yes/No & 1 \\
\hline IsTechTrans & Yes/No & 1 \\
\hline Approved & Yes/No & 1 \\
\hline ApprovedOn & Date/Time & 8 \\
\hline ApprovedBy & Number (Long) & 4 \\
\hline UpdatedBy & Number (Long) & 4 \\
\hline LastUpdate & Date/Time & 8 \\
\hline FSPicked & Yes/No & 1 \\
\hline FSApproved & Yes/No & 1 \\
\hline FSReviewMemo & Memo & - \\
\hline FSReviewOn & Date/Time & 8 \\
\hline FSReviewBy & Number (Long) & 4 \\
\hline SpareTag & Yes/No & 1 \\
\hline SpareText & Text & 10 \\
\hline
\end{tabular}


Table B28. Publication_POC.

\begin{tabular}{|l|l|l|}
\hline Name & Type & Size \\
\hline PKeyPubPOC & Number (Long) & 4 \\
\hline FKeyPublication & Number (Long) & 4 \\
\hline FKeyPerson & Number (Long) & 4 \\
\hline UpdatedOn & Date/Time & 8 \\
\hline UpdatedBy & Number (Long) & 4 \\
\hline SpareTag & Yes/No & 1 \\
\hline SpareText & Text & 255 \\
\hline
\end{tabular}

Table B29. Publication_Type.

\begin{tabular}{|l|l|l|}
\hline Name & Type & Size \\
\hline PKeyPublicationType & Number (Long) & 4 \\
\hline Type & Text & 50 \\
\hline UpdatedOn & Date/Time & 8 \\
\hline UpdatedBy & Number (Long) & 4 \\
\hline SpareTag & Yes/No & 1 \\
\hline SpareText & Text & 50 \\
\hline
\end{tabular}


Table B30. Publications.

\begin{tabular}{|c|c|c|}
\hline Name & Type & Size \\
\hline PKeyPublication & Number (Long) & 4 \\
\hline FKeyPerson & Number (Long) & 4 \\
\hline FKeyType & Number (Long) & 4 \\
\hline FKeyPublisher & Number (Long) & 4 \\
\hline Hot & Yes/No & 1 \\
\hline ApprovePublication & Yes/No & 1 \\
\hline ApproveBy & Number (Long) & 4 \\
\hline ApproveOn & Date/Time & 8 \\
\hline Authors & Text & 255 \\
\hline Title & Text & 255 \\
\hline Year & Number (Integer) & 2 \\
\hline Month & Number (Long) & 4 \\
\hline PubDate & Date/Time & 8 \\
\hline Volume & Text & 50 \\
\hline Issue & Text & 50 \\
\hline ISBN & Text & 50 \\
\hline ReportNumber & Text & 50 \\
\hline ADANumber & Text & 50 \\
\hline StartPage & Text & 50 \\
\hline EndPage & Text & 50 \\
\hline Abstract & Memo & - \\
\hline URL & Text & 255 \\
\hline FileName & Text & 255 \\
\hline FileGif & Text & 255 \\
\hline IsRefereed & Yes/No & 1 \\
\hline IsNonRef & Yes/No & 1 \\
\hline IsPresented & Yes/No & 1 \\
\hline IsLecture & Yes/No & 1 \\
\hline UpdateBy & Number (Long) & 4 \\
\hline LastUpdate & Date/Time & 8 \\
\hline SpareTag & Yes/No & 1 \\
\hline SpareText & Text & 50 \\
\hline
\end{tabular}

Table B31. Publishers.

\begin{tabular}{|l|l|l|}
\hline Name & Type & Size \\
\hline PkeyPublisher & Number (Long) & 4 \\
\hline Publisher & Text & 255 \\
\hline City & Text & 50 \\
\hline UpdatedOn & Date/Time & 8 \\
\hline UpdatedBy & Number (Long) & 4 \\
\hline SpareTag & Yes/No & 1 \\
\hline SpareText & Text & 50 \\
\hline
\end{tabular}


Table B32. Registration_Type.

\begin{tabular}{|l|l|l|}
\hline Name & Type & Size \\
\hline PkeyRegType & Number (Long) & 4 \\
\hline RegType & Text & 255 \\
\hline UpdatedOn & Date/Time & 8 \\
\hline UpdatedBy & Number (Long) & 4 \\
\hline
\end{tabular}

Table B33. SocietyStatus.

\begin{tabular}{|l|l|l|}
\hline Name & Type & Size \\
\hline PKeySocStatus & Number (Long) & 4 \\
\hline StatusType & Text & 255 \\
\hline UpdatedBy & Number (Long) & 4 \\
\hline UpdatedOn & Date/Time & 8 \\
\hline
\end{tabular}

Table B34. Style Sheet.

\begin{tabular}{|l|l|l|}
\hline Name & Type & Size \\
\hline PKeyStyle & Number (Long) & 4 \\
\hline StyleName & Text & 255 \\
\hline PageTitle & Memo & - \\
\hline Instructions & Memo & - \\
\hline InstructionsLink & Memo & - \\
\hline InstructionsLinkHover & Memo & - \\
\hline MenuHead & Memo & - \\
\hline Menultem & Memo & - \\
\hline MenultemHover & Memo & - \\
\hline Info & Memo & - \\
\hline InfoLink & Memo & - \\
\hline InfoLinkHover & Memo & - \\
\hline Main & Memo & - \\
\hline MainLink & Memo & - \\
\hline MainLinkHover & Memo & - \\
\hline Feature & Memo & - \\
\hline FeatureLink & Memo & - \\
\hline FeatureLinkHover & Memo & - \\
\hline Current & Yes/No & 1 \\
\hline UpdatedOn & Date/Time & 8 \\
\hline UpdatedBy & Number (Long) & \\
\hline & & - \\
\hline & Memo & - \\
\hline
\end{tabular}




\section{CERL DISTRIBUTION}

Chief of Engineers

ATTN: CEHEC-IM-LH (2)

ATTN: CEHEC-IM-LP (2)

ATTN: CECC-R

ATTN: CERD-L

ATTN: CERD-M

Commander ERDC, Vicksburg, MS

ATTN: Coastal and Hydraulics Lab, Vicksburg (2)

ATTN: Cold Regions Research, Hanover, NH (2)

ATTN: Environmental Lab, Vicksburg (2)

ATTN: Geotechnical Lab, Vicksburg (2)

ATTN: Information Technology Lab, Vicksburg (2)

ATTN: Structures Lab, Vicksburg (2)

ATTN: Topographic Engineering Center, Alexandria, VA (2)

Defense Tech Info Center 22304

ATTN: DTIC-O (2) 
Public reporting burden for this collection of information is estimated to average 1 hour per response, including the time for reviewing instructions, searching existing data sources, gathering and maintaining the data needed, and completing and reviewing the collection of information. Send comments regarding this burden estimate or any other aspect of this collection of Information, including suggestions for reducing this burden, to Washington

Headquarters Services, Directorate for information Operations and Reports, 1215 Jefferson Davis Highway, Suite 1204, Arlington, VA 22202-4302, and to

\begin{tabular}{|l|l|l|}
\hline $\begin{array}{l}\text { 1. AGENCY USE ONLY (Leave } \\
\text { Blank) }\end{array}$ & $\begin{array}{c}\text { 2. REPORT DATE } \\
\text { September } 1999\end{array}$ & $\begin{array}{c}\text { 3. REPORT TYPE AND DATES COVERED } \\
\text { Final }\end{array}$ \\
\hline
\end{tabular}

4. TITLE AND SUBTITLE

5. FUNDING NUMBERS

Providing Technology Information, Products, and Services (TIPS) Through the World Wide Web

6. AUTHOR(S)

E. William East, Sara E. Ort, and William D. Goran

7. PERFORMING ORGANIZATION NAME(S) AND ADDRESS(ES)

U.S. Army Construction Engineering Research Laboratory (CERL)

P.O. Box 9005

Champaign, IL 61826-9005

8. PEFORMING ORGANIZATION REPORT NUMBER

TR 99/80

9. SPONSORING / MONITORING AGENCY NAME(S) AND ADDRESS(ES)

U.S. Army Construction Engineering Research Laboratory (CERL)

10. SPONSORING / MONITORING AGENCY REPORT NUMBER

ATTN: CV-PP

P.O. Box 9005

Champaign, IL 61826-9005

9. SUPPLEMENTARY NOTES

Copies are available from the National Technical Information Service, 5385 Port Royal Road, Springfield, VA 22161

12a. DISTRIBUTION / AVAILABILITY STATEMENT

12b.DISTRIBUTION CODE

Approved for public release; distribution is unlimited.

13. ABSTRACT (Maximum 200 words)

The Technology Information, Products, and Services (TIPS) system is an effective tool for the capture and transfer of information about the CERL campus of the Engineer Research and Development Center (ERDC) assets. In TIPS these assets include people, capabilities, products, and publications.

Relevant information is captured as part of the daily business practices of ERDC researchers, supervisors, managers, and staff. Once approved, appropriate information is provided to potential internal and external ERDC customers.

This work builds upon the prototype Conservation Technology web site. Based on technical support feedback and a formal survey of users of the initial prototype, requirements for the TIPS were developed.

This report documents the system design, business processes, and database structures of TIPS. Initial requirements for implementation of TIPS at all ERDC campuses are also addressed. Draft Corps of Engineers' Life-Cycle Management Information System (LCMIS) documentation was prepared to document system requirements.

\begin{tabular}{|c|c|c|}
\hline $\begin{array}{l}\text { SUBJECT TERMS } \\
\text { Technology Information } \\
\text { Business practices } \\
\text { ERDC }\end{array}$ & ducts, and Services (TIPS) & $\begin{array}{l}\text { information management } \\
\text { technology transfer }\end{array}$ \\
\hline $\begin{array}{l}\text { 17. SECURITY CLASSIFICATION } \\
\text { OF REPORT } \\
\text { Unclassified }\end{array}$ & $\begin{array}{l}\text { 18. SECURITY CLASSIFICATION } \\
\text { OF THIS PAGE } \\
\text { Unclassified }\end{array}$ & $\begin{array}{l}\text { 19. SECURITY CLASSIFICATION } \\
\text { OF ASTRACT } \\
\text { Unclassified }\end{array}$ \\
\hline
\end{tabular}

